\title{
Rhodium-Catalyzed Carbonylation of Spiropentanes
}

\author{
Takanori Matsuda, Tomoya Tsuboi, and Masahiro Murakami*
}

Department of Synthetic Chemistry and Biological Chemistry, Kyoto University, Katsura, Kyoto 615-8510, Japan

\section{Supporting Information}

General. All reactions were carried out with standard Schlenk techniques under an argon, nitrogen or carbon monoxide atmosphere. Column chromatography was carried out on Kanto Silica Gel $60 \mathrm{~N}$ (spherical, neutral) (100-210 $\mu \mathrm{m}$ ). Preparative thin-layer chromatography was performed on silica gel $60 \mathrm{PF}_{254}$ (Merck). ${ }^{1} \mathrm{H}$ and ${ }^{13} \mathrm{C}$ NMR data were taken in $\mathrm{CDCl}_{3}$ at 300.07 $\mathrm{MHz}$ and $75.46 \mathrm{MHz}$, respectively. Proton chemical shifts were referenced to the residual proton signal of the solvent at $7.26 \mathrm{ppm}$. Carbon chemical shifts were referenced to the carbon signal of the solvent at $77.0 \mathrm{ppm}$.

Materials. (1-Methyl-2-methylenecyclopropyl)methanol, ${ }^{1}$ 2-cyclopropylidene-2-phenylethanol, ${ }^{2}$ 5-(4-tert-butylphenyl)spiro[2.3] hexane (10), ${ }^{3}$ (3-bromopropyl)triphenylphosphonium bromide $^{4}$ were prepared according to the literature. $p$-Xylene was distilled over sodium-benzophenone ketyl. All other commercially available chemical resources were used as received without further purification.

\section{Preparation of Spiropentanes 1}

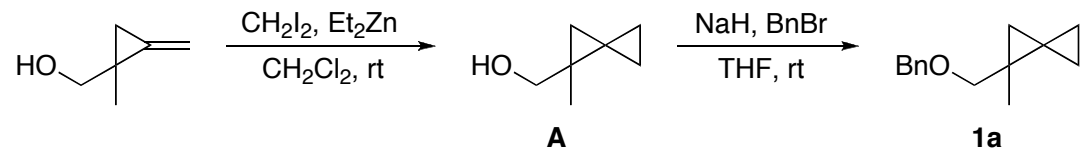

(1-Methylspiro[2.2]pent-1-yl)methanol (A). To a solution of $\mathrm{CH}_{2} \mathrm{I}_{2}(6.43 \mathrm{~g}, 24 \mathrm{mmol})$ in $\mathrm{CH}_{2} \mathrm{Cl}_{2}(70 \mathrm{~mL})$ was added dropwise a solution of $\mathrm{Et}_{2} \mathrm{Zn}$ in hexane $(1.0 \mathrm{M}, 12 \mathrm{~mL}, 12 \mathrm{mmol})$ at $-20{ }^{\circ} \mathrm{C}$. After stirring for $15 \mathrm{~min}$, a solution of (1-methyl-2-methylenecyclopropyl)methanol $(1.16 \mathrm{~g}, 11.9 \mathrm{mmol})$ in $\mathrm{CH}_{2} \mathrm{Cl}_{2}(10 \mathrm{~mL})$ was added dropwise to the reaction mixture. After being stirred overnight at room temperature, the reaction mixture was quenched with saturated $\mathrm{NH}_{4} \mathrm{Cl}$ aqueous solution, diluted with $2.0 \mathrm{~N} \mathrm{HCl}$ aqueous solution, and extracted with $\mathrm{CH}_{2} \mathrm{Cl}_{2}$. The

(1) Corlay, H.; Lewis, R. T.; Motherwell, W. B.; Shipman, M. Tetrahedron 1995, 51, 3303.

(2) Nemoto, H.; Ishibashi, H.; Nagamochi, M.; Fukumoto, K. J. Org. Chem. 1992, 57, 1707.

(3) Matsuda, T.; Shigeno, M.; Murakami, M. Chem. Lett. 2006, 35, 288.

(4) Siriwardana, A.; Nakamura, I.; Yamamoto, Y. Tetrahedron Lett. 2003, 44, 4547. 
extract was washed with saturated $\mathrm{NaHCO}_{3}$ aqueous solution, dried over $\mathrm{MgSO}_{4}$, and concentrated. The residue was purified by column chromatography on silica gel (hexane: $\mathrm{Et}_{2} \mathrm{O}=$ 2:1) to afford $\mathbf{A}(275 \mathrm{mg}, 2.46 \mathrm{mmol}, 21 \%)$.

1-(Benzyloxymethyl)-1-methylspiro[2.2]pentane (1a). To a suspension of $\mathrm{NaH}(37.2 \mathrm{mg}, 1.55$ $\mathrm{mmol})$ in THF (10 mL) was added dropwise a solution of $\mathbf{A}(125 \mathrm{mg}, 1.11 \mathrm{mmol})$ in THF $(5 \mathrm{~mL})$ at $0{ }^{\circ} \mathrm{C}$. After stirring for $10 \mathrm{~min}$, benzyl bromide $(263 \mathrm{mg}, 1.55 \mathrm{mmol}$ ) was added to the mixture. After being stirred overnight at room temperature, $\mathrm{NaH}(13.4 \mathrm{mg}, 0.56 \mathrm{mmol})$ and benzyl bromide (96 mg, $0.56 \mathrm{mmol}$ ) was added again. After being stirred overnight at room temperature, the reaction mixture was quenched with water and extracted with $\mathrm{Et}_{2} \mathrm{O}$. The extract was dried over $\mathrm{MgSO}_{4}$ and concentrated. The residue was purified by column chromatography on silica gel (hexane:AcOEt $=50: 1$, then 10:1) to afford 1a $(171 \mathrm{mg}, 0.847 \mathrm{mmol}, 76 \%):{ }^{1} \mathrm{H}$ NMR $\delta$ $0.63-0.81(\mathrm{~m}, 6 \mathrm{H}), 1.18(\mathrm{~s}, 3 \mathrm{H}), 3.32(\mathrm{~d}, J=9.6 \mathrm{~Hz}, 1 \mathrm{H}), 3.38(\mathrm{~d}, J=9.6 \mathrm{~Hz}, 1 \mathrm{H}), 4.46(\mathrm{~d}, J=$ $12.2 \mathrm{~Hz}, 1 \mathrm{H}), 4.54(\mathrm{~d}, J=12.2 \mathrm{~Hz}, 1 \mathrm{H}), 7.26-7.35(\mathrm{~m}, 5 \mathrm{H}) ;{ }^{13} \mathrm{C} \mathrm{NMR} \delta 3.5,4.6,18.2,19.2,19.6$, 19.8, 72.7, 77.6, 127.4, 127.5, 128.2, 138.8; HRMS (CI) calcd for $\mathrm{C}_{14} \mathrm{H}_{17} \mathrm{O} 201.1279\left([\mathrm{M}-\mathrm{H}]^{+}\right)$, found 201.1282 .

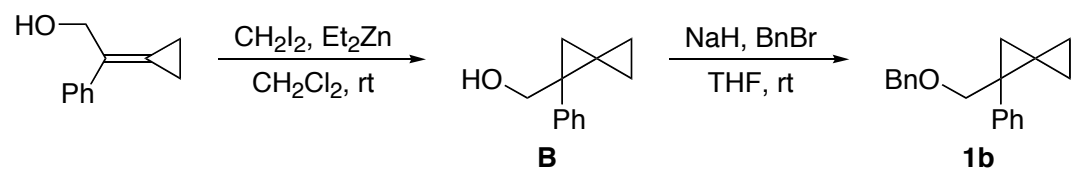

(1-Phenylspiro[2.2]pent-1-yl)methanol (B). To a solution of $\mathrm{CH}_{2} \mathrm{I}_{2}(1.37 \mathrm{~g}, 5.12 \mathrm{mmol})$ in $\mathrm{CH}_{2} \mathrm{Cl}_{2}(10 \mathrm{~mL})$ was added dropwise a solution of $\mathrm{Et}_{2} \mathrm{Zn}$ in hexane $(1.0 \mathrm{M}, 2.56 \mathrm{~mL}, 2.56 \mathrm{mmol})$ at $-20{ }^{\circ} \mathrm{C}$ and then stirred $15 \mathrm{~min}$. To the reaction mixture was added dropwise a solution of cyclopropylidene-2-phenyl-1-ethanol (205 mg, $1.28 \mathrm{mmol})$ in $\mathrm{CH}_{2} \mathrm{Cl}_{2}(10 \mathrm{~mL})$. After being stirred overnight at room temperature, the reaction mixture was quenched with saturated $\mathrm{NH}_{4} \mathrm{Cl}$ aqueous solution, diluted with $2.0 \mathrm{~N} \mathrm{HCl}$ aqueous solution, and extracted with $\mathrm{CH}_{2} \mathrm{Cl}_{2}$. The extract was washed with saturated $\mathrm{NaHCO}_{3}$ aqueous solution, dried over $\mathrm{MgSO}_{4}$, and concentrated. The residue was purified by column chromatography on silica gel (hexane:AcOEt $=3: 1)$ to afford $\mathbf{B}(132 \mathrm{mg}, 0.759 \mathrm{mmol}, 59 \%)$.

1-(Benzyloxymethyl)-1-phenylspiro[2.2]pentane (1b). To a suspension of $\mathrm{NaH}$ (73.4 mg, 3.06 $\mathrm{mmol})$ in THF $(20 \mathrm{~mL})$ a solution of $\mathbf{B}(445 \mathrm{mg}, 2.55 \mathrm{mmol})$ in THF $(5 \mathrm{~mL})$ was added dropwise at $0{ }^{\circ} \mathrm{C}$. After being stirred for $10 \mathrm{~min}$, benzyl bromide $(523 \mathrm{mg}, 3.06 \mathrm{mmol})$ was added. After being stirred for $11 \mathrm{~h}$ at room temperature, the reaction was quenched with water, extracted with $\mathrm{Et}_{2} \mathrm{O}$. The organic layer was dried over $\mathrm{MgSO}_{4}$ and concentrated. The residue was purified by column chromatography on silica gel (hexane:AcOEt $=50: 1$, then 10:1) to afford $\mathbf{1 b}(466 \mathrm{mg}$, $1.76 \mathrm{mmol}, 69 \%):{ }^{1} \mathrm{H}$ NMR $\delta 0.80-0.89(\mathrm{~m}, 3 \mathrm{H}),, 0.98-1.03(\mathrm{~m}, 1 \mathrm{H}) ,1.35(\mathrm{~d}, J=4.8 \mathrm{~Hz}, 1 \mathrm{H})$, $1.36(\mathrm{~d}, J=5.1 \mathrm{~Hz}, 1 \mathrm{H}), 3.74(\mathrm{~d}, J=10.1 \mathrm{~Hz}, 1 \mathrm{H}), 3.78(\mathrm{~d}, J=10.1 \mathrm{~Hz}, 1 \mathrm{H}), 4.51(\mathrm{~d}, J=12.3$ $\mathrm{Hz}, 1 \mathrm{H}), 4.57(\mathrm{~d}, J=12.3 \mathrm{~Hz}, 1 \mathrm{H}), 7.18-7.25(\mathrm{~m}, 1 \mathrm{H}), 7.28-7.36(\mathrm{~m}, 9 \mathrm{H}) ;{ }^{13} \mathrm{C}$ NMR $\delta 5.1,5.2$, 20.9, 22.3, 28.0, 72.9, 76.4, 125.5, 127.3, 127.4, 127.6, 127.9, 128.2, 138.5, 142.8; HRMS (CI) calcd for $\mathrm{C}_{19} \mathrm{H}_{20} \mathrm{O}\left(\mathrm{M}^{+}\right)$264.1514, found 264.1516.

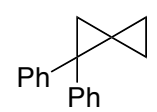

1,1-Diphenylspiro[2.2]pentane (1c). ${ }^{5} \quad{ }^{1} \mathrm{H}$ NMR $\delta$ 1.06-1.07 (m, 2H), 1.09-1.11 (m, 2H),

(5) [128742-43-8]. 
1.78-1.79 (m, 2H), 7.20-7.24 (m, 4H), 7.25-7.30 (m, 2H), 7.32-7.38 (m, 4H); ${ }^{13} \mathrm{C}$ NMR $\delta$ 7.0, 24.8, 25.0, 33.0, 125.7, 128.0, 128.4, 144.6; HRMS (CI) calcd for $\mathrm{C}_{17} \mathrm{H}_{16}\left(\mathrm{M}^{+}\right) 220.1252$, found 221.1254 .

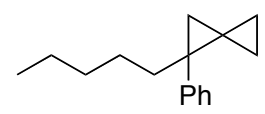

1-Pentyl-1-phenylspiro[2.2]pentane (1d). ${ }^{1} \mathrm{H}$ NMR $\delta$ 0.71-0.78 (m, 3H), 0.80-0.86 (m, 3H), 0.91-0.96 (m, $1 \mathrm{H}), 1.03(\mathrm{~d}, J=3.9 \mathrm{~Hz}, 1 \mathrm{H}), 1.02-1.36(\mathrm{~m}, 7 \mathrm{H}), 1.45-1.54(\mathrm{~m}, 1 \mathrm{H}), 1.75-1.87(\mathrm{~m}$, $1 \mathrm{H}), 7.14-7.19(\mathrm{~m}, 3 \mathrm{H}), 7.26-7.31(\mathrm{~m}, 2 \mathrm{H}) ;{ }^{13} \mathrm{C}$ NMR $\delta 5.0,5.8,14.1,20.1,22.7,22.8,26.7,28.8$, $32.2,38.2,125.4,127.9,128.0,144.6$; HRMS (CI) calcd for $\mathrm{C}_{16} \mathrm{H}_{22}\left(\mathrm{M}^{+}\right)$214.1722, found 214.1716.

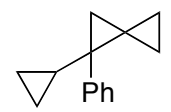

1-Cyclopropyl-1-phenylspiro[2.2]pentane (1e). ${ }^{1} \mathrm{H}$ NMR $\delta$ 0.13-0.20 (m, 1H), 0.37-0.44 (m, $1 \mathrm{H}), 0.47-0.58(\mathrm{~m}, 2 \mathrm{H}), 0.64-0.71(\mathrm{~m}, 1 \mathrm{H}), 0.74-0.80(\mathrm{~m}, 1 \mathrm{H}), 0.86-0.96(\mathrm{~m}, 2 \mathrm{H}), 1.08(\mathrm{~s}, 2 \mathrm{H})$, $1.27(\mathrm{tt}, J=8.2,5.2 \mathrm{~Hz}, 1 \mathrm{H}), 7.14-7.21(\mathrm{~m}, 1 \mathrm{H}), 7.27-7.34(\mathrm{~m}, 2 \mathrm{H}), 7.35-7.41(\mathrm{~m}, 2 \mathrm{H}) ;{ }^{13} \mathrm{C} \mathrm{NMR}$ $\delta 2.8,2.9,4.9,5.9,15.6,19.5,21.8,28.1,125.2,126.9,127.8,145.7$; HRMS (CI) calcd for $\mathrm{C}_{14} \mathrm{H}_{16}\left(\mathrm{M}^{+}\right)$184.1252, found 185.1248.

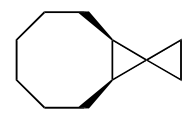

(1RS,8SR)-Spiro[bicyclo[6.1.0]nonane-9,1'-cyclopropane] (1f). ${ }^{6}{ }^{1} \mathrm{H}$ NMR $\delta 0.46-0.53(\mathrm{~m}, 2 \mathrm{H})$, 0.66-0.73 (m, 2H), 0.87-1.14 (m, 4H), 1.30-1.62 (m, 6H), 1.63-1.77 (m, 4H); ${ }^{13} \mathrm{C}$ NMR $\delta 1.1,6.2$, 20.4, 25.1, 26.7, 29.4; HRMS (CI) calcd for $\mathrm{C}_{11} \mathrm{H}_{19}\left([\mathrm{M}+\mathrm{H}]^{+}\right)$151.1487, found 151.1489.<smiles>C1CCC2CC2C2CC2C1</smiles>

$(\overline{R S}, 8 R S)-S p i r o\left[b i c y c l o[6.1 .0]\right.$ nonane-9,1'-cyclopropane] (1g). ${ }^{1} \mathrm{H}$ NMR $\delta$ 0.52-0.60 (m, 2H), 0.65-0.73 (m, 4H), 0.81-0.95 (m, 2H), 1.24-1.36 (m, 2H), 1.42-1.56 (m, 2H), 1.84-2.05 (m, 6H); ${ }^{13} \mathrm{C}$ NMR $\delta$ 3.6, 16.8, 25.8, 28.8, 31.9 [overlapping]; HRMS (FAB) calcd for $\mathrm{C}_{11} \mathrm{H}_{19}\left(\mathrm{M}^{+}\right)$ 150.1409 , found 150.1411 .

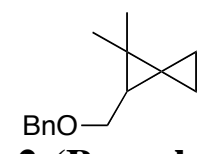

2-(Benzyloxymethyl)-1,1-dimethylspiro[2.2]pentane (1h). ${ }^{1} \mathrm{H}$ NMR $\delta$ 0.51-0.75 (m, 4H), 1.03 $(\mathrm{s}, 3 \mathrm{H}), 1.11(\mathrm{~s}, 3 \mathrm{H}), 1.14(\mathrm{t}, J=7.2 \mathrm{~Hz}, 1 \mathrm{H}), 3.47(\mathrm{dd}, J=10.3,7.7 \mathrm{~Hz}, 1 \mathrm{H}), 3.51(\mathrm{dd}, J=10.3$, $6.6 \mathrm{~Hz}, 1 \mathrm{H}), 4.50(\mathrm{~s}, 2 \mathrm{H}), 7.27-7.36(\mathrm{~m}, 5 \mathrm{H}) ;{ }^{13} \mathrm{C}$ NMR $\delta 2.3,4.3,18.2,18.9,24.5,24.9,27.1$, 70.3, 72.6, 127.4, 127.6, 128.3, 138.7; HRMS (EI) calcd for $\mathrm{C}_{15} \mathrm{H}_{20} \mathrm{O}\left(\mathrm{M}^{+}\right)$216.1514, found 216.1513 .

(6) [305370-72-3]. 


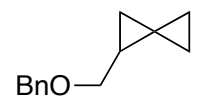

1-(Benzyloxymethyl)spiro[2.2]pentane (1i). ${ }^{1} \mathrm{H}$ NMR $\delta 0.62-0.80(\mathrm{~m}, 5 \mathrm{H}), 1.03(\mathrm{dd}, J=7.8$, $4.2 \mathrm{~Hz}, 1 \mathrm{H}), 1.46$ (ddd, $J=14.6,7.1,4.2 \mathrm{~Hz}, 1 \mathrm{H}), 3.42(\mathrm{dd}, J=12.0,6.6 \mathrm{~Hz}, 1 \mathrm{H}), 3.45$ (dd, $J=$ 12.0, 7.1 Hz, 1H), $4.48(\mathrm{~d}, J=12.2 \mathrm{~Hz}, 1 \mathrm{H}), 4.53(\mathrm{~d}, J=12.2 \mathrm{~Hz}, 1 \mathrm{H}), 7.25-7.38(\mathrm{~m}, 5 \mathrm{H}) ;{ }^{13} \mathrm{C}$ NMR $\delta 3.5,5.4,11.1,13.5,16.9,72.6,73.9,127.4,127.6,128.2,138.5$; HRMS (CI) calcd for $\mathrm{C}_{13} \mathrm{H}_{15} \mathrm{O}\left([\mathrm{M}-\mathrm{H}]^{+}\right)$187.1123, found 188.1118 .

\section{General Procedure for Rhodium-Catalyzed Carbonylation of Spiropentanes

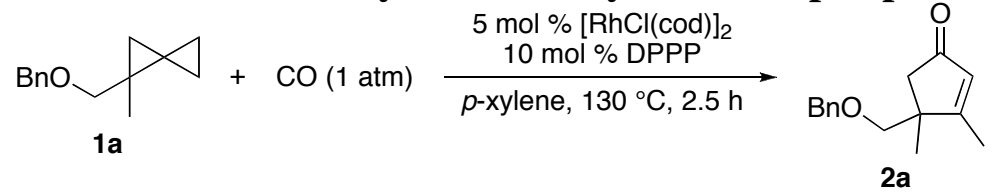

4-(Benzyloxymethyl)-3,4-dimethylcyclopent-2-enone (2a). To a mixture of $[\mathrm{RhCl}(\mathrm{cod})]_{2}$ (4.9 $\mathrm{mg}, 9.9 \mu \mathrm{mol}, 5 \mathrm{~mol} \%)$ and $\mathrm{Ph}_{2} \mathrm{P}\left(\mathrm{CH}_{2}\right)_{3} \mathrm{PPh}_{2}(8.2 \mathrm{mg}, 20 \mu \mathrm{mol}, 10 \mathrm{~mol} \%)$ under $\mathrm{CO}(1 \mathrm{~atm})$ atmosphere, $p$-xylene $(4.0 \mathrm{~mL})$ was added. The solution was stirred for $5 \mathrm{~min}$ at $130{ }^{\circ} \mathrm{C}$, then $1 \mathbf{a}$ (40.5 mg, $0.200 \mathrm{~mol}$ ) was added. After being stirred for $2.5 \mathrm{~h}$ at the same temperature, the reaction mixture was filtered through a pad of silica gel (AcOEt), and the filtrate was evaporated. The residue was purified by preparative thin-layer chromatography of silica gel (hexane:AcOEt $=2: 1)$ to afford $2 \mathrm{a}(38.7 \mathrm{mg}, 0.168 \mathrm{mmol}, 84 \%):{ }^{1} \mathrm{H} \operatorname{NMR} \delta 1.17(\mathrm{~s}, 3 \mathrm{H}), 1.99(\mathrm{~s}, 3 \mathrm{H}), 2.13(\mathrm{~d}, J$ $=18.3 \mathrm{~Hz}, 1 \mathrm{H}), 2.59(\mathrm{~d}, J=18.3 \mathrm{~Hz}, 1 \mathrm{H}), 3.33(\mathrm{~d}, J=8.9 \mathrm{~Hz}, 1 \mathrm{H}), 3.41(\mathrm{~d}, J=8.9 \mathrm{~Hz}, 1 \mathrm{H}), 4.50$ $(\mathrm{s}, 2 \mathrm{H}), 5.89(\mathrm{~s}, 1 \mathrm{H}), 7.28-7.36(\mathrm{~m}, 5 \mathrm{H}) ;{ }^{13} \mathrm{C}$ NMR $\delta$ 14.6, 22.1, 47.1, 47.4, 73.3, 74.4, 127.5, 127.7, 128.4, 131.2, 137.9, 182.0, 207.6; HRMS (FAB) calcd for $\mathrm{C}_{15} \mathrm{H}_{19} \mathrm{O}_{2}\left([\mathrm{M}+\mathrm{H}]^{+}\right)$231.1385, found 231.1389 .

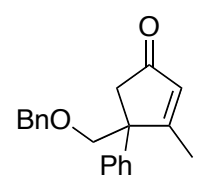

4-(Benzyloxymethyl)-3-methyl-4-phenylcyclopent-2-enone (2b). According to the general procedure, $\mathbf{2 b}$ (48.2 $\mathrm{mg}, 82 \%)$ was obtained from $\mathbf{1 b}(52.6 \mathrm{mg}, 0.199 \mathrm{mmol})$. IR (neat) 1713, $1624 \mathrm{~cm}^{-1}$; ${ }^{1} \mathrm{H}$ NMR $\delta 1.85(\mathrm{~s}, 3 \mathrm{H}), 2.51(\mathrm{~d}, J=18.6 \mathrm{~Hz}, 1 \mathrm{H}), 2.90(\mathrm{~d}, J=18.6 \mathrm{~Hz}, 1 \mathrm{H}), 3.93(\mathrm{~d}$, $J=8.7 \mathrm{~Hz}, 1 \mathrm{H}), 3.97(\mathrm{~d}, J=8.7 \mathrm{~Hz}, 1 \mathrm{H}), 4.60(\mathrm{~d}, J=12.3 \mathrm{~Hz}, 1 \mathrm{H}), 4.65(\mathrm{~d}, J=12.3 \mathrm{~Hz}, 1 \mathrm{H})$, $6.13(\mathrm{~s}, 1 \mathrm{H}), 7.13-7.15(\mathrm{~m}, 2 \mathrm{H}), 7.23-7.39(\mathrm{~m}, 8 \mathrm{H}) ;{ }^{13} \mathrm{C}$ NMR $\delta$ 15.5, 49.8, 54.8, 71.7, 73.6, 125.8, 127.0, 127.6, 127.8, 128.4, 128.8, 132.3, 137.6, 141.9, 180.5, 208.1; HRMS (FAB) calcd for $\mathrm{C}_{20} \mathrm{H}_{21} \mathrm{O}_{2}\left([\mathrm{M}+\mathrm{H}]^{+}\right)$293.1542, found 293.1536.

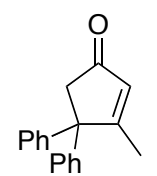

3-Methyl-4,4-diphenylcyclopent-2-enone (2c). According to the general procedure, 2c (38.8 $\mathrm{mg}, 78 \%)$ was obtained from $1 \mathrm{c}(44.2 \mathrm{mg}, 0.201 \mathrm{mmol}) .{ }^{1} \mathrm{H}$ NMR $\delta 1.94(\mathrm{~s}, 3 \mathrm{H}), 3.11(\mathrm{~s}, 2 \mathrm{H})$, $6.12(\mathrm{~s}, 1 \mathrm{H}), 7.19-7.21(\mathrm{~m}, 4 \mathrm{H}), 7.25-7.36(\mathrm{~m}, 6 \mathrm{H}) ;{ }^{13} \mathrm{C}$ NMR $\delta$ 16.9, 55.1, 60.4, 126.8, 128.0, 128.5, 131.6, 143.9, 181.8, 207.4; HRMS (EI) calcd for $\mathrm{C}_{18} \mathrm{H}_{16} \mathrm{O}\left(\mathrm{M}^{+}\right)$248.1201, found 248.1201. 


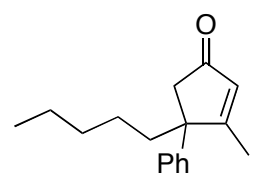

3-Methyl-4-pentyl-4-phenylcyclopent-2-enone (2d). According to the general procedure, 2d (36.8 mg, 76\%) was obtained from 1a $(42.5 \mathrm{mg}, 0.198 \mathrm{mmol}) .{ }^{1} \mathrm{H}$ NMR $\delta 0.90(\mathrm{t}, J=7.1 \mathrm{~Hz}$, $3 \mathrm{H}), 0.99-1.44(\mathrm{~m}, 6 \mathrm{H}), 1.78(\mathrm{~s}, 3 \mathrm{H}), 1.92(\mathrm{dt}, J=4.8,12.8 \mathrm{~Hz}, 1 \mathrm{H}), 2.14(\mathrm{dt}, J=3.8,12.8 \mathrm{~Hz}$, $1 \mathrm{H}), 2.55(\mathrm{~d}, J=18.8 \mathrm{~Hz}, 1 \mathrm{H}), 2.64(\mathrm{~d}, J=18.8 \mathrm{~Hz}, 1 \mathrm{H}), 6.06(\mathrm{~s}, 1 \mathrm{H}), 7.20-7.25(\mathrm{~m}, 3 \mathrm{H})$, 7.30-7.36 (m, 2H); ${ }^{13} \mathrm{C}$ NMR $\delta 14.0,15.2,22.5,23.9,32.4,34.1,50.9,53.6,125.9,126.6,128.6$, 131.5, 144.3, 182.9, 208.6; HRMS (EI) calcd for $\mathrm{C}_{17} \mathrm{H}_{22} \mathrm{O}\left(\mathrm{M}^{+}\right) 242.1671$ found 242.1676.

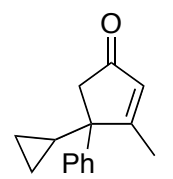

4-Cyclopropyl-3-methyl-4-phenylcyclopent-2-enone (2e). According to the general procedure, 2e $(33.8 \mathrm{mg}, 82 \%)$ was obtained from $1 \mathrm{e}(35.9 \mathrm{mg}, 0.195 \mathrm{mmol}) .{ }^{1} \mathrm{H}$ NMR $\delta 0.02-0.14(\mathrm{~m}, 1 \mathrm{H})$, 0.46-0.58 (m, 2H), 0.87-1.00 (m, 1H), 1.35-1.46 (m, 1H), $1.94(\mathrm{~d}, J=18.8 \mathrm{~Hz}, 1 \mathrm{H}), 1.94(\mathrm{~s}, 3 \mathrm{H})$, $2.11(\mathrm{~d}, J=18.8 \mathrm{~Hz}, 1 \mathrm{H}), 6.05(\mathrm{~s}, 1 \mathrm{H}), 7.22-7.29(\mathrm{~m}, 1 \mathrm{H}), 7.29-7.38(\mathrm{~m}, 2 \mathrm{H}), 7.38-7.46(\mathrm{~m}, 2 \mathrm{H})$; ${ }^{13} \mathrm{C}$ NMR $\delta-0.3,4.6,14.7,15.5,45.6,54.1,126.4,126.7,128.7,130.2,145.0,183.5,208.2$; HRMS (EI) calcd for $\mathrm{C}_{15} \mathrm{H}_{16} \mathrm{O}\left(\mathrm{M}^{+}\right)$212.1201, found 212.1191 .

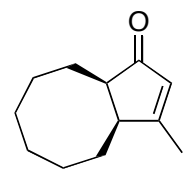

(1SR,5RS)-11-Methylbicyclo[6.3.0] undec-10-en-9-one (2f). According to the general procedure, 2f $(20.0 \mathrm{mg}, 56 \%)$ was obtained from $\mathbf{1 f}(30.1 \mathrm{mg}, 0.200 \mathrm{mmol}) .{ }^{1} \mathrm{H}$ NMR $\delta 1.24-1.85(\mathrm{~m}, 11 \mathrm{H})$, 2.07-2.14 (m, 4H), $2.29(\mathrm{t}, J=6.9 \mathrm{~Hz}, 1 \mathrm{H}), 2.72(\mathrm{t}, J=7.5 \mathrm{~Hz}, 1 \mathrm{H}), 5.88(\mathrm{~s}, 1 \mathrm{H}) ;{ }^{13} \mathrm{C} \mathrm{NMR} \delta$ $17.7,23.5,25.8,25.9,26.7,30.1,31.4,50.4,52.1,128.9,181.2,211.3$; HRMS (EI) calcd for $\mathrm{C}_{12} \mathrm{H}_{18} \mathrm{O}\left(\mathrm{M}^{+}\right)$178.1358, found 178.1363.

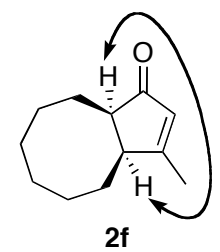

Figure 1. NOE Experiment of $\mathbf{2 f}$

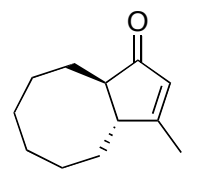

(1RS,5RS)-11-Methylbicyclo[6.3.0] undec-10-en-9-one (2g). According to the general procedure, $\mathbf{2 g}(31.3 \mathrm{mg}, 74 \%)$ was obtained from $1 \mathrm{~g}(35.9 \mathrm{mg}, 0.239 \mathrm{mmol}) .{ }^{1} \mathrm{H}$ NMR $\delta$ 1.07-1.23 (m, 2H), 1.41-1.60 (m, 4H), 1.69-1.90 (m, 4H), 1.99-2.24 (m, 6H), 2.61-2.65 (m, 2H), 5.84 (s. $1 \mathrm{H}) ;{ }^{13} \mathrm{C}$ NMR $\delta 17.2,24.7,24.8,27.0,27.3,30.3,32.1,50.0,51.9,128.9,180.6,211.05$; HRMS (EI) calcd for $\mathrm{C}_{12} \mathrm{H}_{18} \mathrm{O}\left(\mathrm{M}^{+}\right)$178.1358, found 178.1358. 


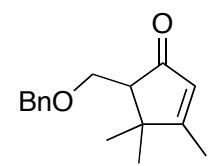

5-(Benzyloxymethyl)-3,4,4-trimethylcyclopent-2-enone (2h). According to the general procedure, $\mathbf{2 h}(34.2 \mathrm{mg}, 37 \%)$ and $\mathbf{7}(6.11 \mathrm{mg}, 18 \%)$ were obtained from $\mathbf{1 h}(34.2 \mathrm{mg}, 0.158$ mmol). 2h: ${ }^{1} \mathrm{H}$ NMR $\delta 1.18$ (s, 3H), 1.27 (s, 3H), 2.04 (s, 3H), 2.54 (dd, $\left.J=9.8,3.8 \mathrm{~Hz}, 1 \mathrm{H}\right)$, $3.60(\mathrm{t}, J=9.8 \mathrm{~Hz}, 1 \mathrm{H}), 3.89(\mathrm{dd}, J=9.8,3.8 \mathrm{~Hz}, 1 \mathrm{H}), 4.49(\mathrm{~d}, J=12.0 \mathrm{~Hz}, 1 \mathrm{H}), 4.54(\mathrm{~d}, J=$ $12.0 \mathrm{~Hz}, 1 \mathrm{H}), 5.80(\mathrm{~s}, 1 \mathrm{H}), 7.27-7.37(\mathrm{~m}, 5 \mathrm{H}) ;{ }^{13} \mathrm{C}$ NMR $\delta 14.4,22.4,26.9,46.1,58.2,67.9,73.2$, 127.5 [overlapping], 128.2, 128.3, 138.2, 185.3, 206.7; HRMS (EI) calcd for $\mathrm{C}_{16} \mathrm{H}_{20} \mathrm{O}_{2}\left(\mathrm{M}^{+}\right)$ 244.1463, found 244.1460.

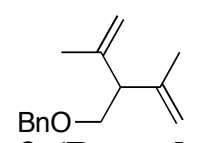

3-(Benzyloxymethyl)-2,4-dimethylpenta-1,4-diene (7). ${ }^{1} \mathrm{H}$ NMR $\delta 1.66$ (s, 6H), 2.99 (t, $J=7.4$ $\mathrm{Hz}, 1 \mathrm{H}), 3.60$ (d, $J=7.4 \mathrm{~Hz}, 2 \mathrm{H}), 4.54(\mathrm{~s}, 2 \mathrm{H}), 4.77(\mathrm{~s}, 2 \mathrm{H}), 4.88(\mathrm{~s}, 2 \mathrm{H}), 7.27-7.35(\mathrm{~m}, 5 \mathrm{H}) ;{ }^{13} \mathrm{C}$ NMR $\delta 21.0,53.5,70.3,73.1,111.8,127.5,127.7,128.3,138.4,144.0$; HRMS (EI) calcd for $\mathrm{C}_{15} \mathrm{H}_{20} \mathrm{O}\left(\mathrm{M}^{+}\right) 216.1514$, found 216.1514 .

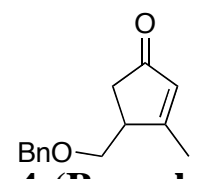

4-(Benzyloxymethyl)-3-methylcyclopent-2-enone (2i). According to the general procedure $2 \mathbf{i}$ (23.3 mg, 55\%) and $\mathbf{2}^{\prime} \mathbf{i}(8.72 \mathrm{mg}, 21 \%)$ were obtained from $\mathbf{1 i}(36.7 \mathrm{mg}, 0.195 \mathrm{mmol}) .{ }^{2} \mathbf{i}:{ }^{1} \mathrm{H}$ NMR $\delta 2.10(\mathrm{~s}, 3 \mathrm{H}), 2.31(\mathrm{dd}, J=18.4,2.3 \mathrm{~Hz}, 1 \mathrm{H}), 2.54(\mathrm{dd}, J=18.4,6.6 \mathrm{~Hz}, 1 \mathrm{H}), 3.00(\mathrm{br} \mathrm{s}$, $1 \mathrm{H}), 3.53(\mathrm{dd}, J=9.3,5.7 \mathrm{~Hz}, 1 \mathrm{H}), 3.60(\mathrm{dd}, J=9.3,4.8 \mathrm{~Hz}, 1 \mathrm{H}), 4.49(\mathrm{~d}, J=12.3 \mathrm{~Hz}, 1 \mathrm{H}), 4.54$ $(\mathrm{d}, J=12.3 \mathrm{~Hz}, 1 \mathrm{H}), 5.96(\mathrm{~s}, 1 \mathrm{H}), 7.24-7.38(\mathrm{~m}, 5 \mathrm{H}) ;{ }^{13} \mathrm{C} \mathrm{NMR} \delta 17.7,39.7,44.7,70.3,73.3$, 127.6, 127.7, 128.4, 132.0, 137.7, 178.5, 208.5; HRMS (FAB) calcd for $\mathrm{C}_{14} \mathrm{H}_{17} \mathrm{O}_{2}\left([\mathrm{M}+\mathrm{H}]^{+}\right)$ 217.1229, found 217.1228.

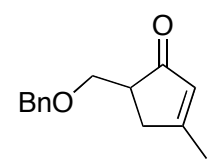

5-(Benzyloxymethyl)-3-methylcyclopent-2-enone (2'i). ${ }^{1} \mathrm{H}$ NMR $\delta 2.14(\mathrm{~s}, 3 \mathrm{H})$, 2.58-2.77 (m, $3 \mathrm{H}), 3.60-3.74(\mathrm{~m}, 2 \mathrm{H}), 4.45-4.55(\mathrm{~m}, 2 \mathrm{H}), 5.94(\mathrm{~s}, 1 \mathrm{H}), 7.24-7.38(\mathrm{~m}, 5 \mathrm{H}) ;{ }^{13} \mathrm{C}$ NMR $\delta 19.6$, 37.5, 47.4, 69.7, 73.2, 127.6 [overlapping], 128.3, 130.2, 138.1, 178.6, 209.4; HRMS (FAB) calcd for $\mathrm{C}_{14} \mathrm{H}_{17} \mathrm{O}_{2}\left([\mathrm{M}+\mathrm{H}]^{+}\right)$217.1229, found 217.1227.

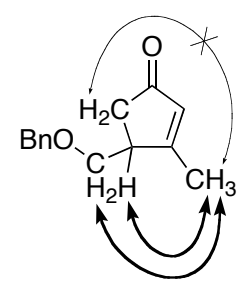

2i

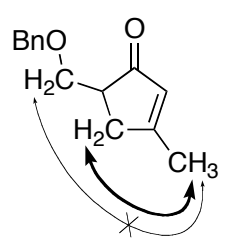

2'i

Figure 2. NOE Experiments of $\mathbf{2} \mathbf{i}$ and $\mathbf{2} \mathbf{i}$ 
$>=0$

1-(Benzyloxymethyl)spiro[2.3] hexan-5-one (8). ${ }^{1} \mathrm{H}$ NMR $\delta 0.59$ (t, $\left.J=5.2 \mathrm{~Hz}, 1 \mathrm{H}\right), 0.98$ (dd, $J$ $=8.9,5.2 \mathrm{~Hz}, 1 \mathrm{H}), 1.43(\mathrm{tt}, J=8.9,5.5 \mathrm{~Hz}, 1 \mathrm{H}), 2.91-3.27(\mathrm{~m}, 5 \mathrm{H}), 3.62(\mathrm{dd}, J=10.1,5.5 \mathrm{~Hz}$, $1 \mathrm{H}), 4.49(\mathrm{~d}, J=12.0 \mathrm{~Hz}, 1 \mathrm{H}), 4.56(\mathrm{~d}, J=12.0 \mathrm{~Hz}, 1 \mathrm{H}), 7.27-7.38(\mathrm{~m}, 5 \mathrm{H}) ;{ }^{13} \mathrm{C}$ NMR $\delta 15.2$, $15.4,20.5,50.4,54.4,72.2,72.8,127.57,127.63,128.3,138.0,206.6$; HRMS (CI) calcd for $\mathrm{C}_{14} \mathrm{H}_{16} \mathrm{O}_{2}\left(\mathrm{M}^{+}\right)$216.1150, found 216.1154.

\section{Synthesis of $( \pm)$ - $\beta$-Cuparenone (12)}
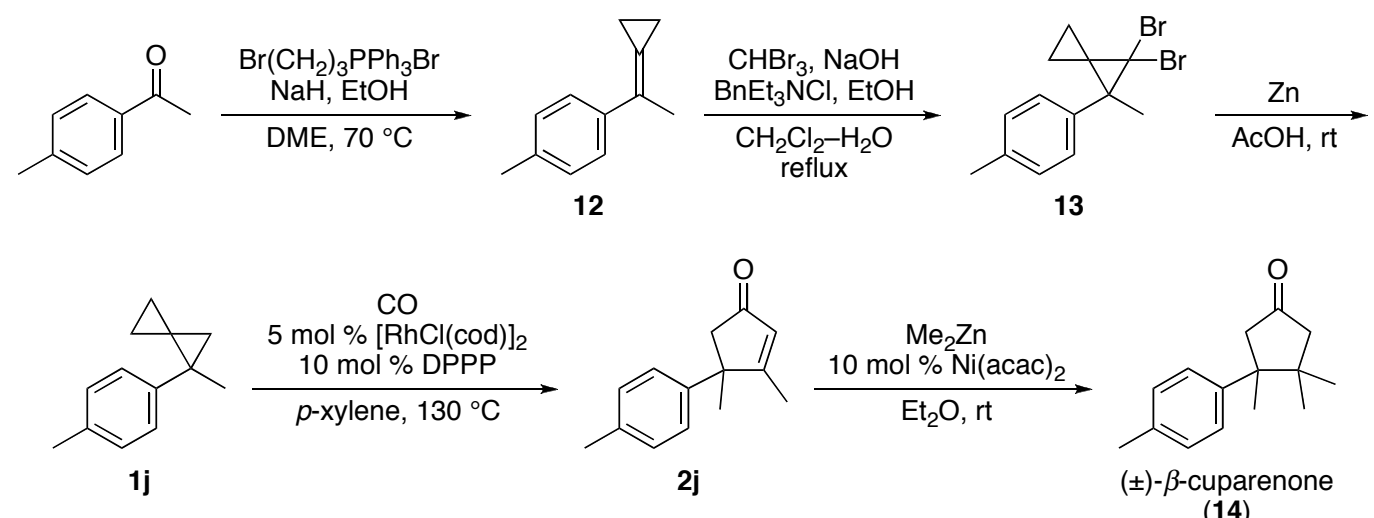

(14)

[1-(4-Methylphenyl)ethylidene]cyclopropane (12). ${ }^{7}$ To a suspension of $\mathrm{NaH}(1.03 \mathrm{~g}, 42.9$ $\mathrm{mmol}$ ) in 1,2-dimethoxyethane $(30 \mathrm{~mL})$ was added (3-bromopropyl)triphenylphosphonium bromide (10.0 g, $21.5 \mathrm{mmol})$ and $\mathrm{EtOH}$ (three drops), and the mixture was heated for $6 \mathrm{~h}$ at $60{ }^{\circ} \mathrm{C}$. To the reaction mixture was added 4'-methylacetophenone $(1.92 \mathrm{~g}, 14.3 \mathrm{mmol})$, and the mixture was heated for $20 \mathrm{~h}$ at $70{ }^{\circ} \mathrm{C}$. The reaction mixture was quenched with water and extracted with $\mathrm{Et}_{2} \mathrm{O}$. The extract was washed with brine, dried over $\mathrm{MgSO}_{4}$, and concentrated. The residue was purified by column chromatography on silica gel (hexane) to afford $\mathbf{1 2}$ (1.86 g, $11.8 \mathrm{mmol}, 83 \%)$.

1,1-Dibromo-4-methyl-4-(4-methylphenyl)spiro[2.2]pentane (13). To a solution of $\mathrm{NaOH}$ $(11.0 \mathrm{~g}, 275 \mathrm{mmol})$ in water $(11.0 \mathrm{~mL})$ was added successively $\mathrm{CHBr}_{3}(3.9 \mathrm{~mL}, 45.0 \mathrm{mmol})$, benzyltriethylammonium chloride $(42.1 \mathrm{mg}, 0.185 \mathrm{mmol})$, a solution of $12(1.61 \mathrm{~g}, 10.2 \mathrm{mmol})$ in $\mathrm{CH}_{2} \mathrm{Cl}_{2}(8 \mathrm{~mL})$, and ethanol $(220 \mu \mathrm{L})$. The mixture was refluxed for $4 \mathrm{~h}$, and then stirred at room temperature for $15 \mathrm{~h}$. The reaction mixture was quenched with water and extracted with $\mathrm{CH}_{2} \mathrm{Cl}_{2}$. The extract was washed with brine, dried over $\mathrm{MgSO}_{4}$, and concentrated. The residue was purified by column chromatography on silica gel (hexane) to afford $\mathbf{1 3}(1.81 \mathrm{~g}, 5.48 \mathrm{mmol}$, $54 \%) .{ }^{1} \mathrm{H}$ NMR $\delta 1.08-1.16(\mathrm{~m}, 1 \mathrm{H}), 1.25-1.40(\mathrm{~m}, 2 \mathrm{H}), 1.67(\mathrm{~s}, 3 \mathrm{H}), 1.73-1.81(\mathrm{~m}, 1 \mathrm{H}), 2.36(\mathrm{~s}$, $3 \mathrm{H}), 7.17(\mathrm{~d}, J=8.4 \mathrm{~Hz}, 2 \mathrm{H}), 7.23(\mathrm{~d}, J=8.4 \mathrm{~Hz}, 2 \mathrm{H}) ;{ }^{13} \mathrm{C}$ NMR $\delta 10.7,13.1,21.2,26.3,36.0$, 36.2, 48.3, 128.1, 128.7, 136.6, 139.2; HRMS (EI) calcd for $\mathrm{C}_{13} \mathrm{H}_{14} \mathrm{Br}_{2}\left(\mathrm{M}^{+}\right)$327.9462, found 327.9470 .

1-Methyl-1-(4-methylphenyl)spiro[2.2]pentane (1j). A mixture of 13 (872 mg, $2.64 \mathrm{mmol})$,

(7) [671782-19-7] 
zinc powder $(1.38 \mathrm{~g}, 21.1 \mathrm{mmol})$, and acetic acid $(15 \mathrm{~mL})$ was stirred at room temperature for 5 $\mathrm{h}$. The reaction mixture was quenched with water, filtrated $\left(\mathrm{Celite}^{\circledR}\right.$, water and $\left.\mathrm{Et}_{2} \mathrm{O}\right)$, and extracted with $\mathrm{Et}_{2} \mathrm{O}$. The extract was washed with brine, dried over $\mathrm{MgSO}_{4}$, and concentrated. The residue was purified by column chromatography on silica gel (hexane) to afford $\mathbf{1 j}$ ( $254 \mathrm{mg}$, $1.47 \mathrm{mmol}, 56 \%):{ }^{1} \mathrm{H}$ NMR $\delta$ 0.71-0.94 (m, 4H), $1.11(\mathrm{~d}, J=4.1 \mathrm{~Hz}, 1 \mathrm{H}), 1.21(\mathrm{~d}, J=4.1 \mathrm{~Hz}$, $1 \mathrm{H}), 1.44(\mathrm{~s}, 3 \mathrm{H}), 2.31(\mathrm{~s}, 3 \mathrm{H}), 7.07-7.14(\mathrm{~m}, 4 \mathrm{H}) ;{ }^{13} \mathrm{C}$ NMR $\delta 5.4,6.4,20.9,22.5,22.6,23.4$, 24.1, 125.8, 128.6, 134.4, 143.0; HRMS (EI) calcd for $\mathrm{C}_{13} \mathrm{H}_{16}\left(\mathrm{M}^{+}\right)$172.1252, found 172.1253.

3,4-Dimethyl-4-(4-methylphenyl)cyclopent-2-enone (2j). ${ }^{8}$ According to the general procedure, $2 \mathbf{j}(71.8 \mathrm{mg}, 82 \%)$ was obtained from $1 \mathbf{j}$ ( $75.3 \mathrm{mg}, 0.437 \mathrm{mmol}) .{ }^{1} \mathrm{H}$ NMR $\delta 1.63(\mathrm{~s}, 3 \mathrm{H}), 1.82$ (s, $3 \mathrm{H}), 2.32(\mathrm{~s}, 3 \mathrm{H}), 2.53(\mathrm{~d}, J=18.8 \mathrm{~Hz}, 1 \mathrm{H}), 2.65(\mathrm{~d}, J=18.8 \mathrm{~Hz}, 1 \mathrm{H}), 6.02(\mathrm{~s}, 1 \mathrm{H}), 7.08-7.16(\mathrm{~m}$, $4 \mathrm{H}) ;{ }^{13} \mathrm{C}$ NMR $\delta 14.9,20.9,23.7,49.8,54.3,125.5,129.3,130.1,136.2,141.1,184.6,208.5$; HRMS (EI) calcd for $\mathrm{C}_{14} \mathrm{H}_{16} \mathrm{O}\left(\mathrm{M}^{+}\right)$200.1201, found 200.1198.

(士)- $\boldsymbol{\beta}$-Cuparenone (14). ${ }^{9}$ To a mixture of $\mathbf{2 j}(44.9 \mathrm{mg}, 0.224 \mathrm{mmol})$ and $\mathrm{Ni}(\mathrm{acac})_{2}(5.75 \mathrm{mg}$, $22.4 \mu \mathrm{mol})$ was added dropwise a solution of $\mathrm{Me}_{2} \mathrm{Zn}$ in hexane $(1.0 \mathrm{M}, 2.5 \mathrm{~mL})$ at $0{ }^{\circ} \mathrm{C}$. After being stirred for $19 \mathrm{~h}$ at room temperature, the reaction mixture was quenched with saturated $\mathrm{NH}_{4} \mathrm{Cl}$ aqueous solution and extracted with $\mathrm{Et}_{2} \mathrm{O}$. The extract was washed with brine, dried over $\mathrm{MgSO}_{4}$, and concentrated. The residue was purified by preparative thin-layer chromatography of silica gel (hexane:AcOEt $=4: 1)$ to afford $14(36.5 \mathrm{mg}, 0.169 \mathrm{mmol}, 75 \%):{ }^{1} \mathrm{H}$ NMR $\delta 0.72(\mathrm{~s}$, $3 \mathrm{H}), 1.23(\mathrm{~s}, 3 \mathrm{H}), 1.42(\mathrm{~s}, 3 \mathrm{H}), 2.23(\mathrm{~d}, J=19.5 \mathrm{~Hz}, 1 \mathrm{H}), 2.30-2.37(\mathrm{~m}, 5 \mathrm{H}), 3.13(\mathrm{~d}, J=18.3 \mathrm{~Hz}$, $1 \mathrm{H}), 7.14(\mathrm{~d}, J=8.1 \mathrm{~Hz}, 2 \mathrm{H}), 7.20(\mathrm{~d}, J=8.4 \mathrm{~Hz}, 2 \mathrm{H}) ;{ }^{13} \mathrm{C}$ NMR $\delta 20.8,24.1,24.4,26.2,41.8$, 47.8, 50.7, 52.4, 126.5, 128.7, 135.8, 141.2, 218.3; HRMS (EI) calcd for $\mathrm{C}_{15} \mathrm{H}_{20} \mathrm{O}\left(\mathrm{M}^{+}\right)$216.1514, found 216.1511 .

(8) [133321-75-2].

(9) [25090-79-3]. 


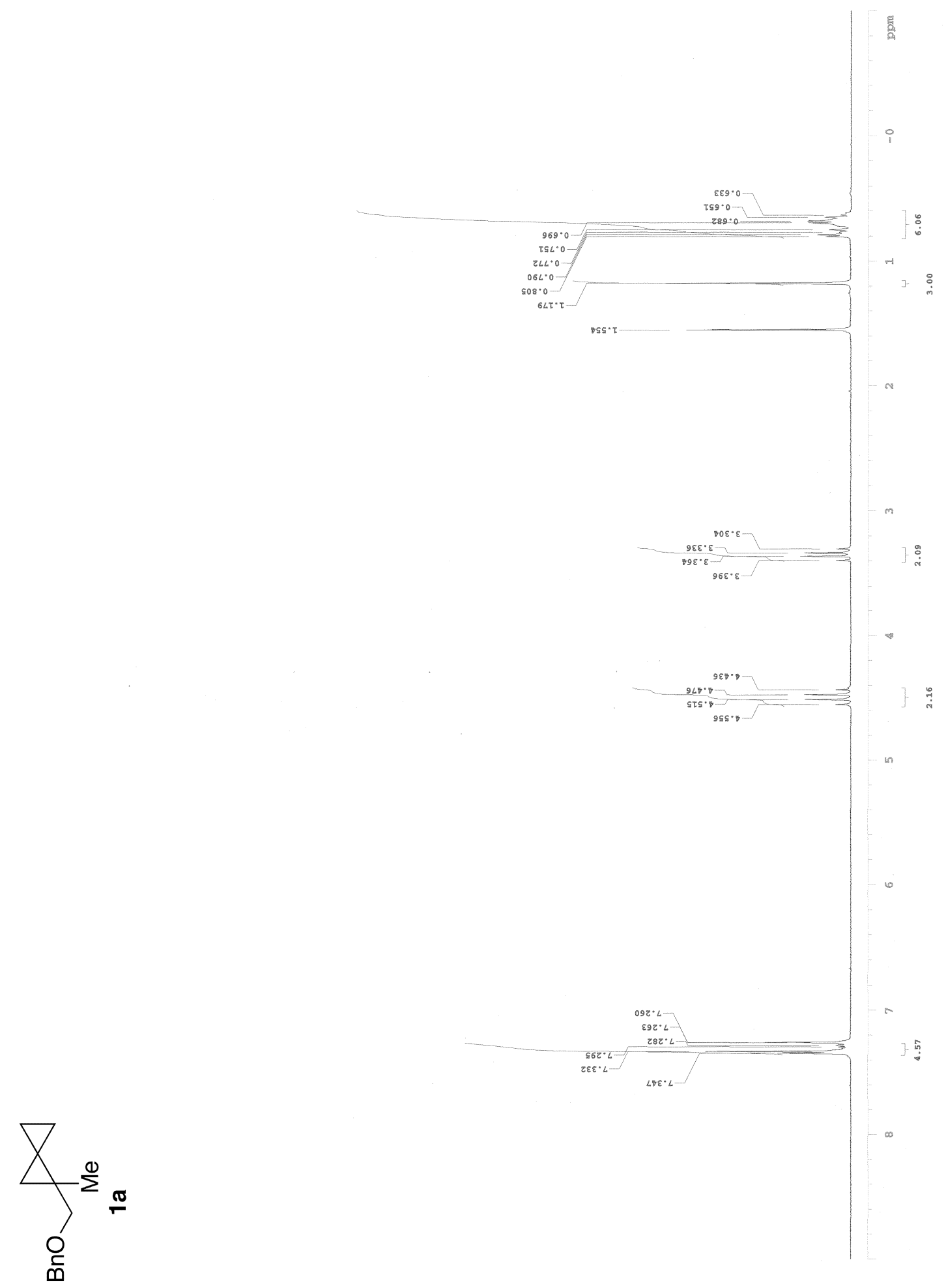




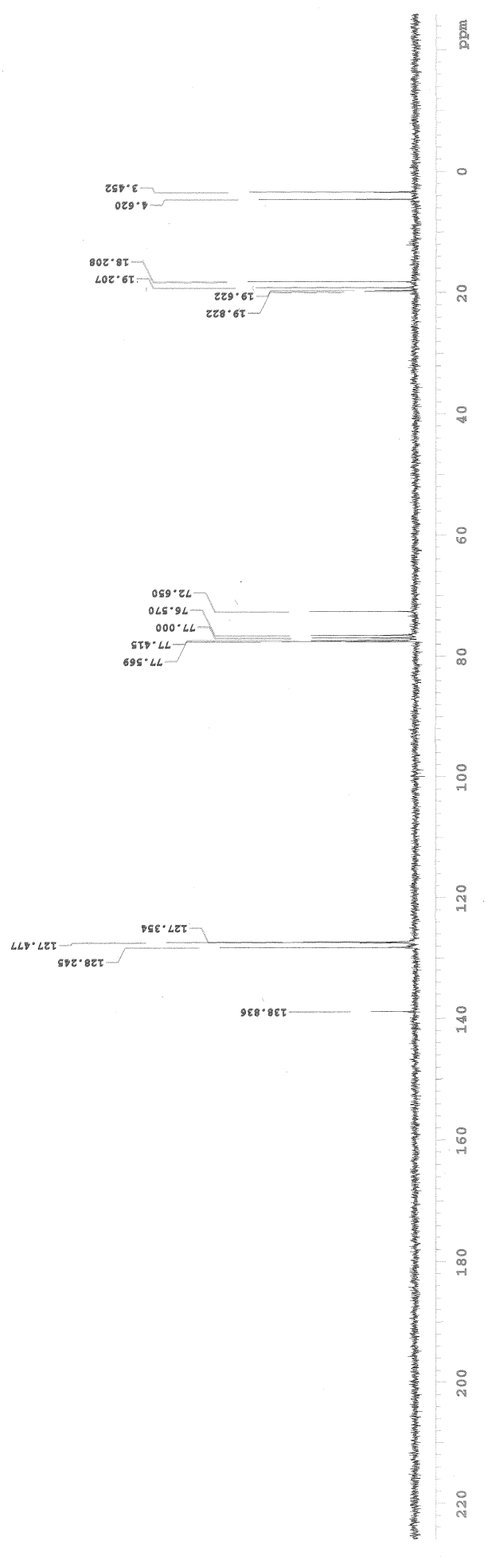




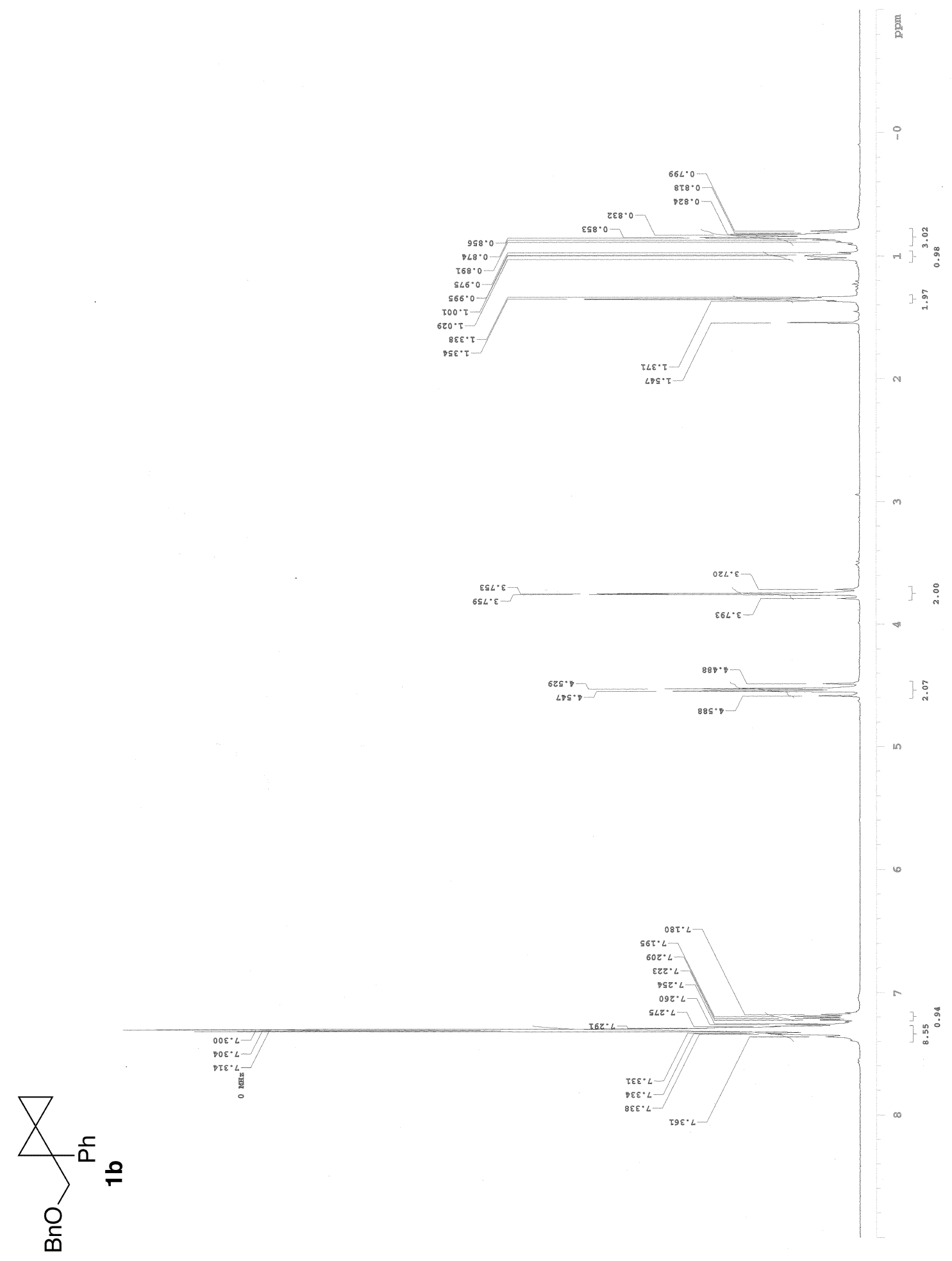




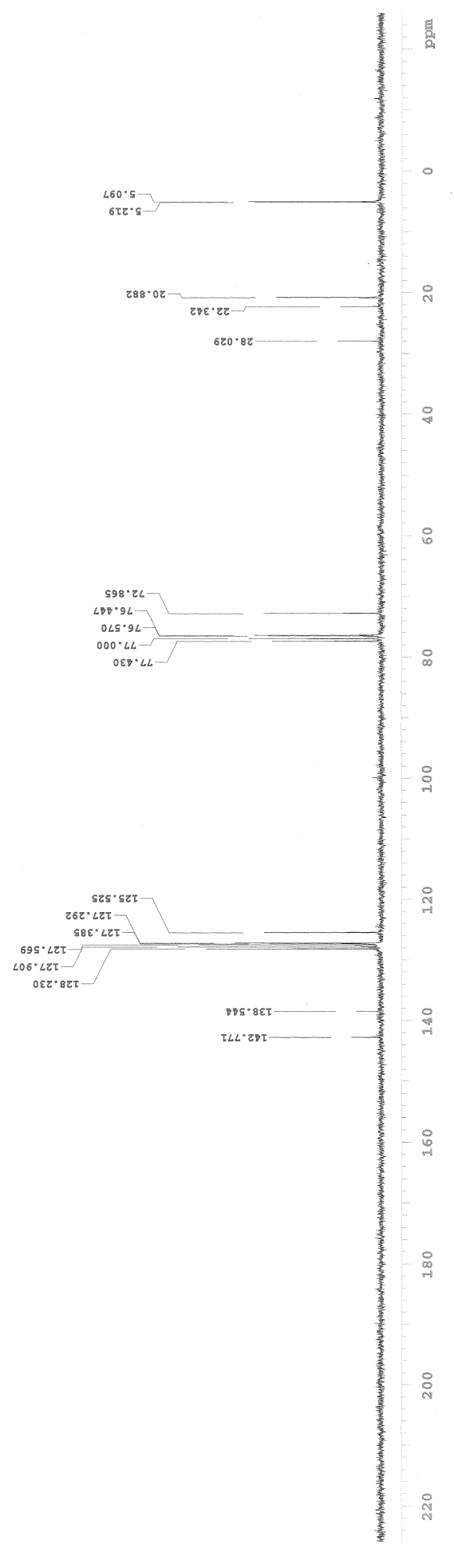




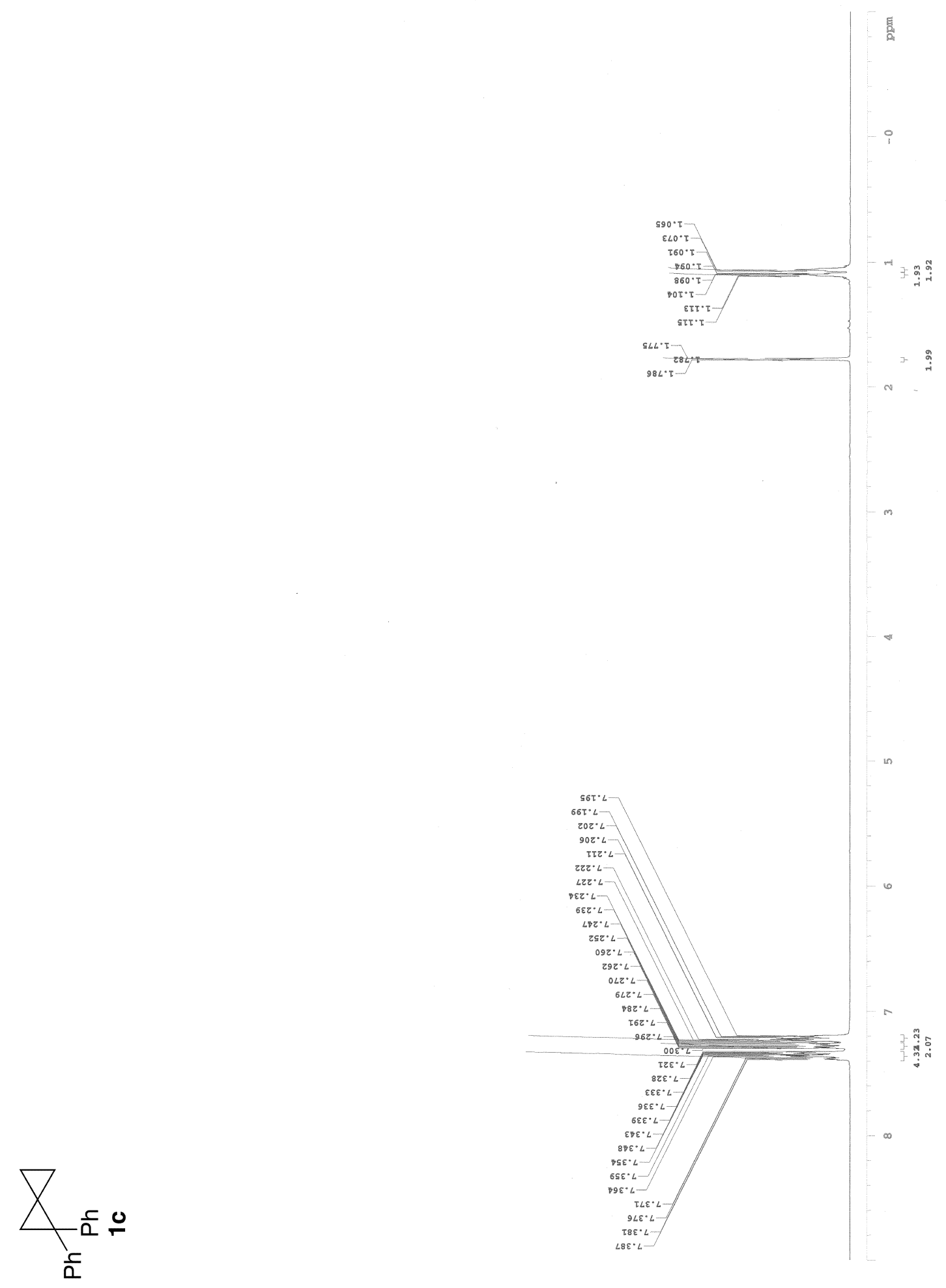




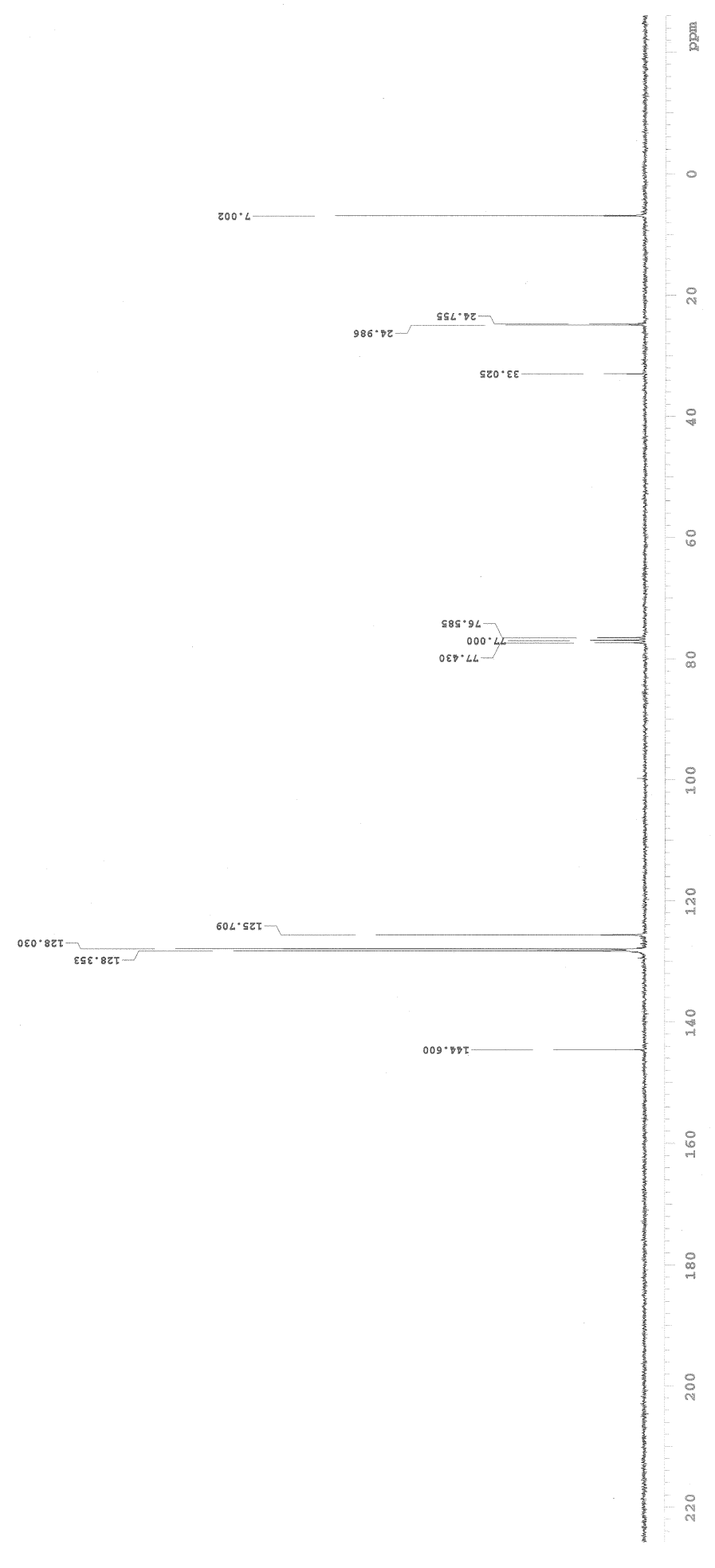



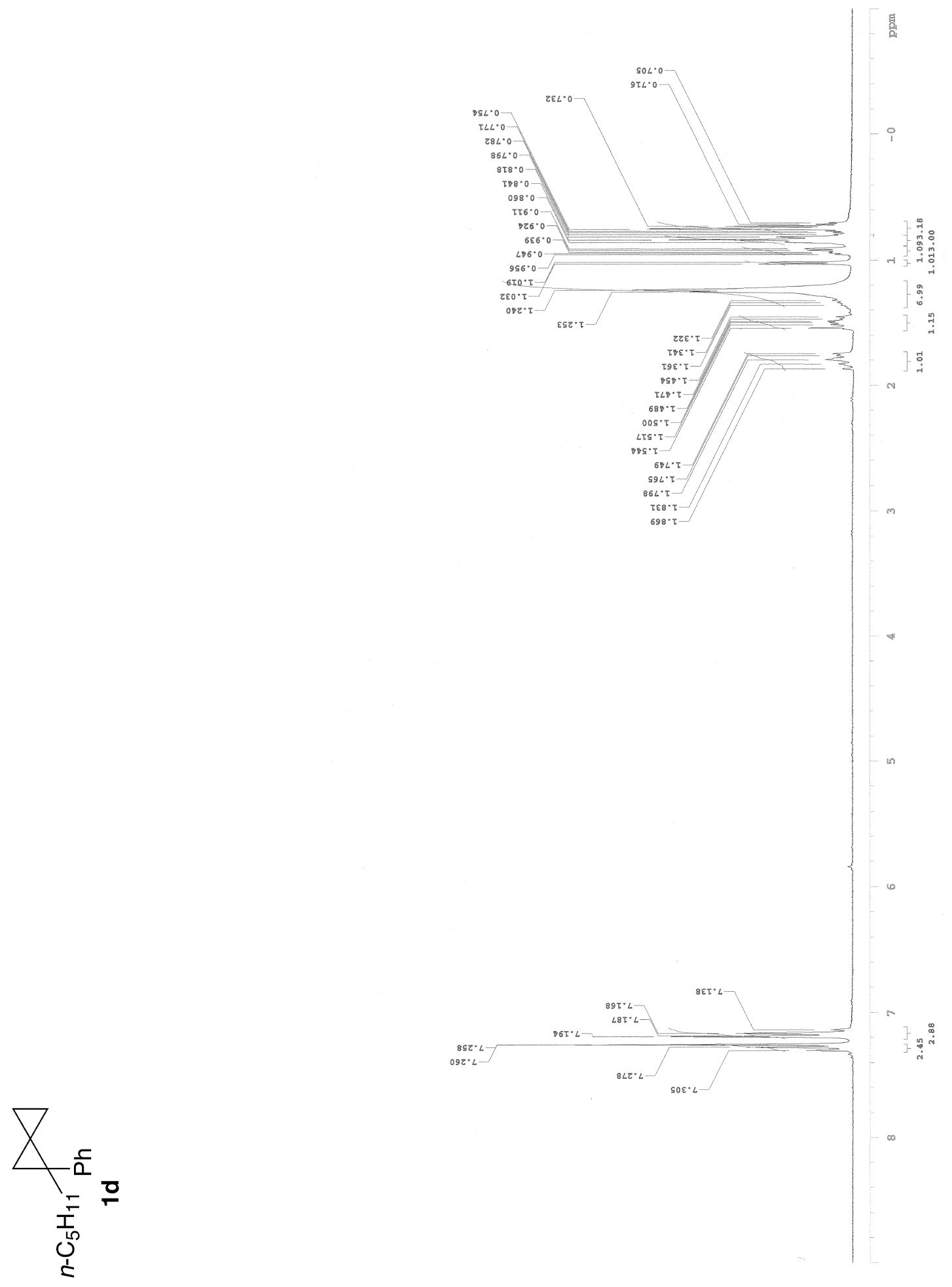


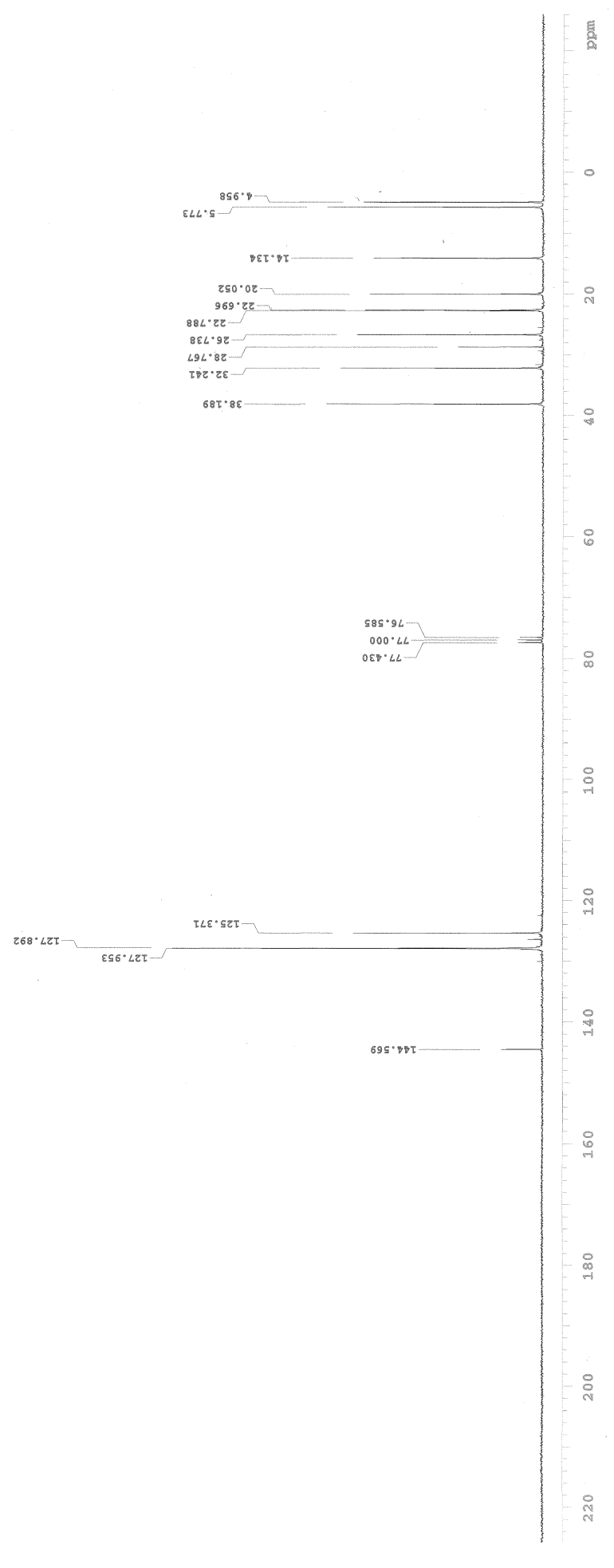




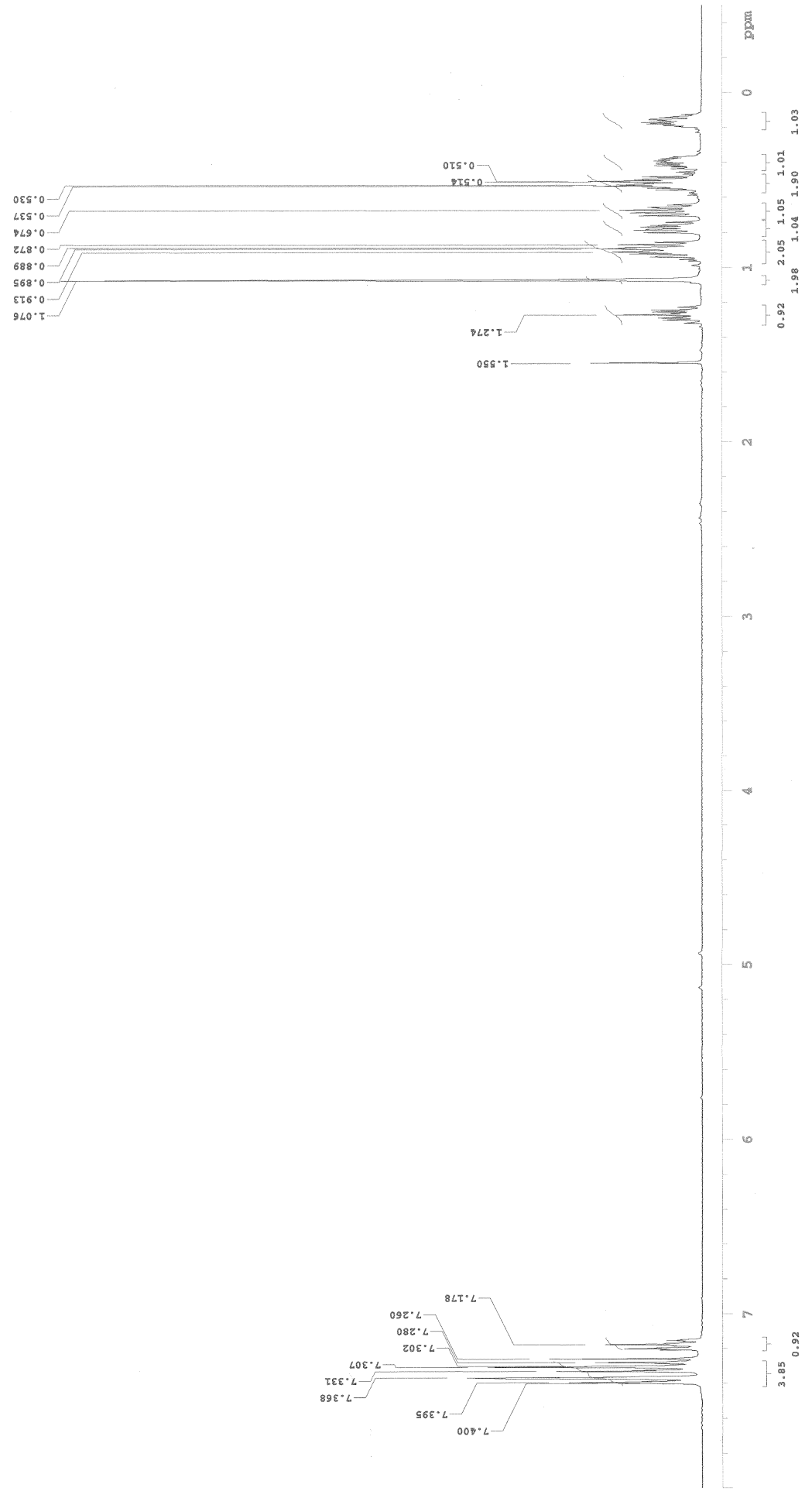




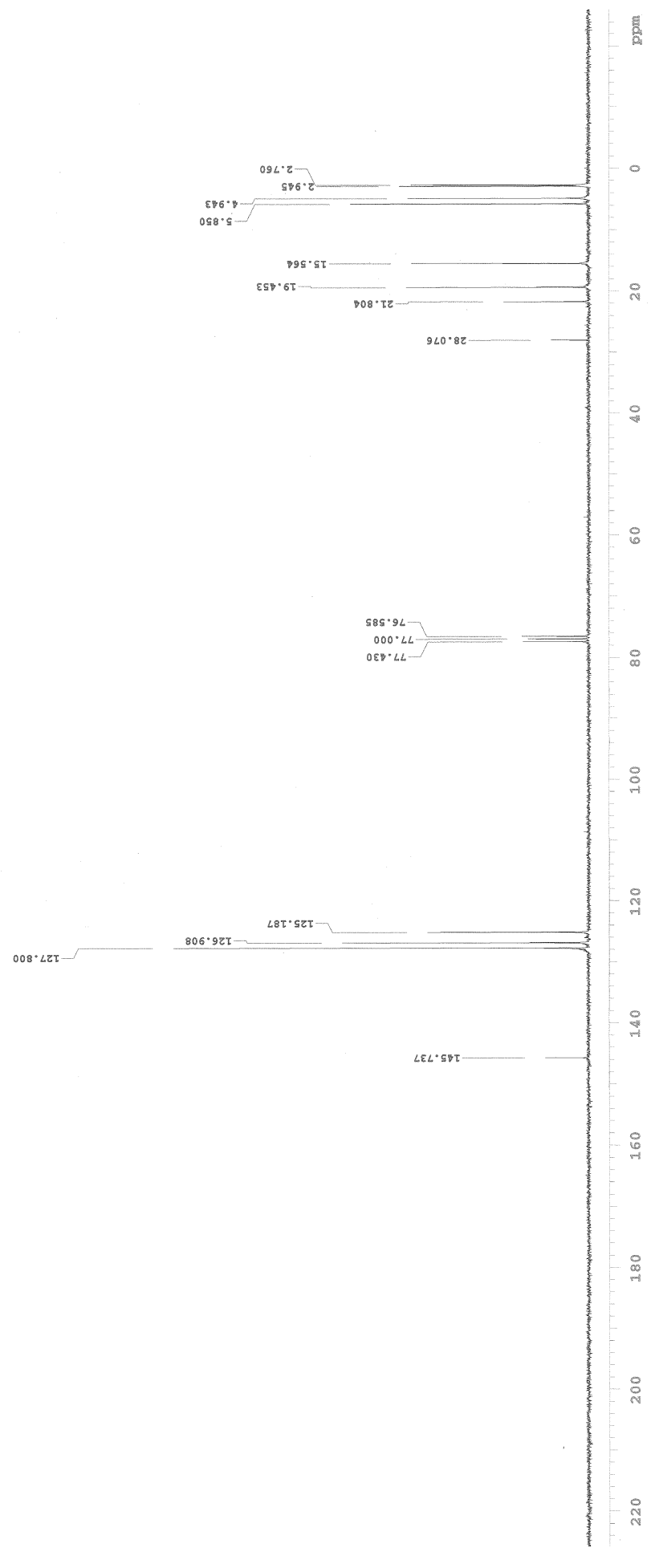




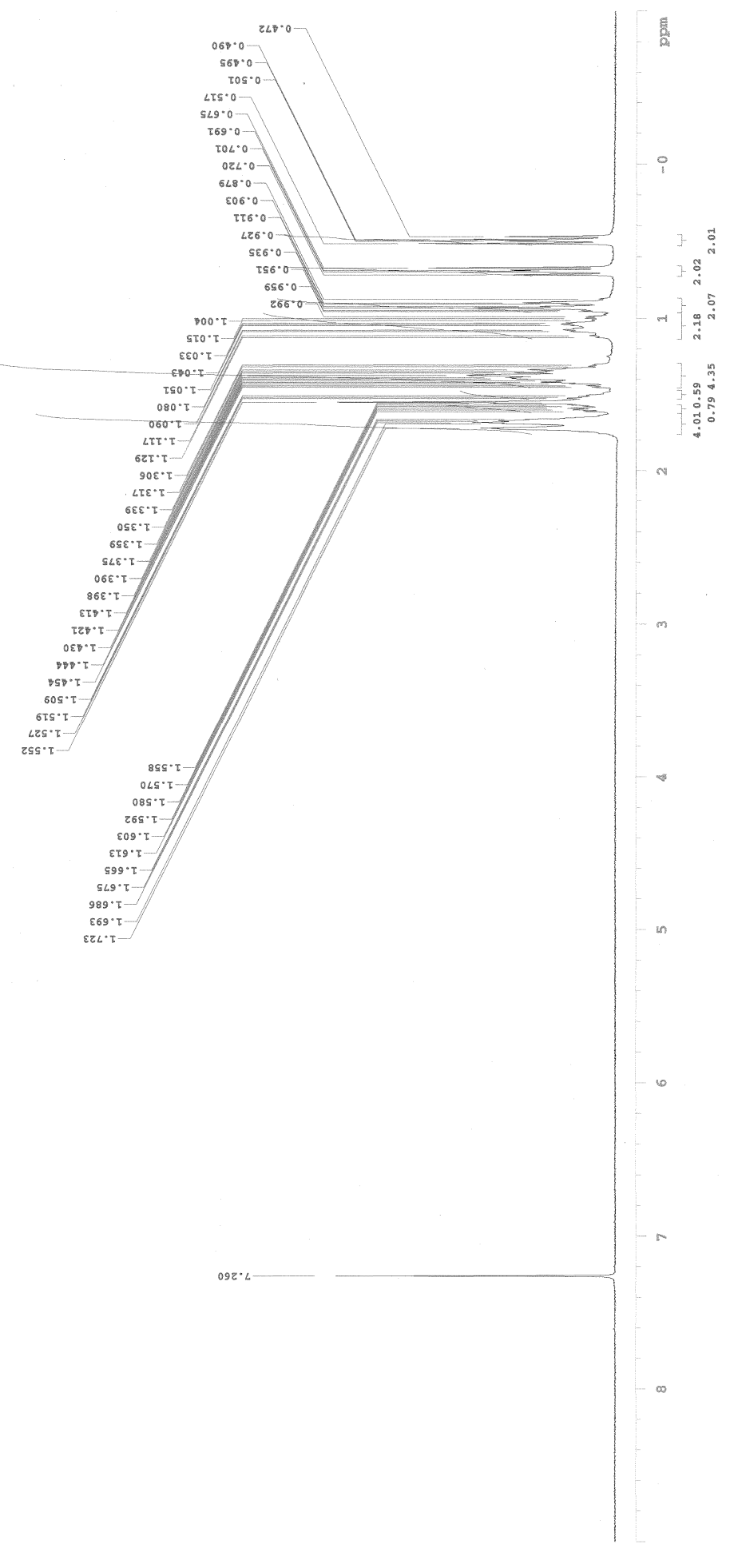




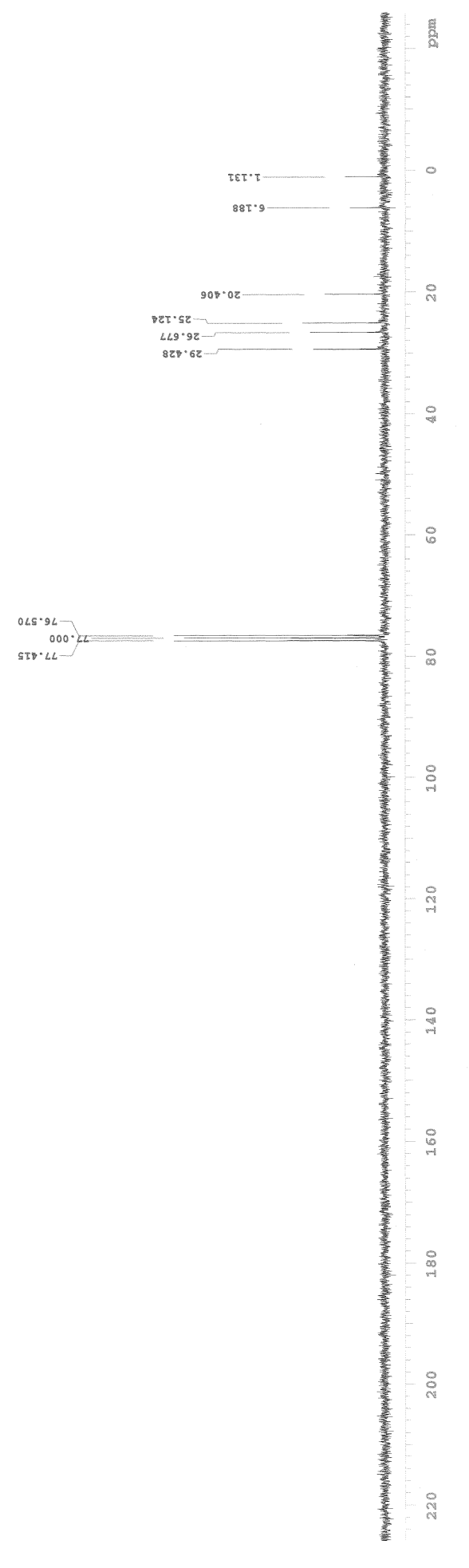




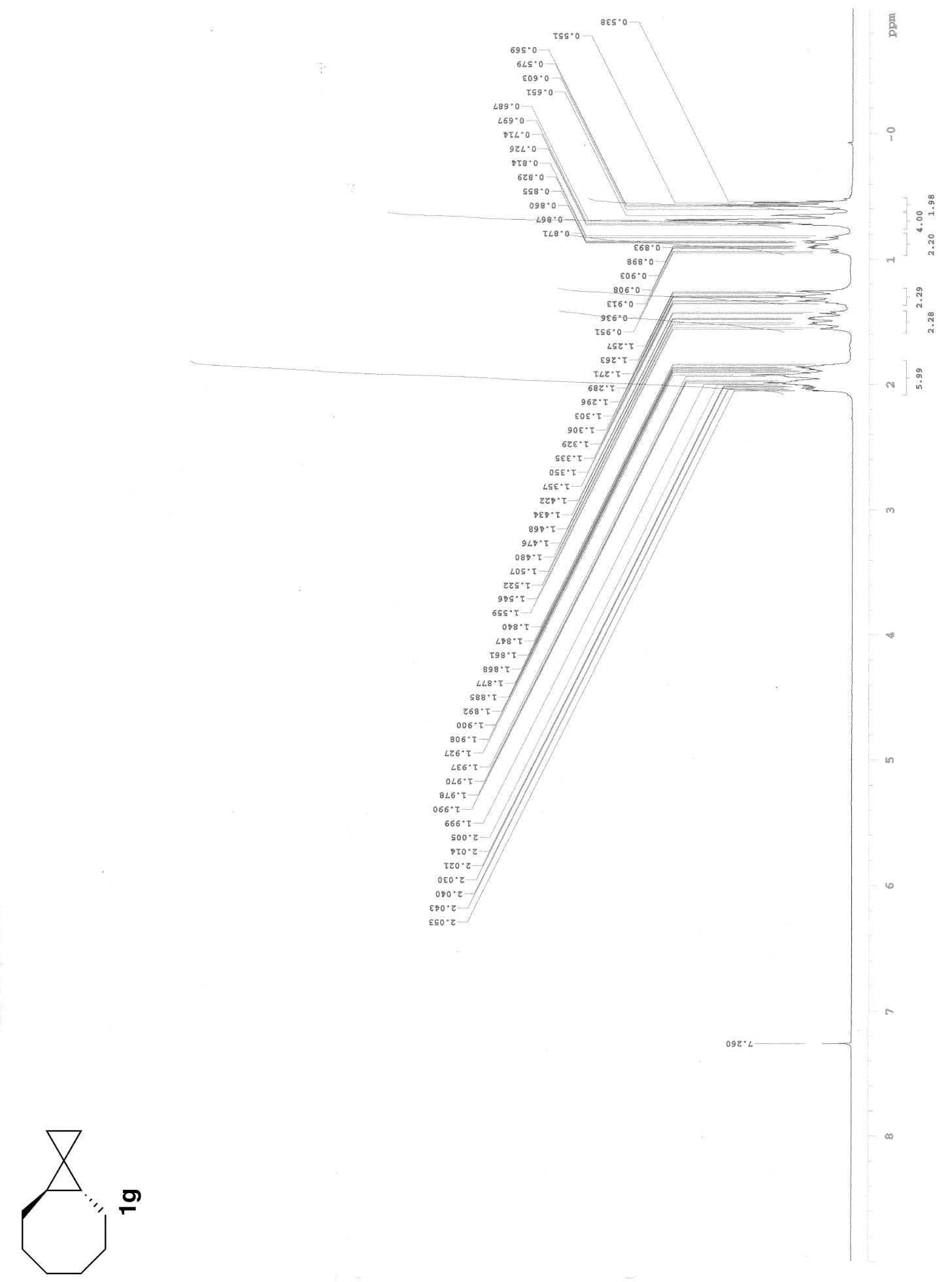




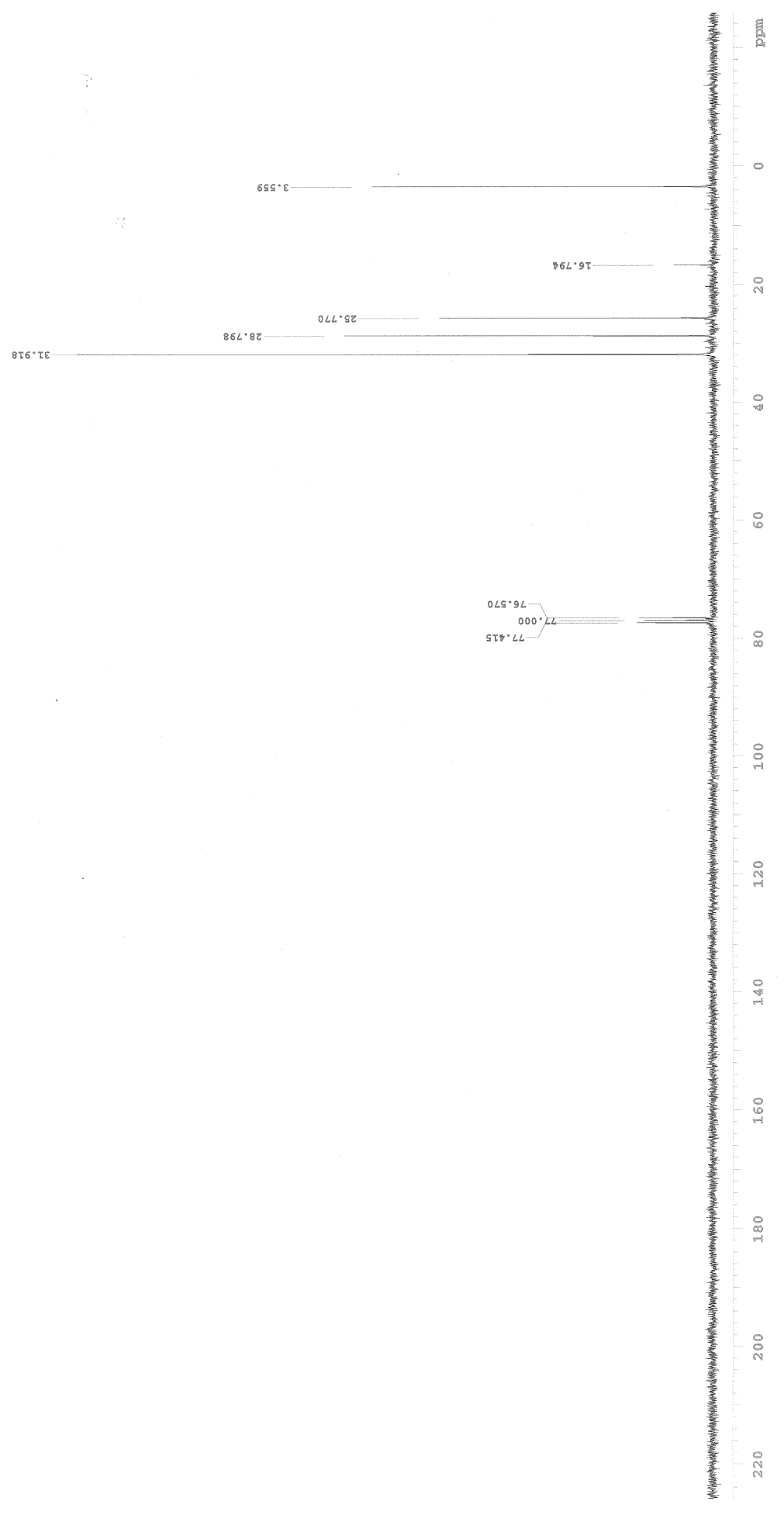




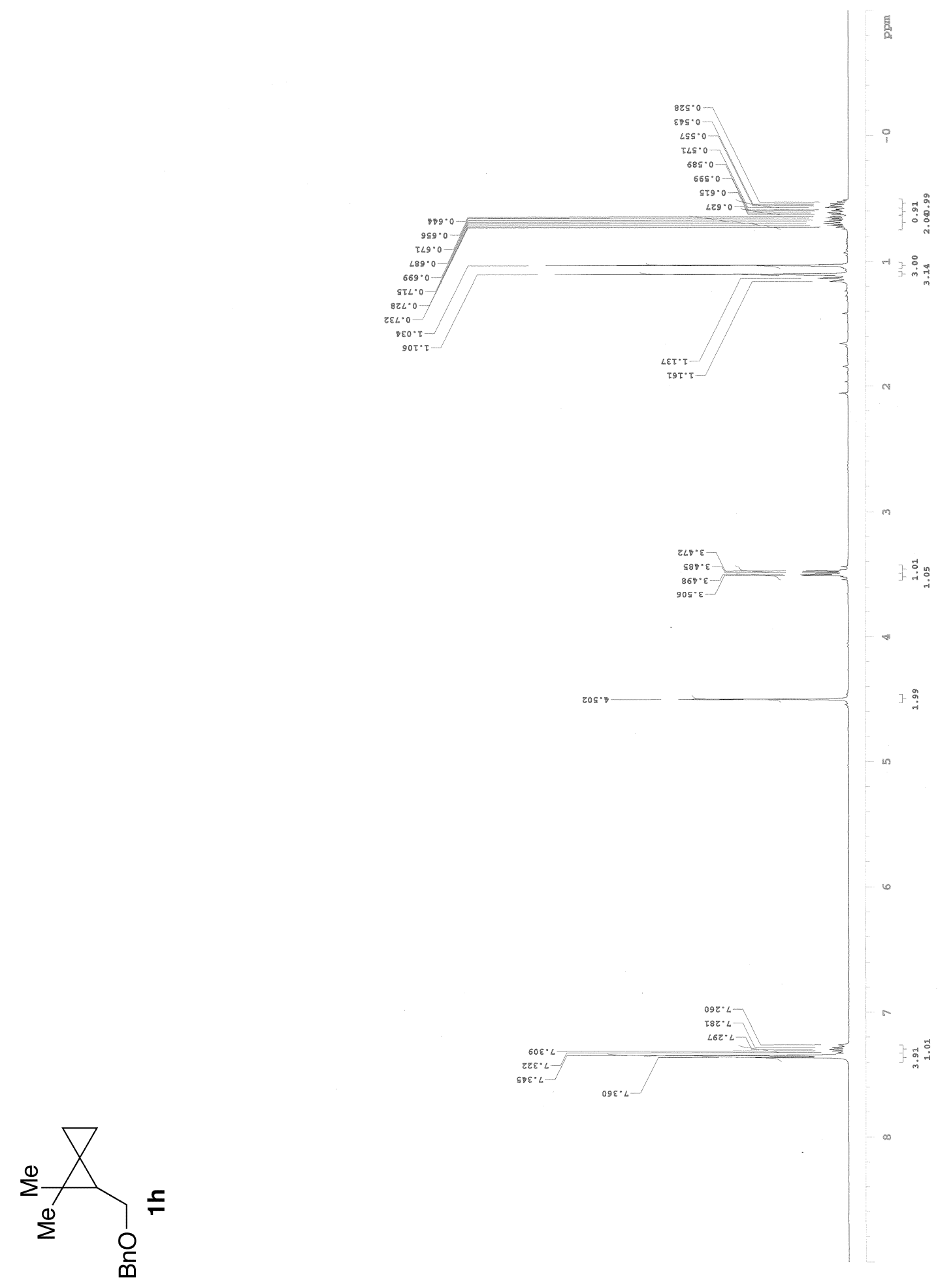




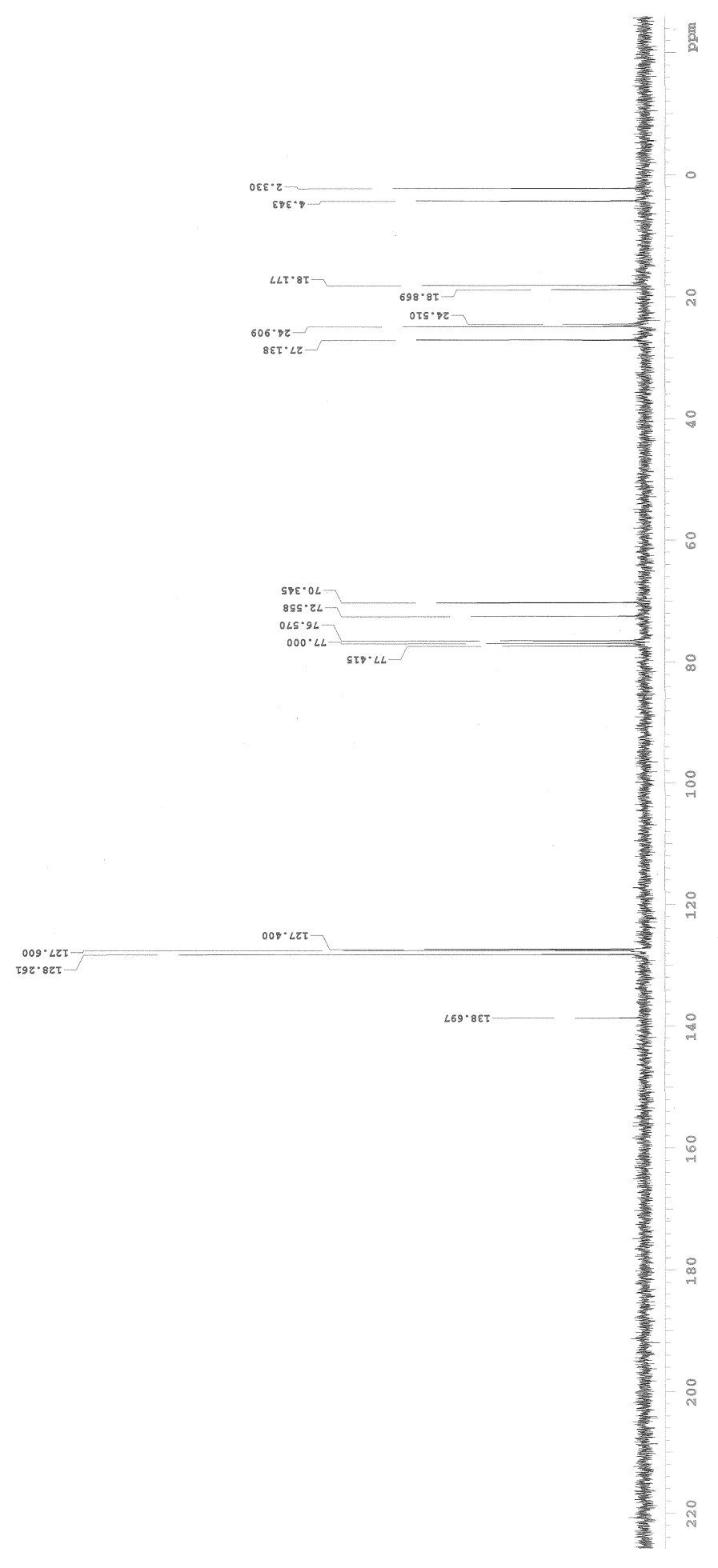




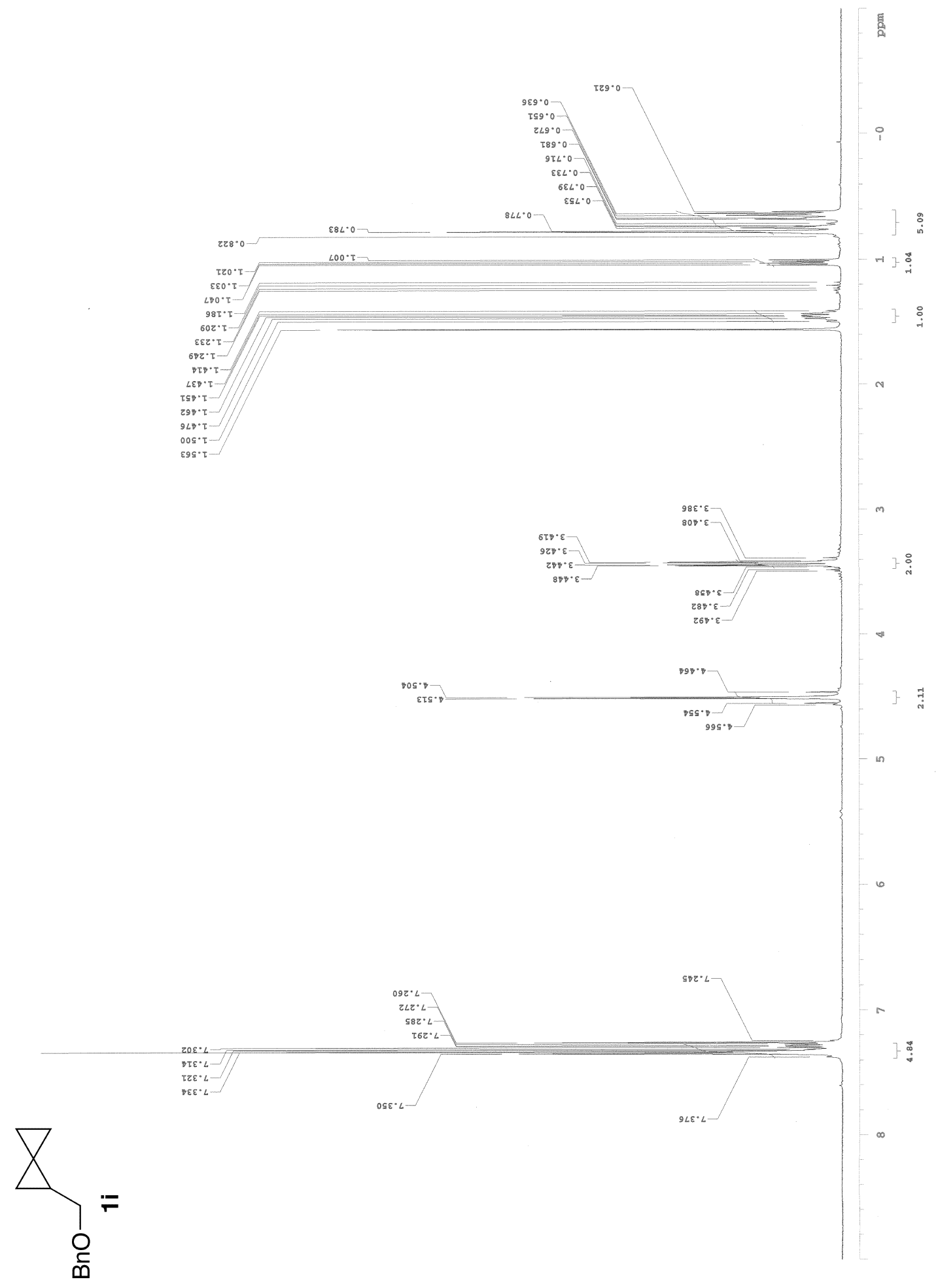




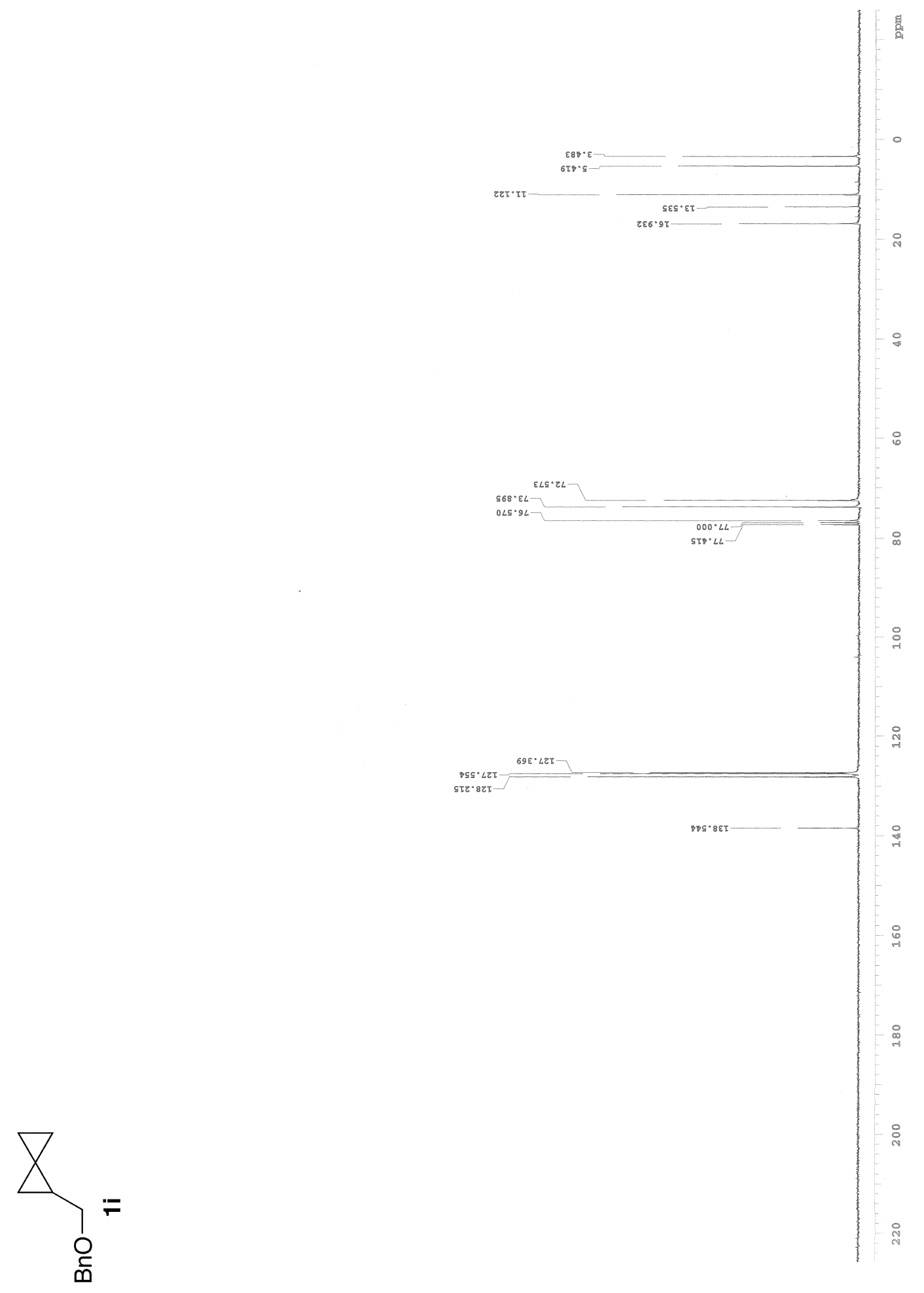




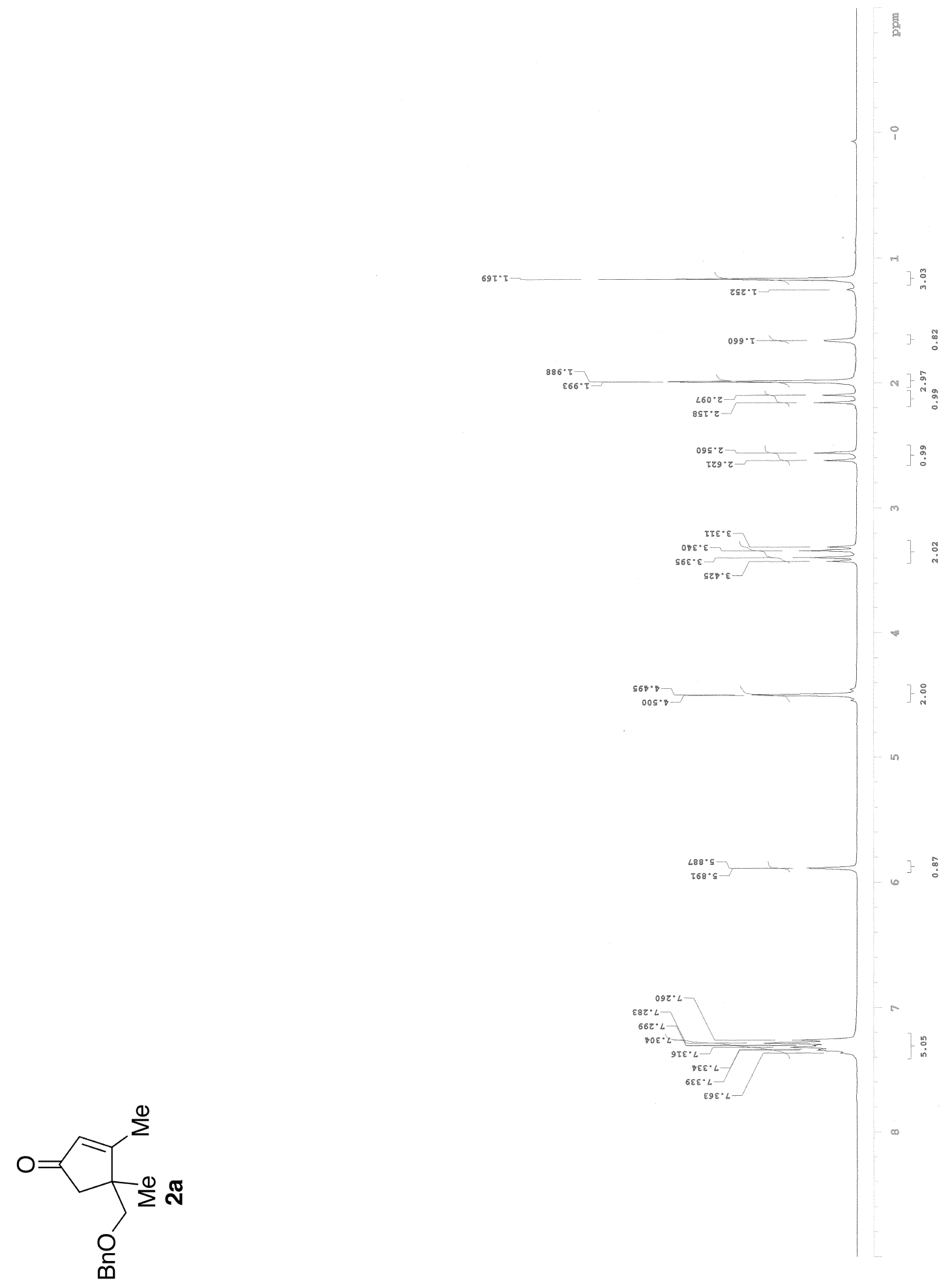




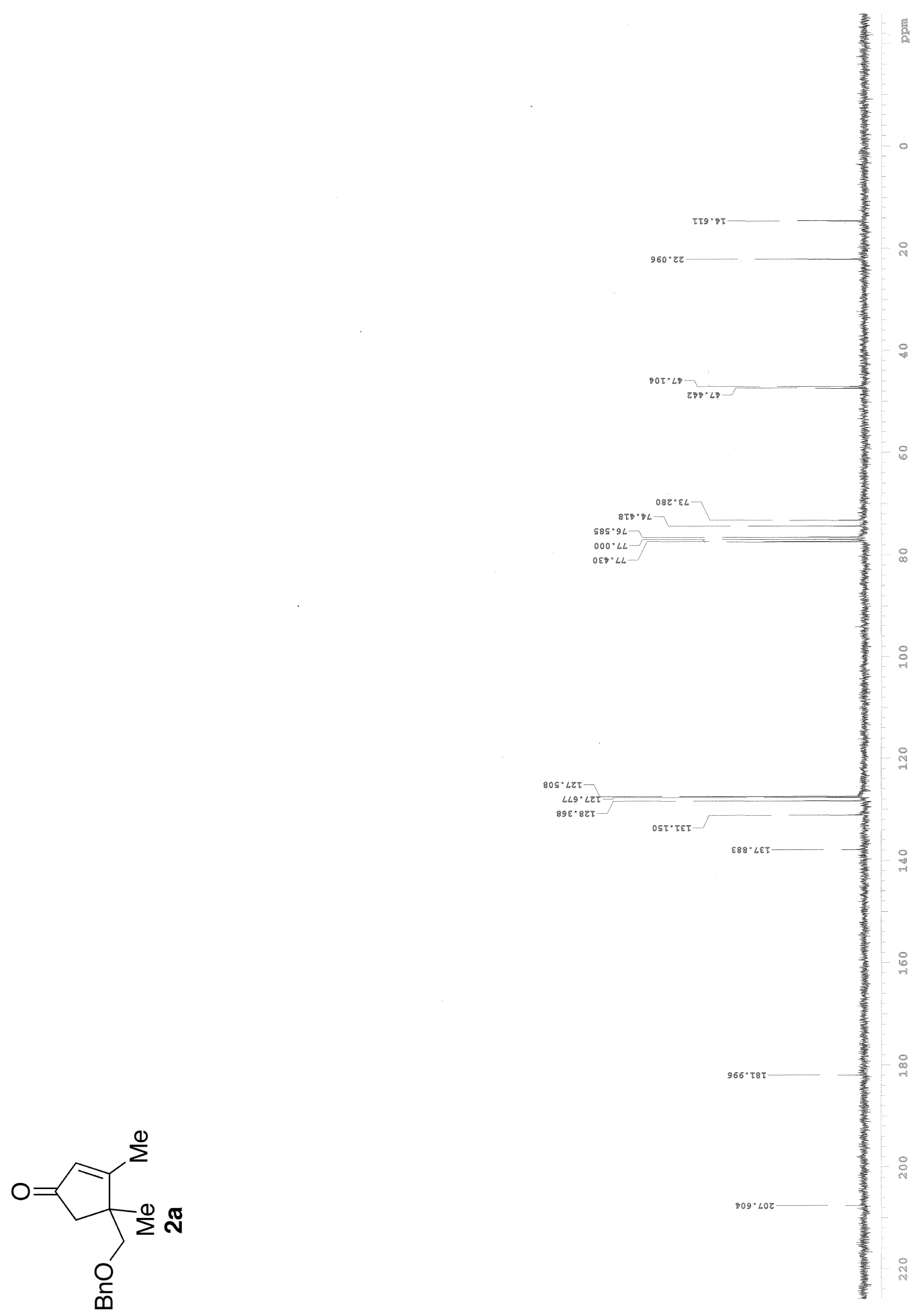



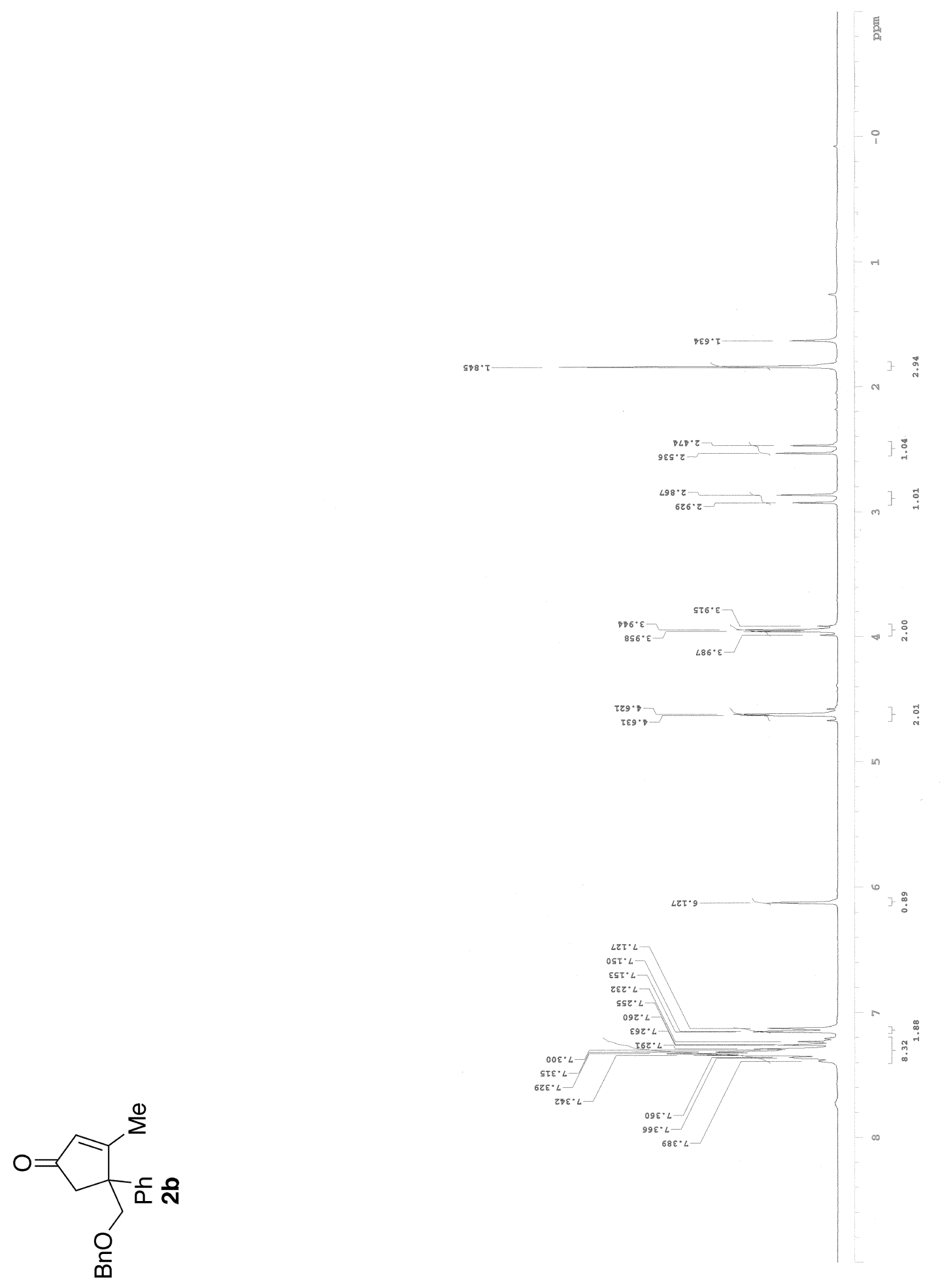


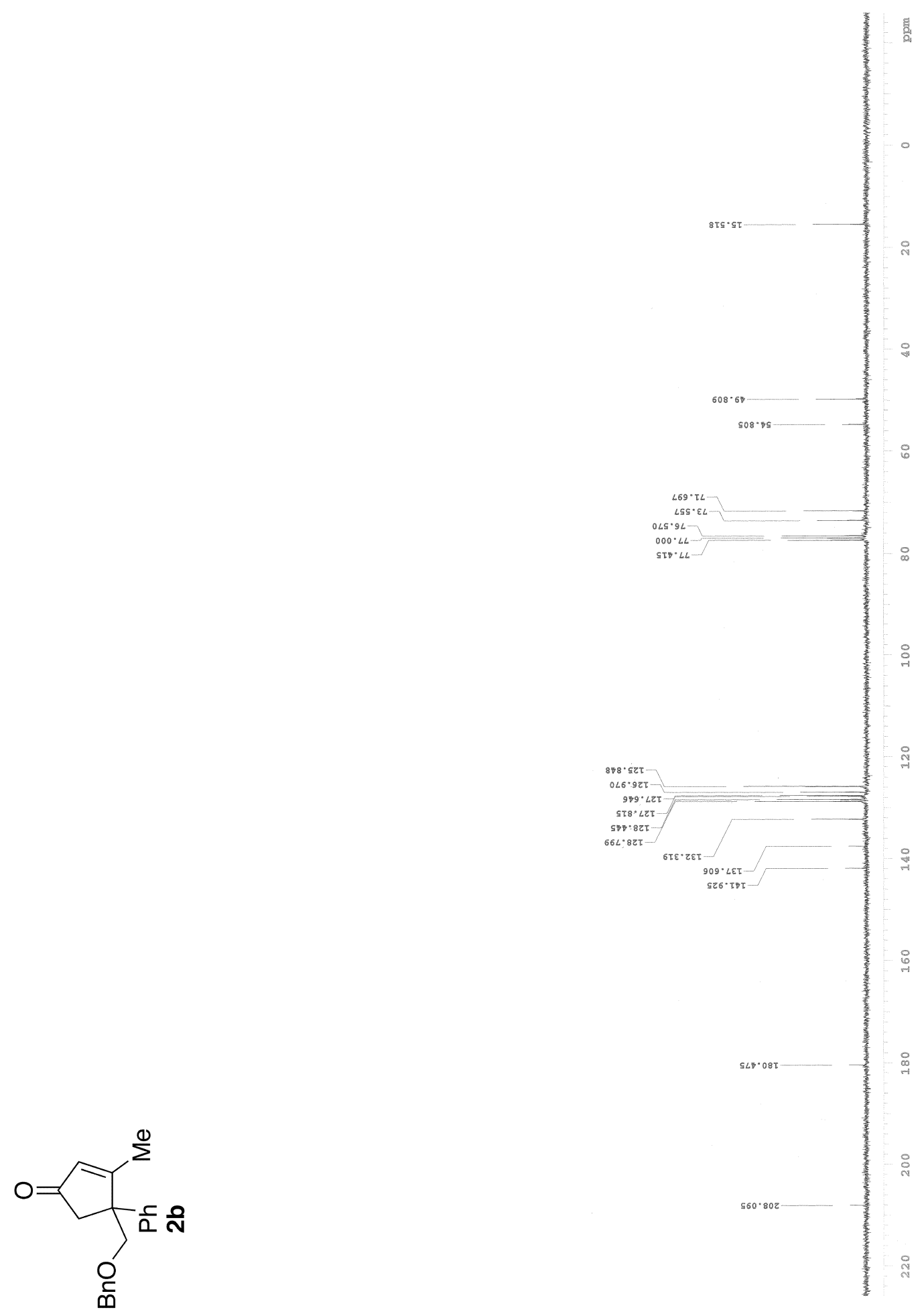




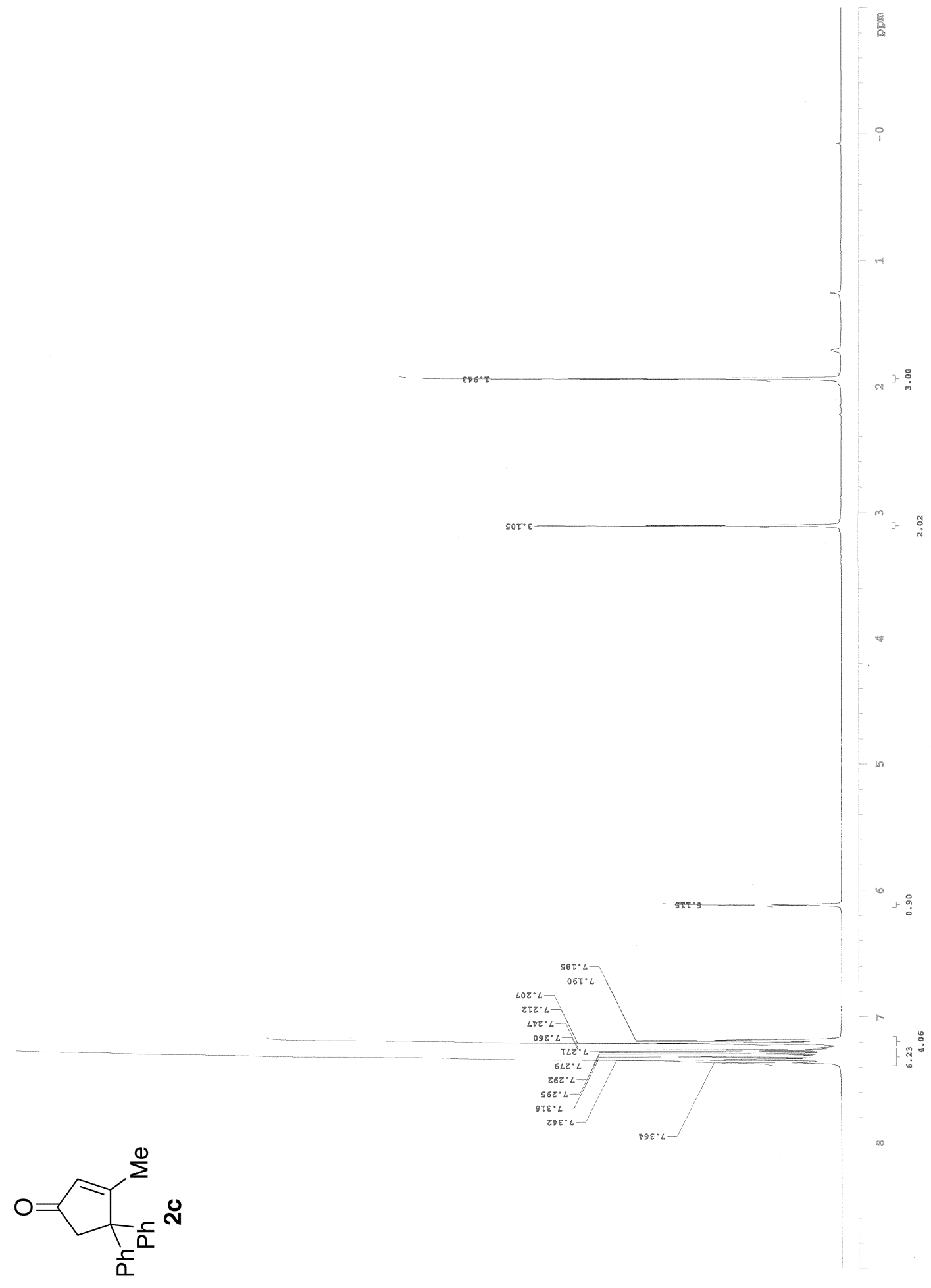




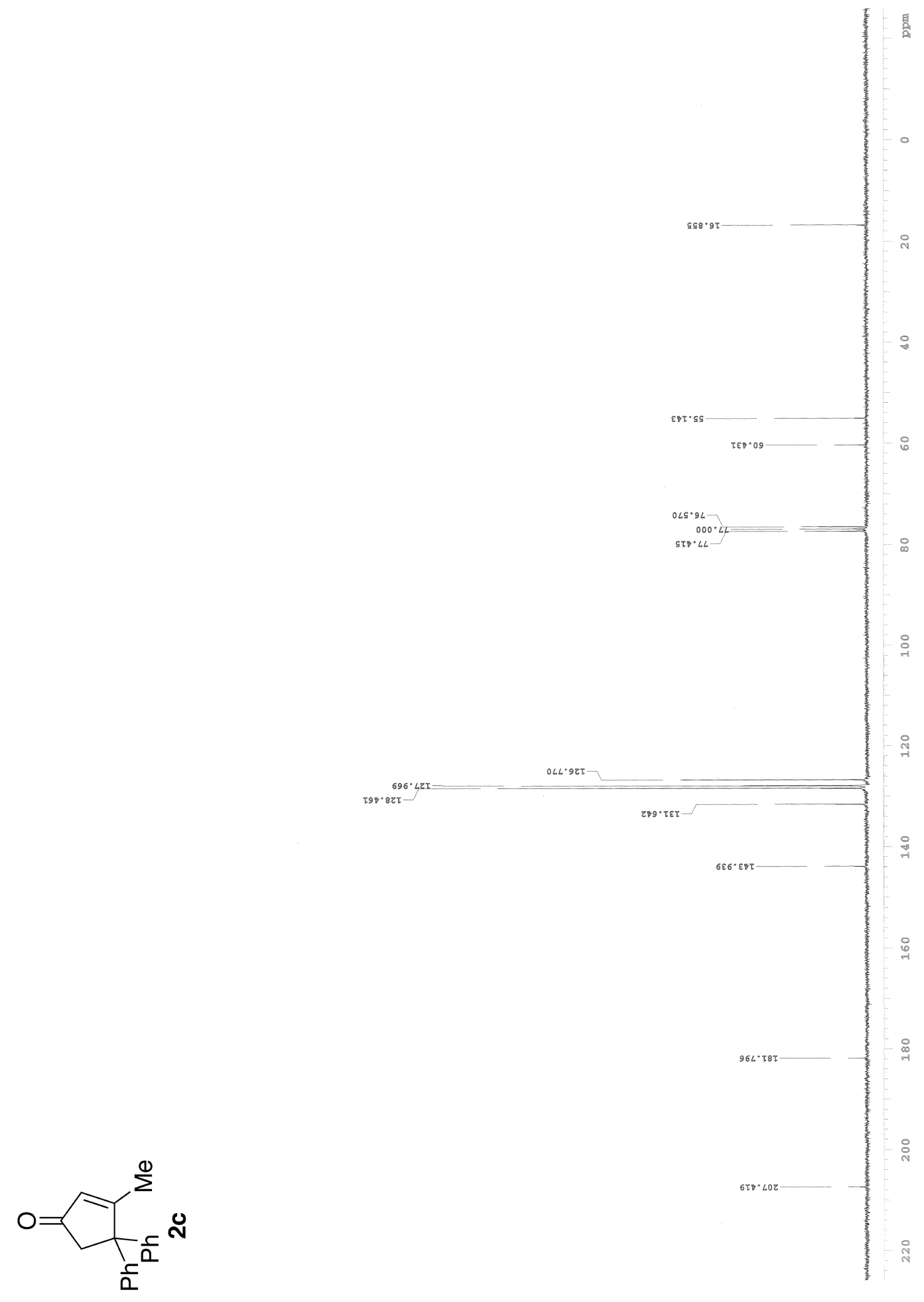




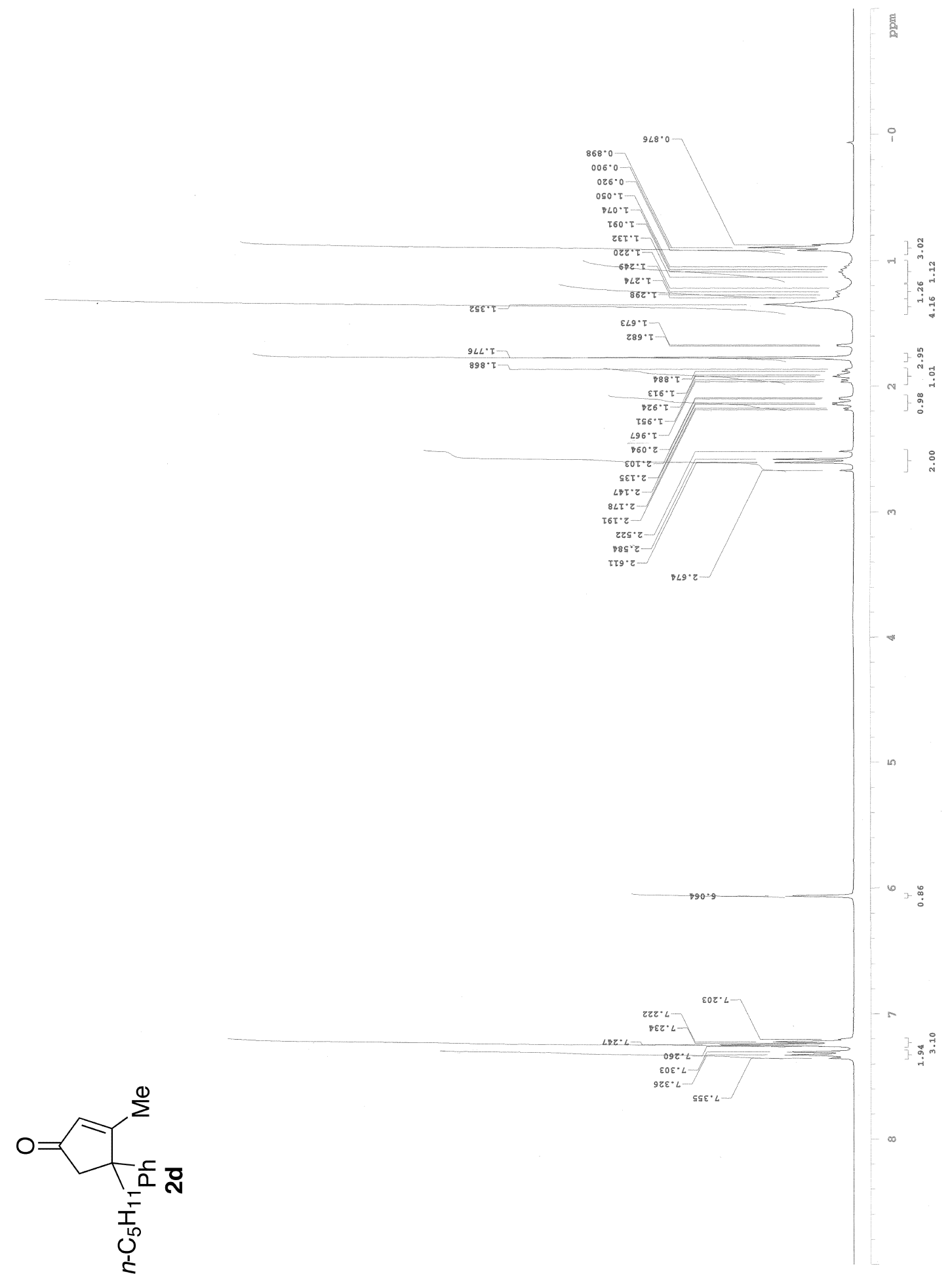




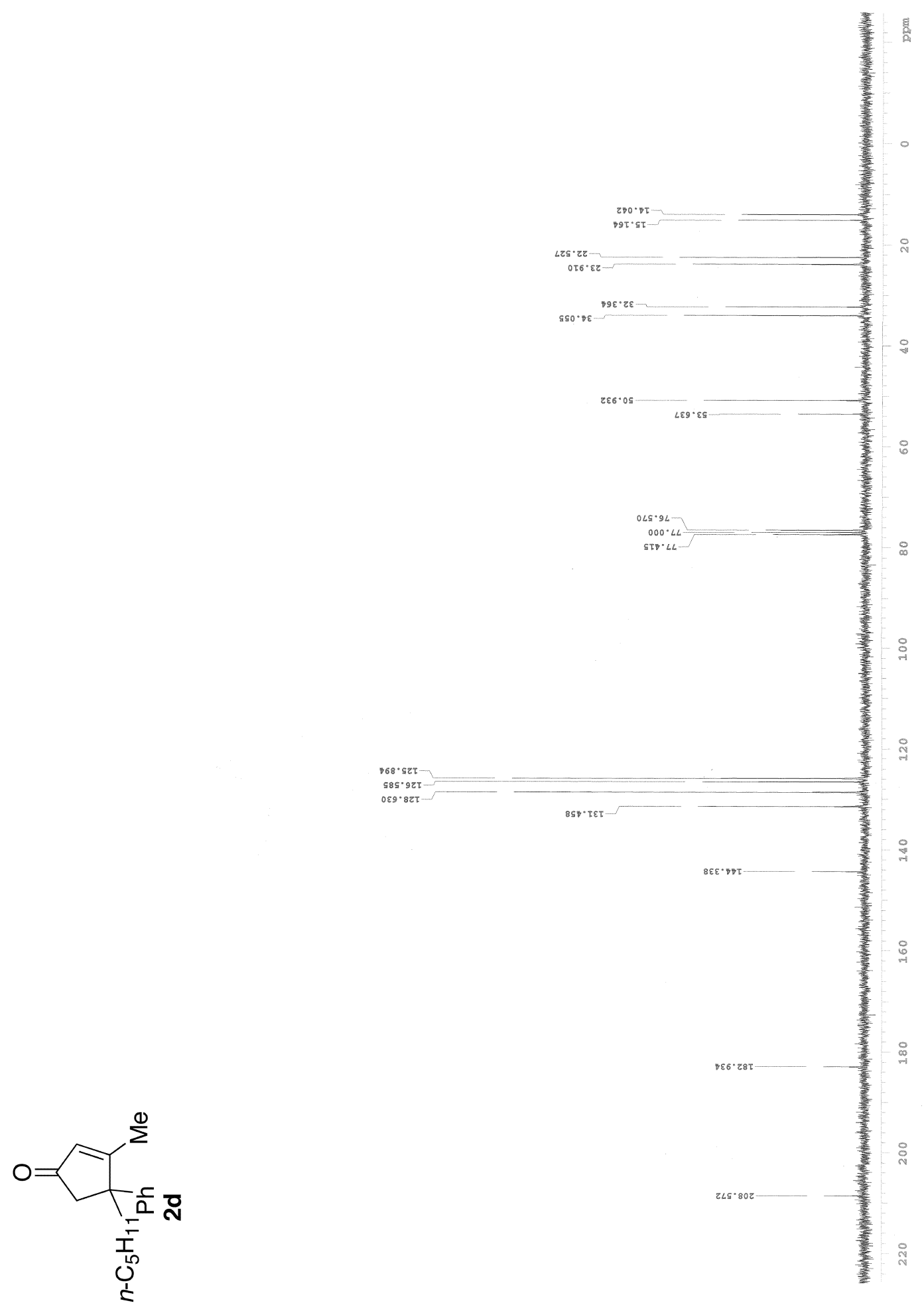




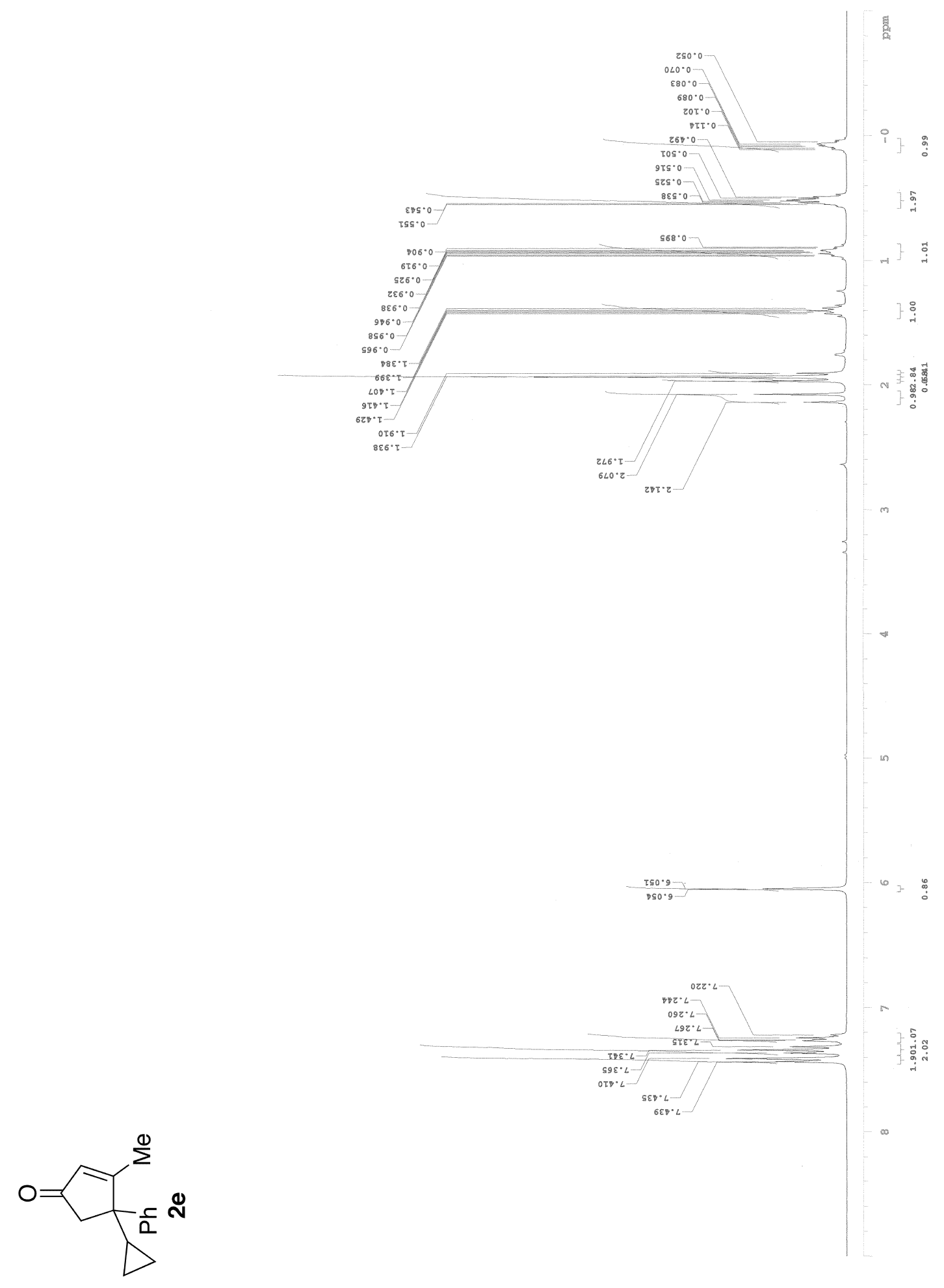




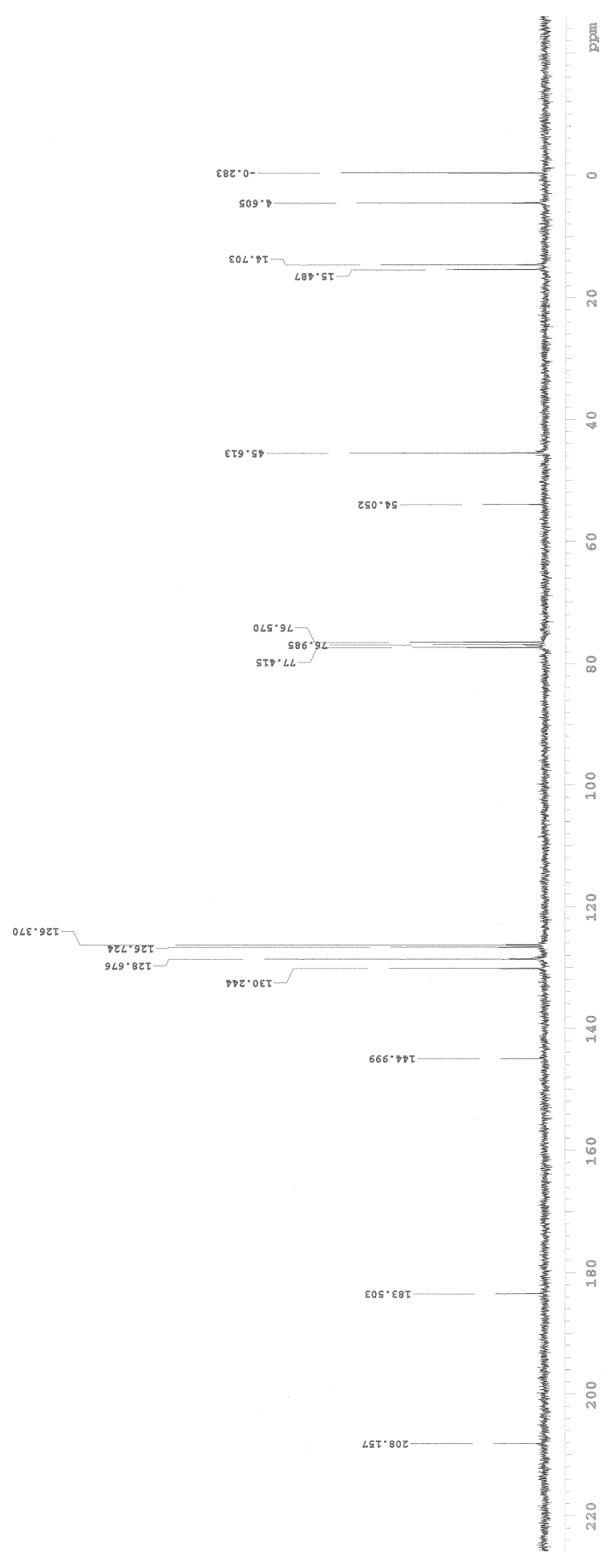




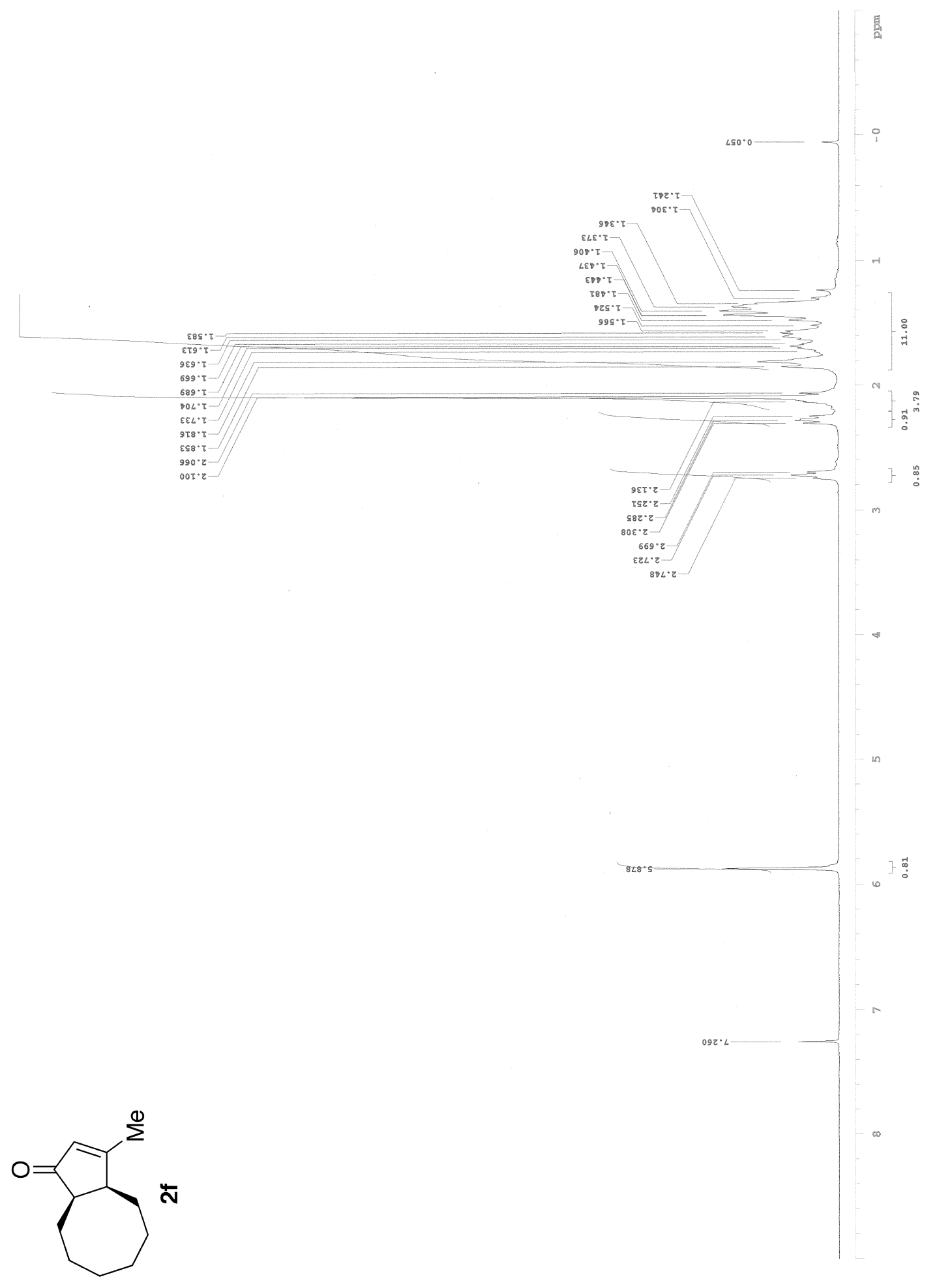




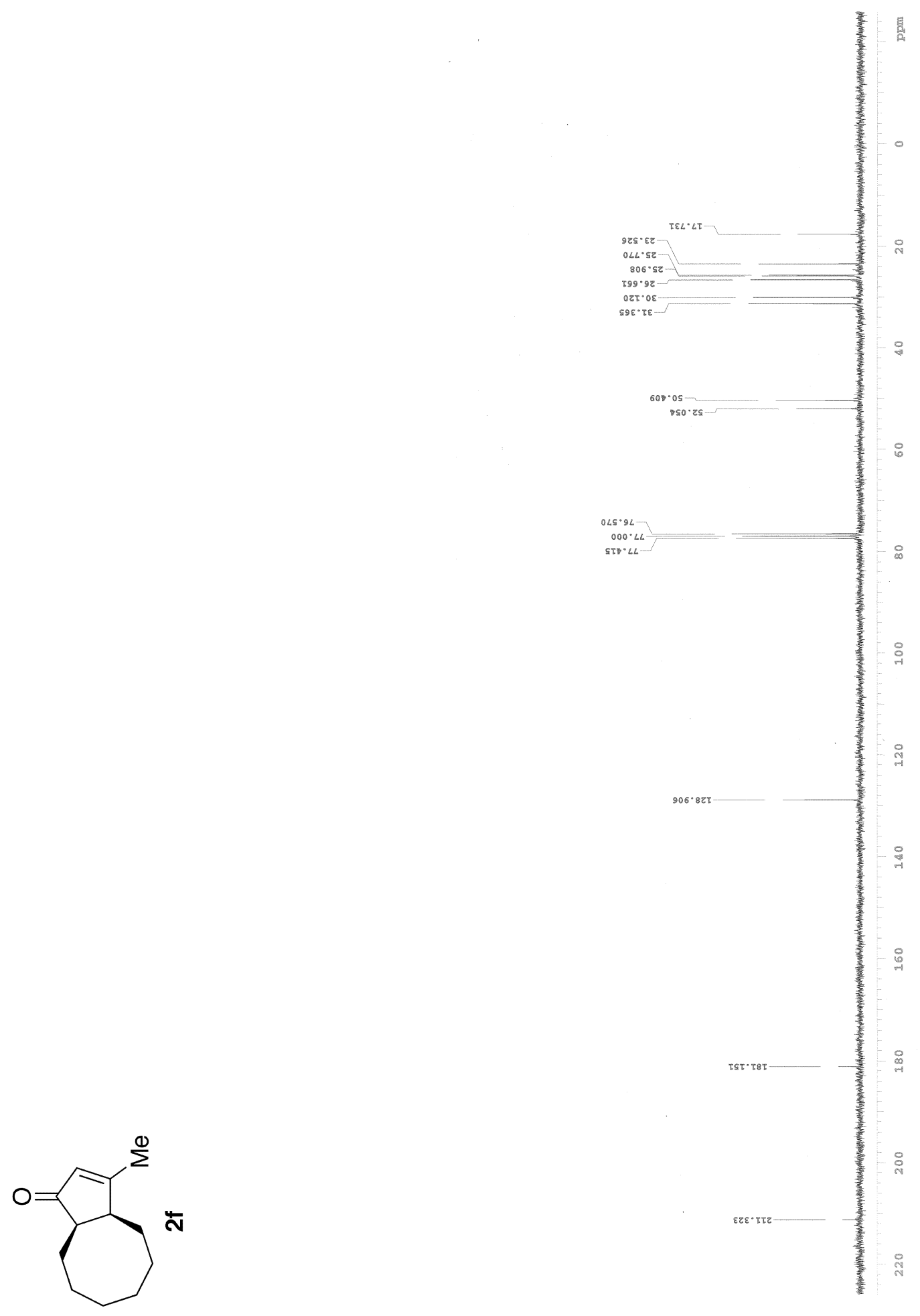




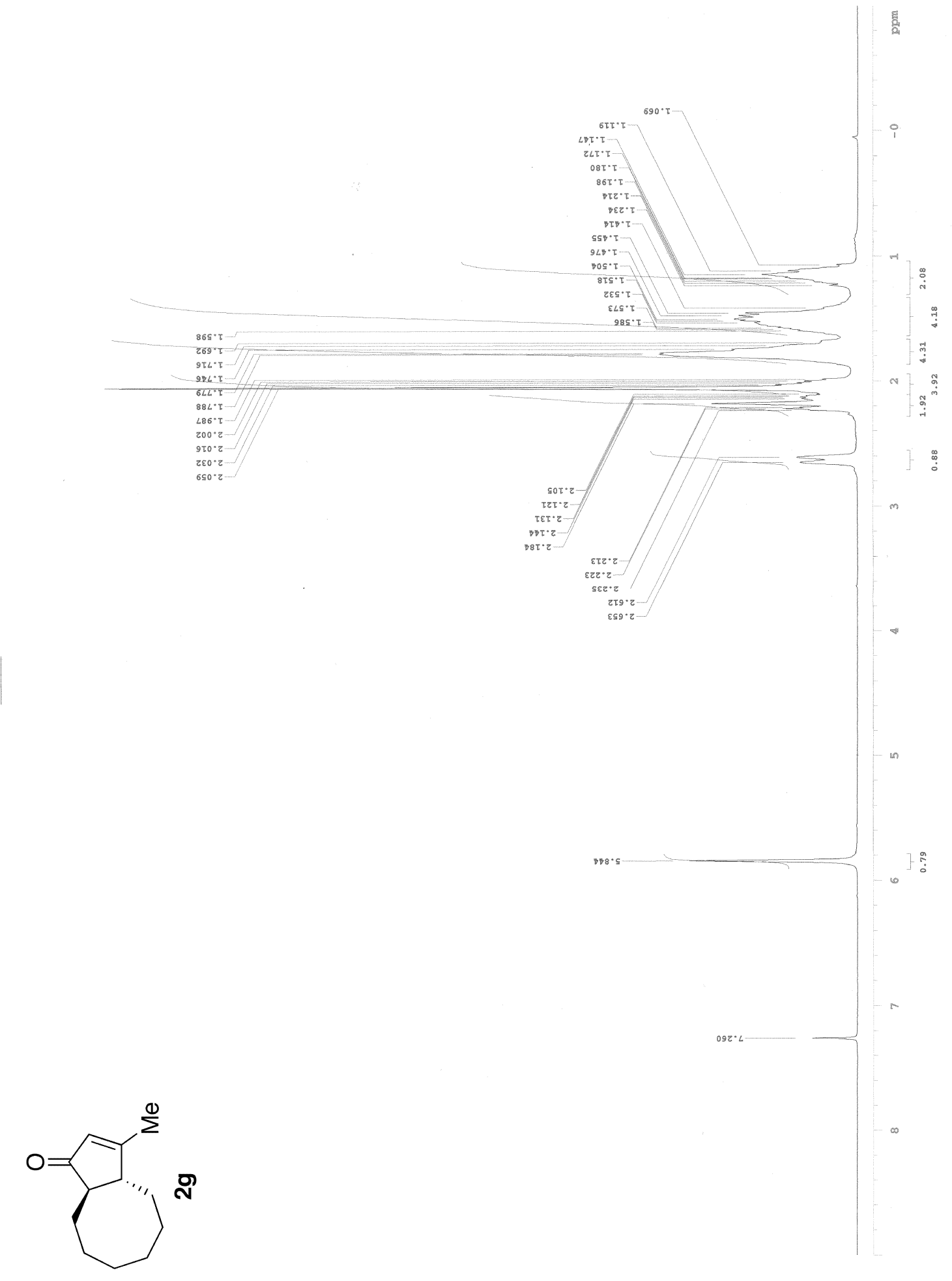




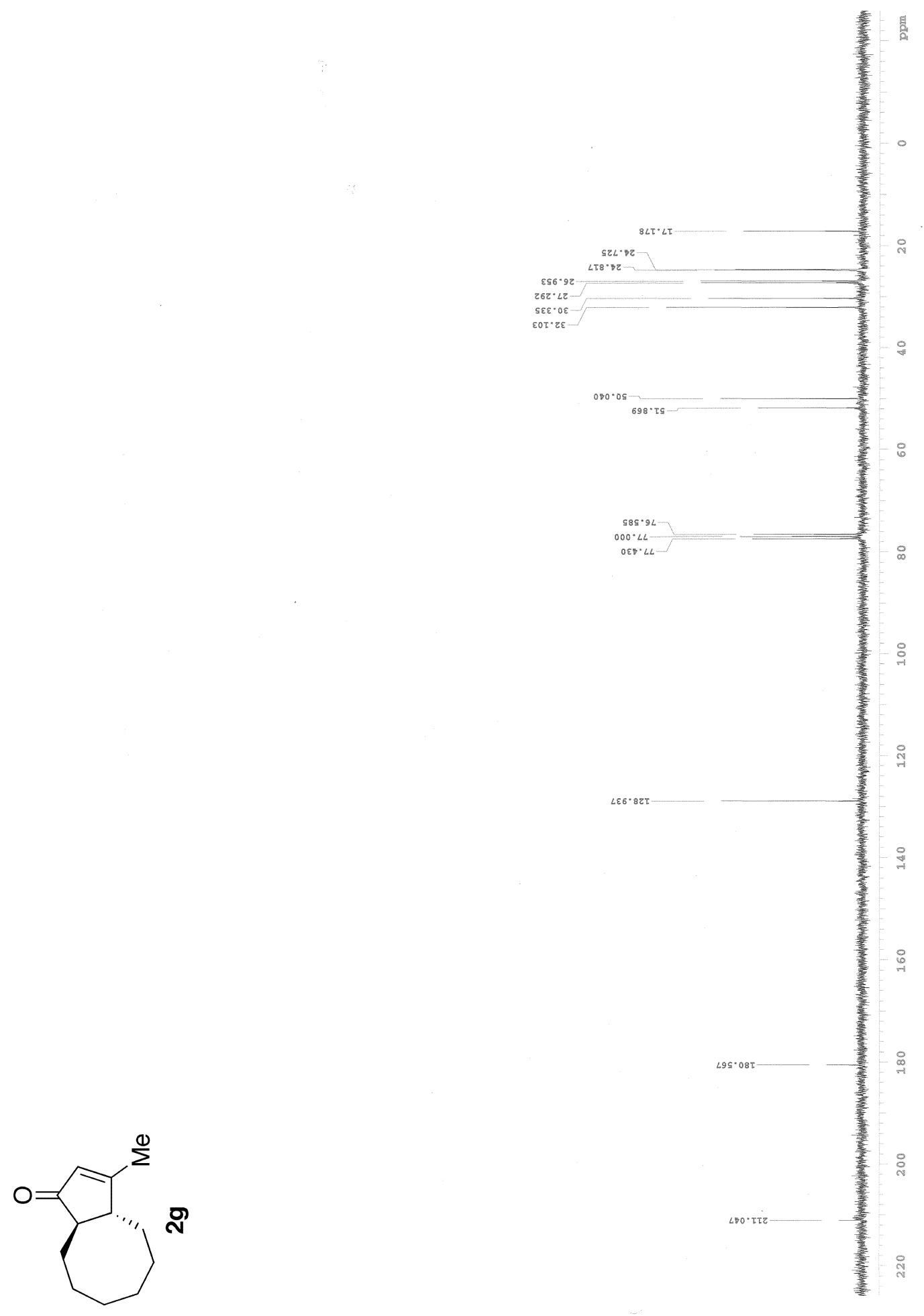




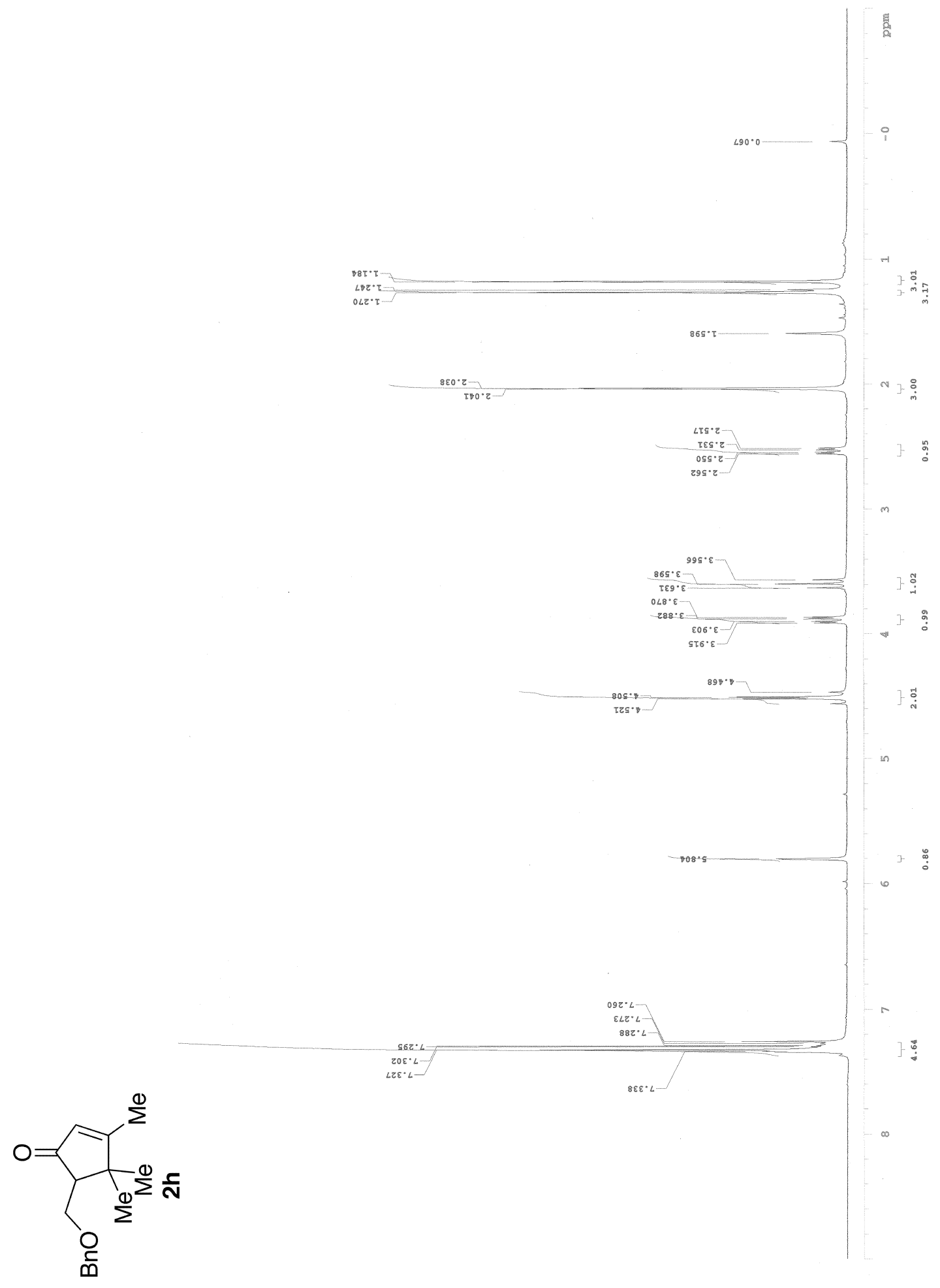




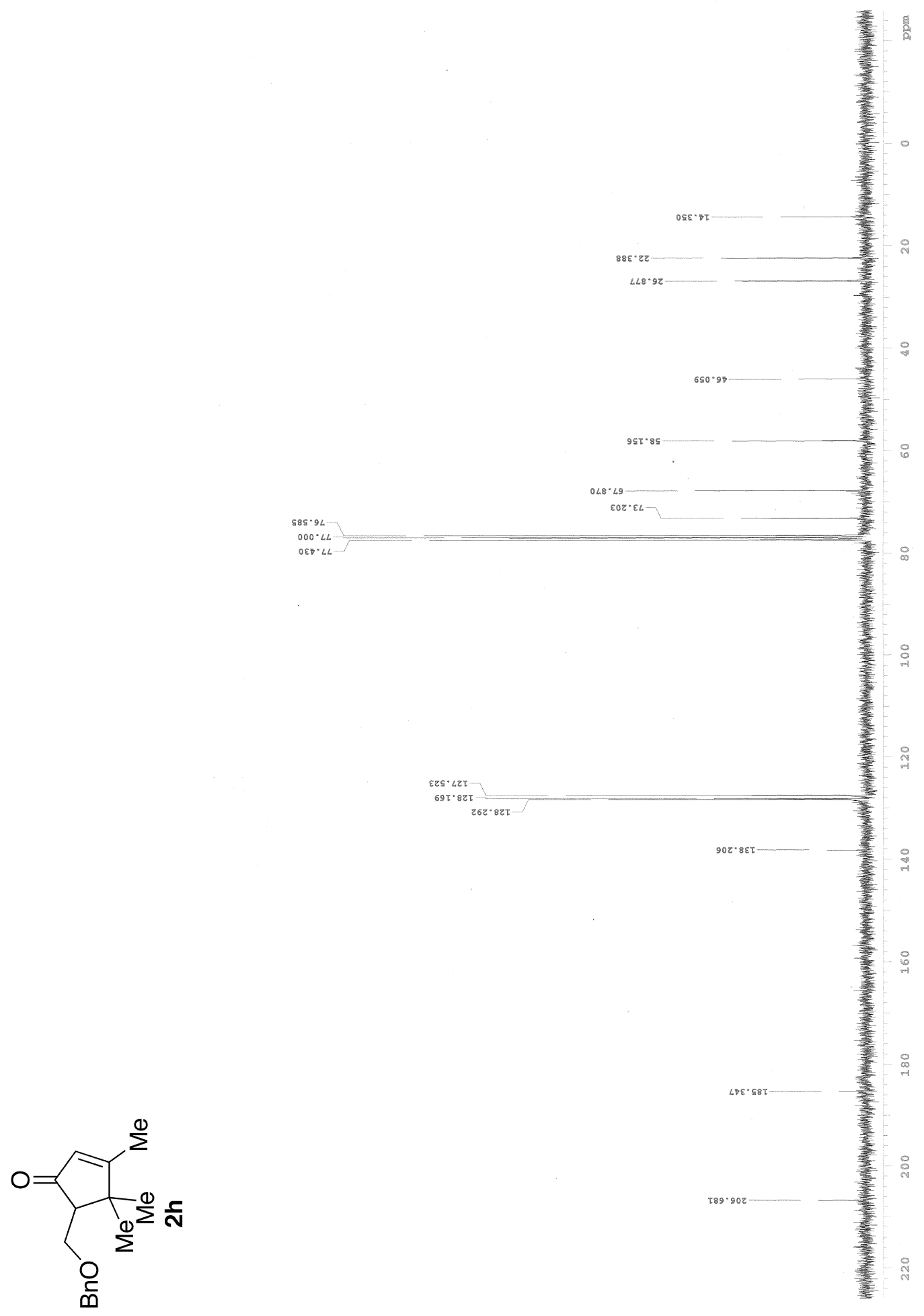



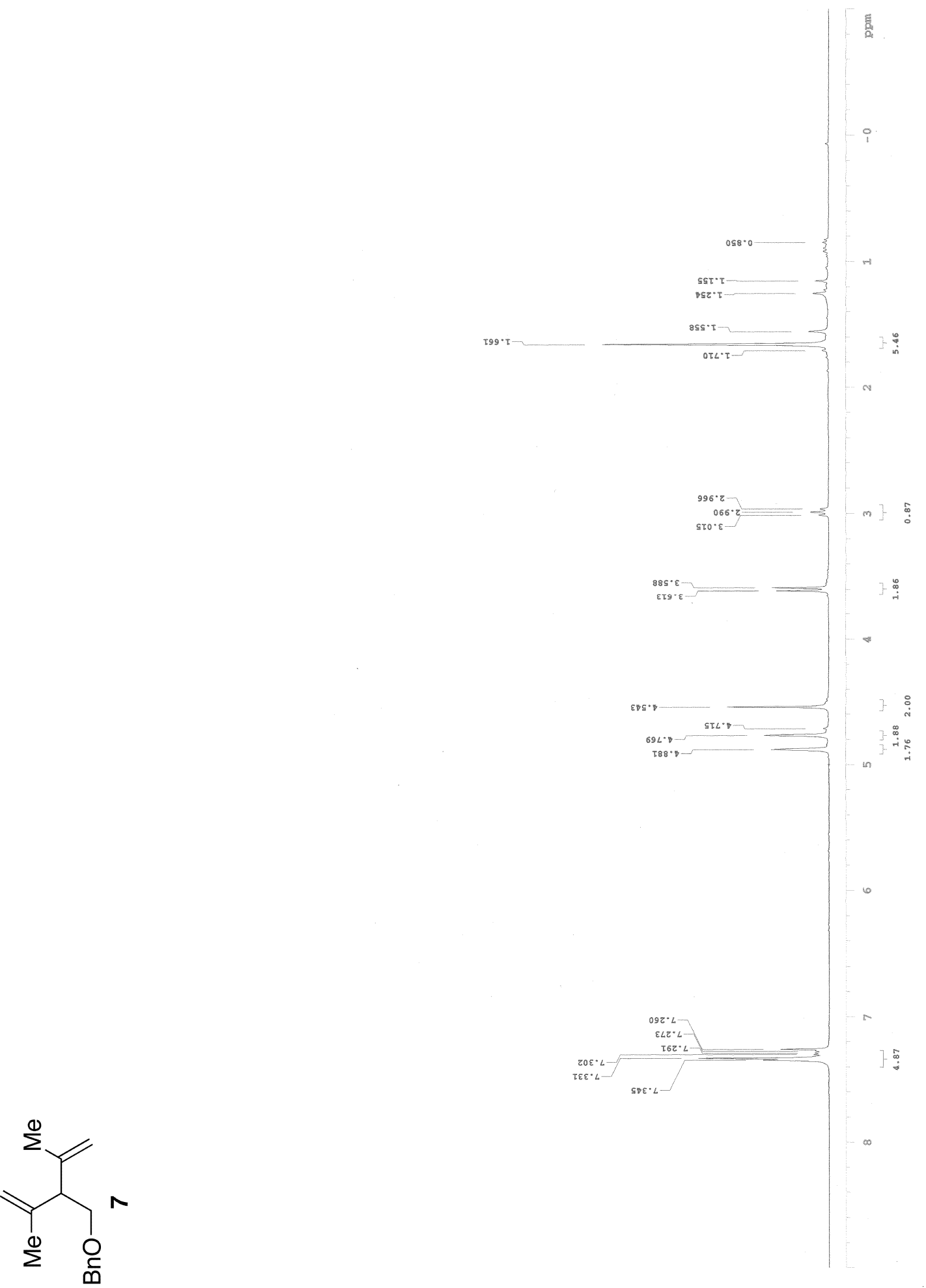


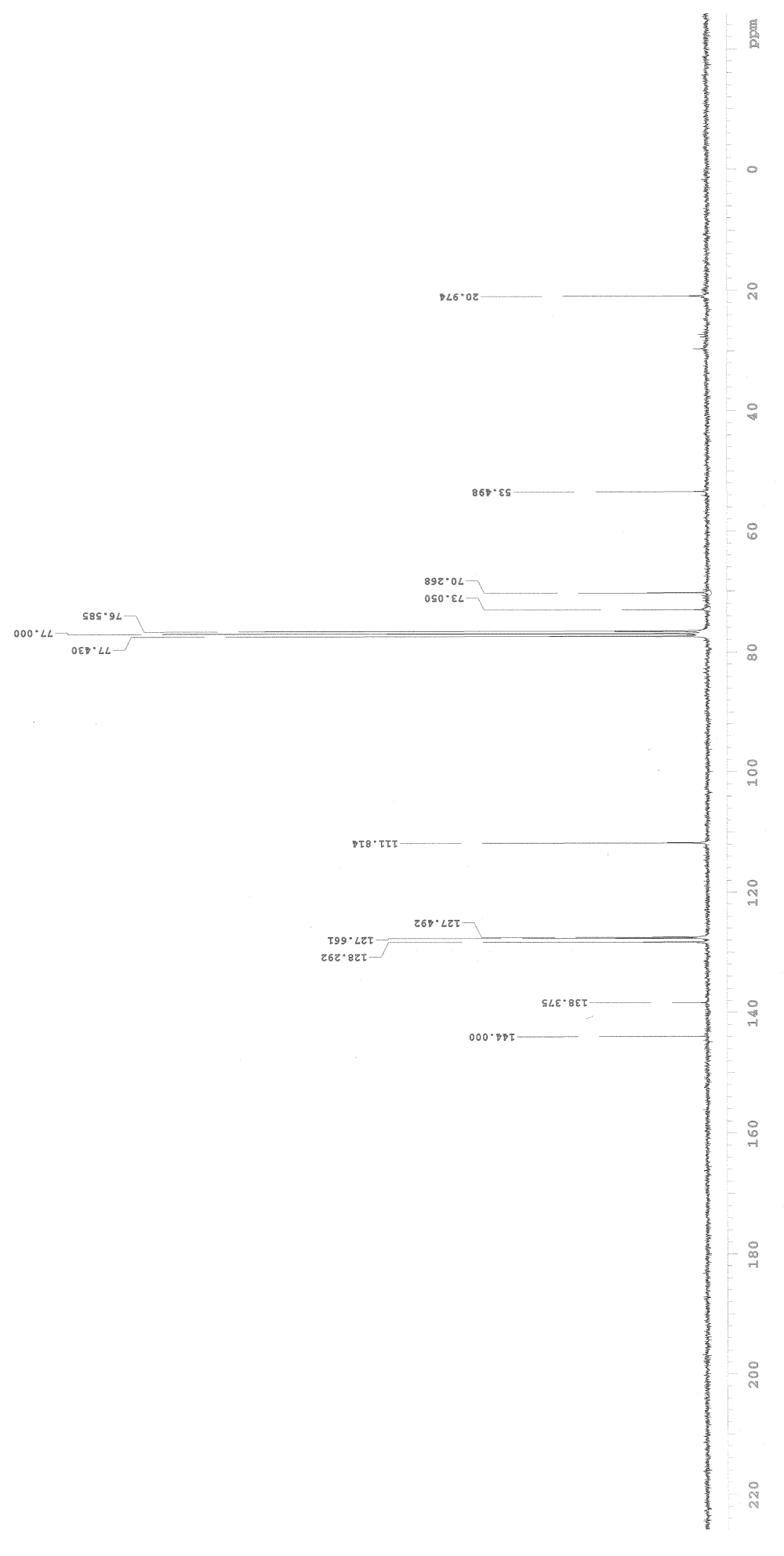




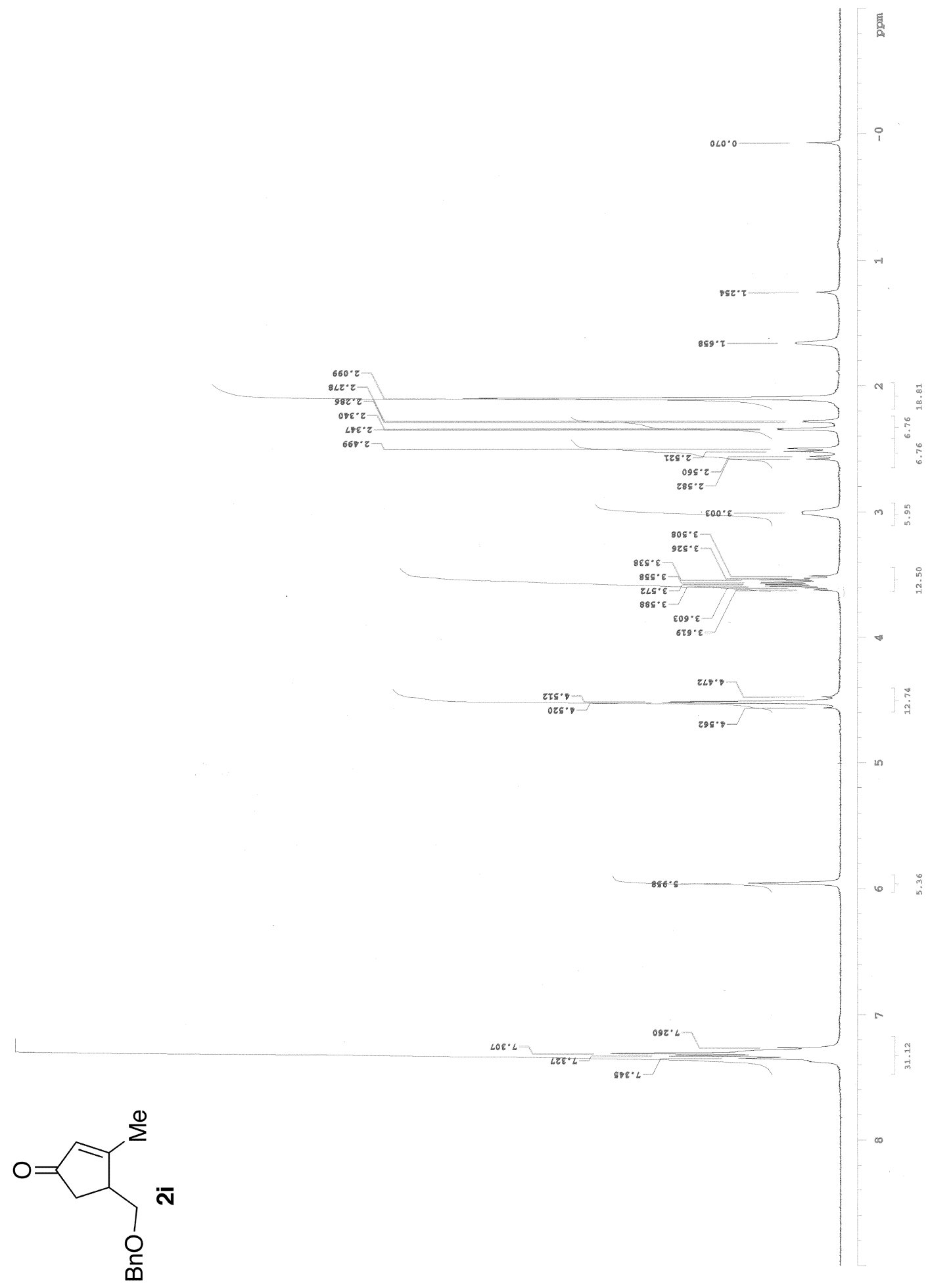




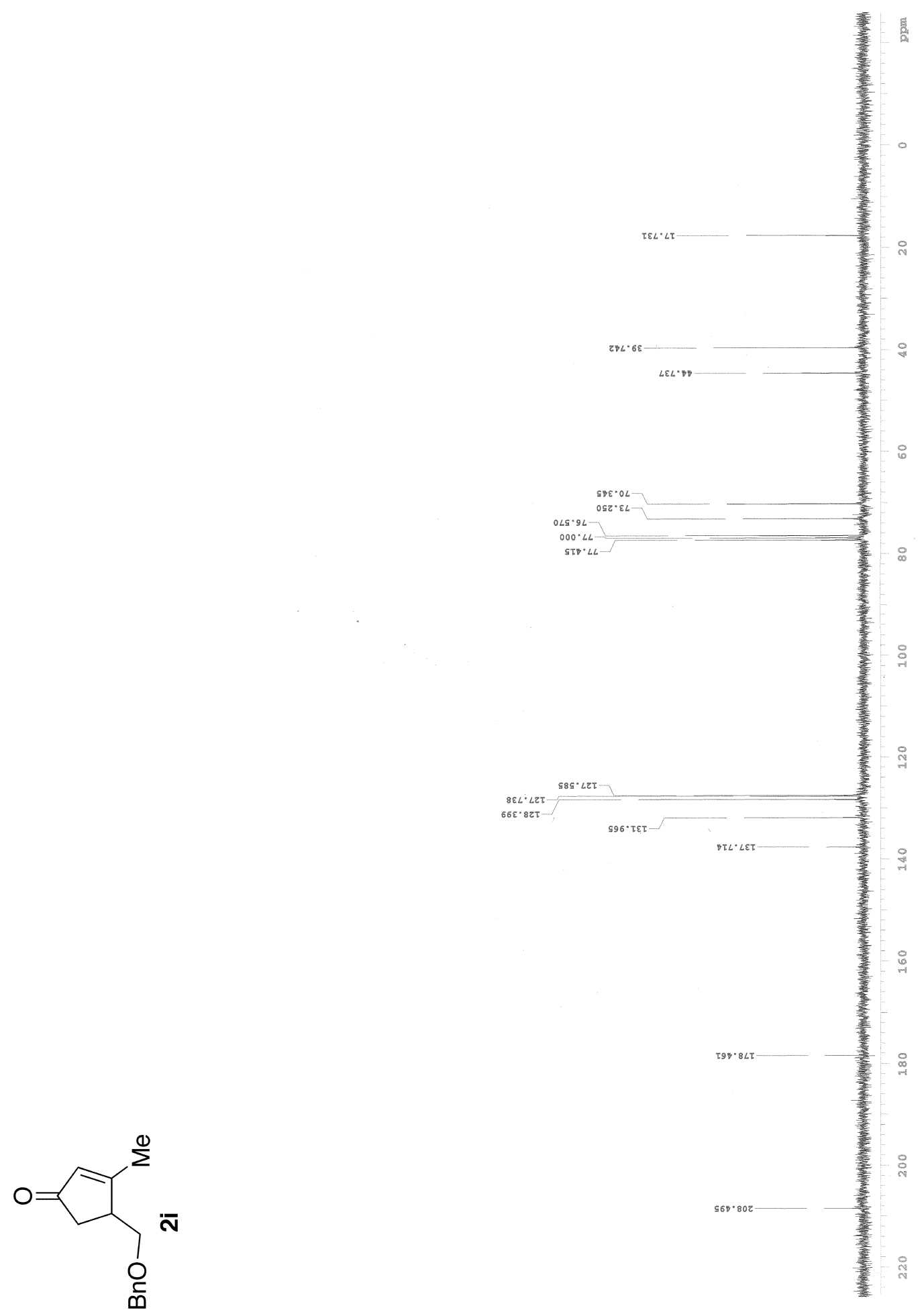




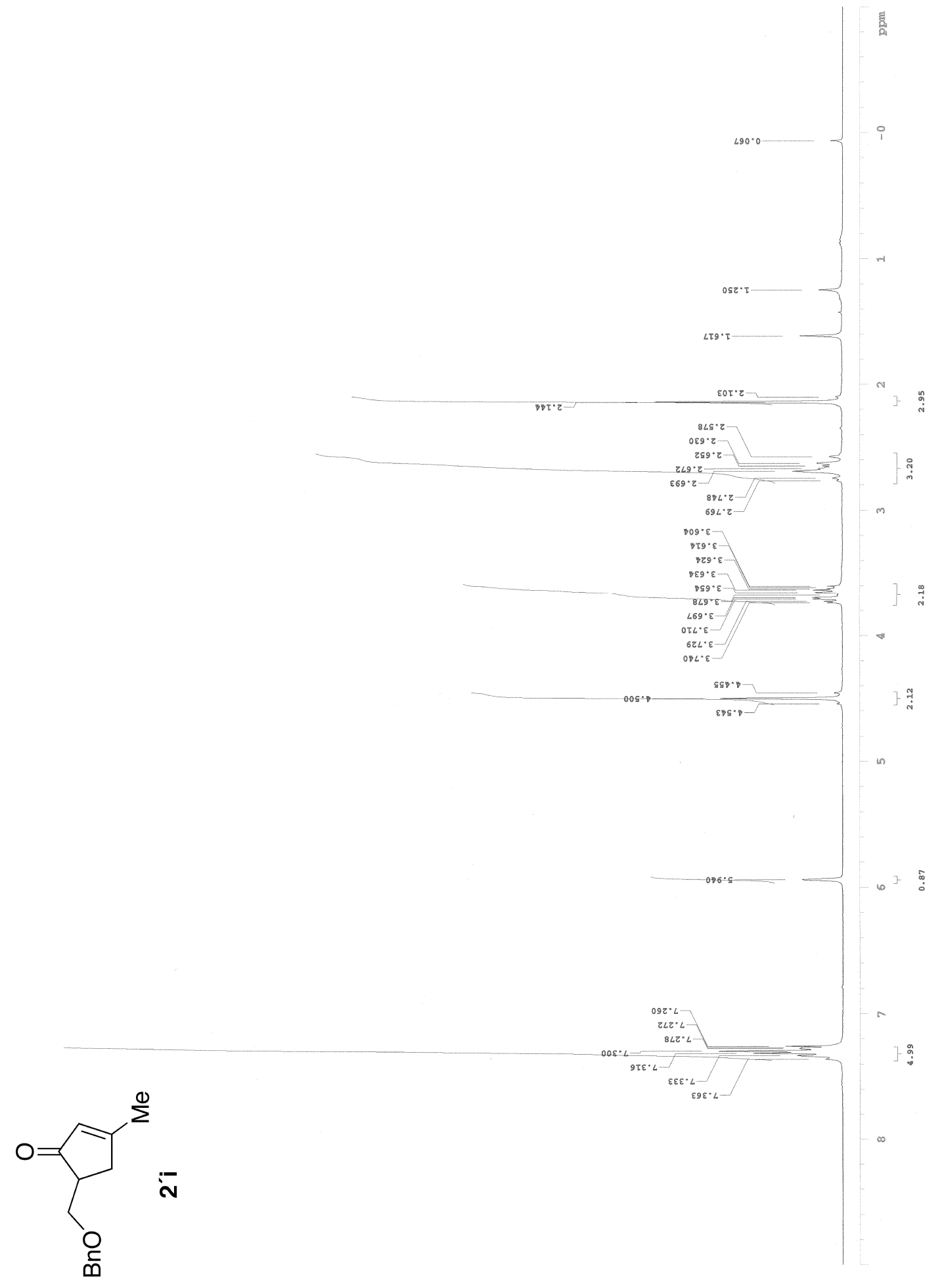




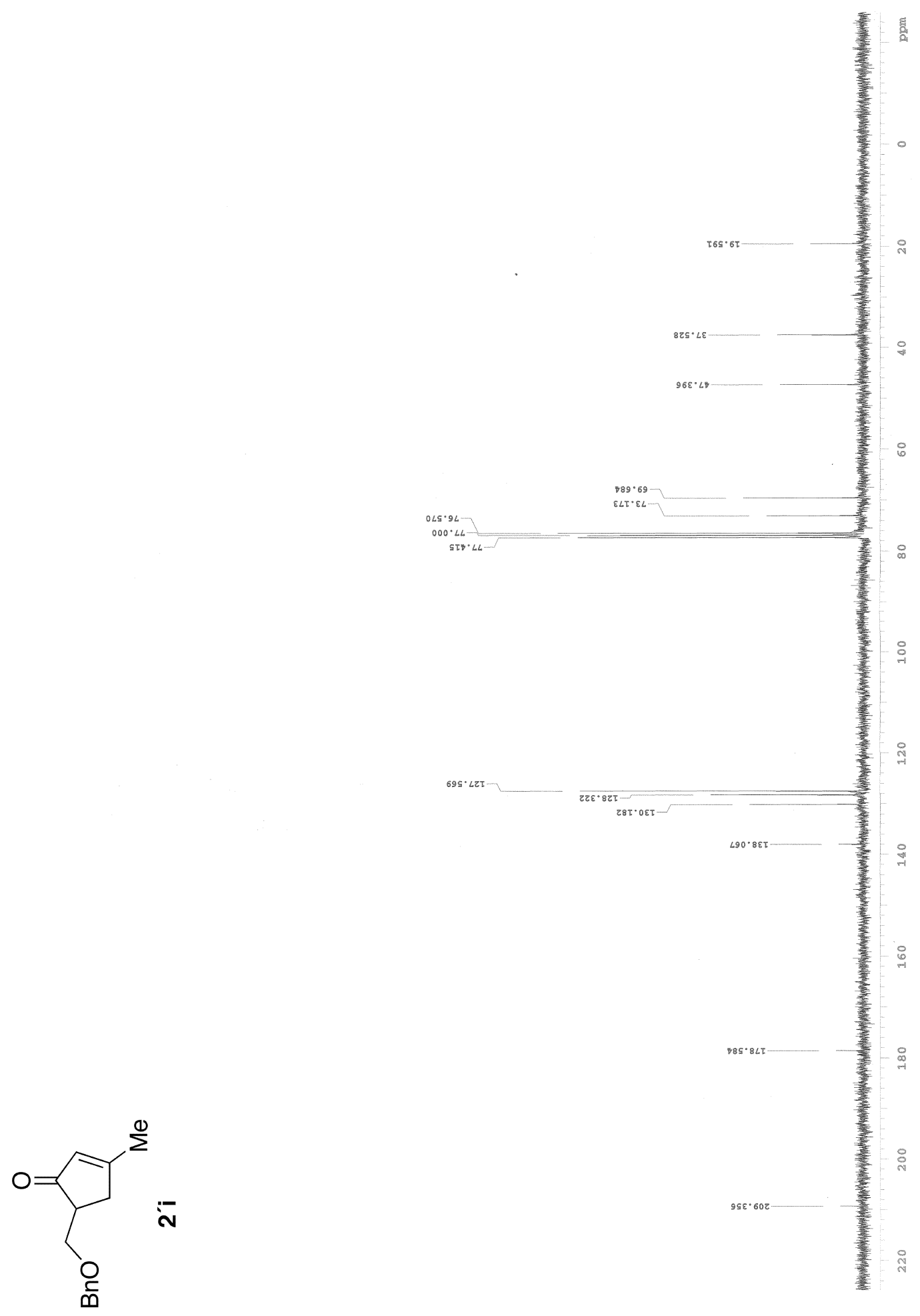



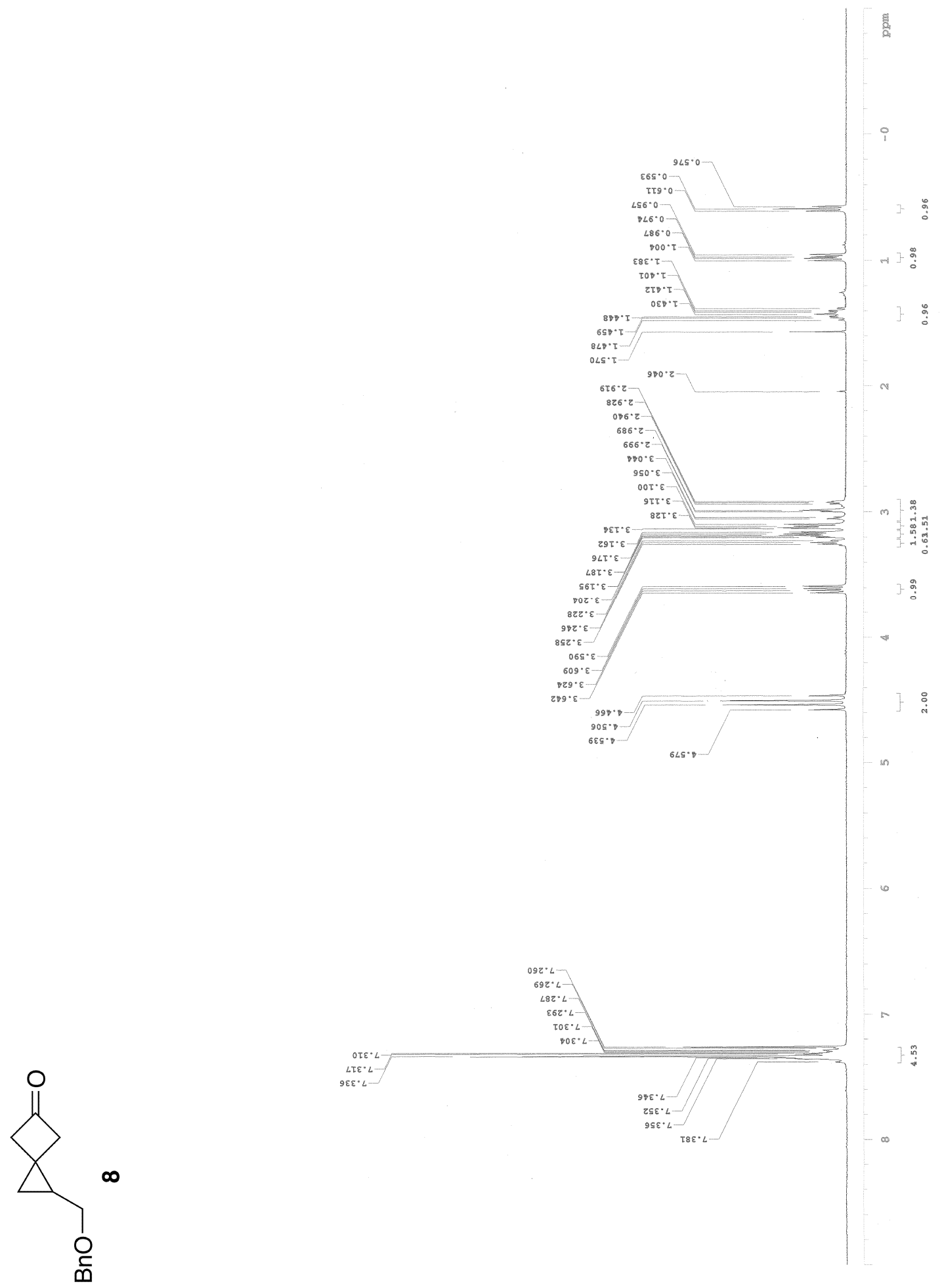


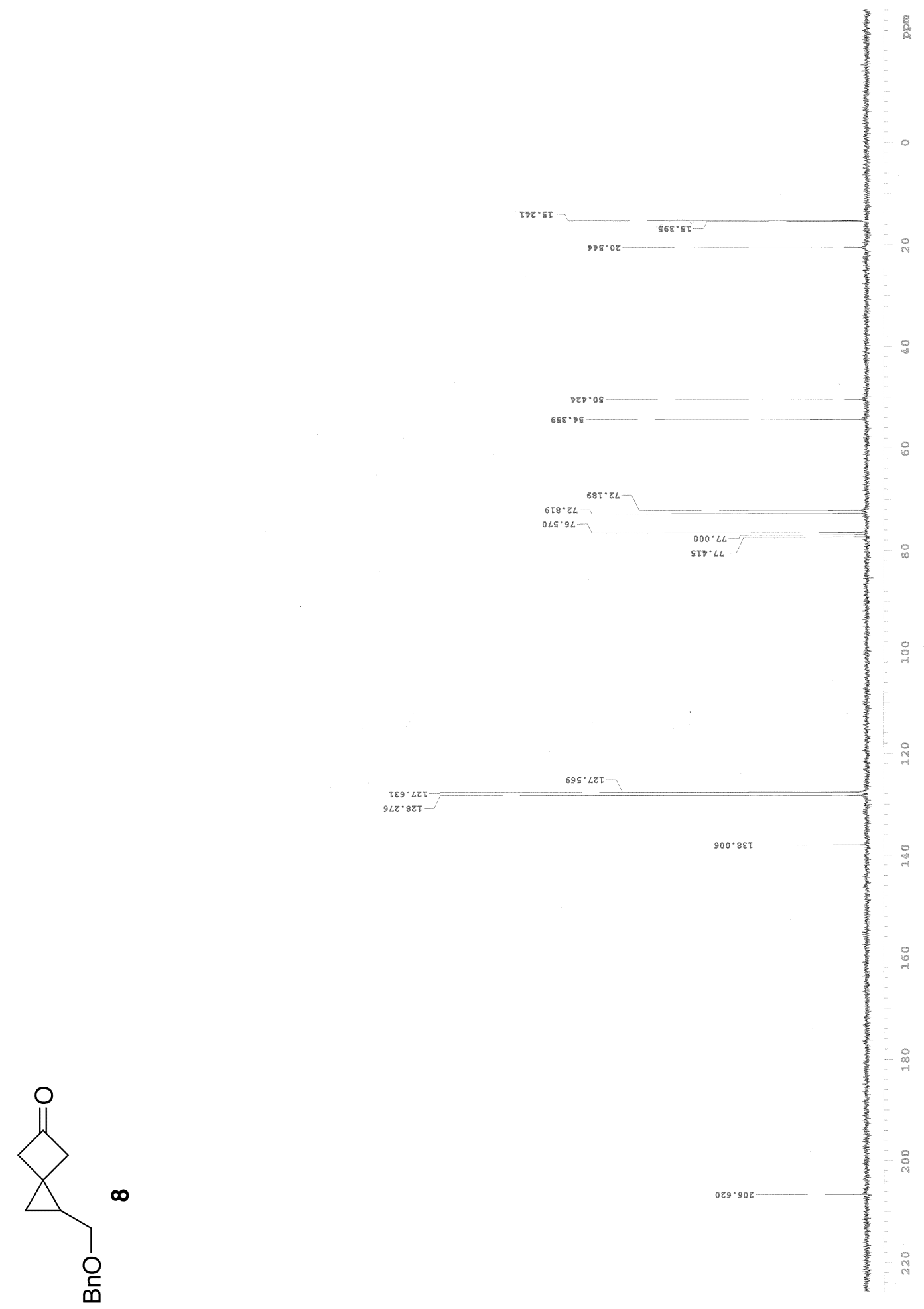




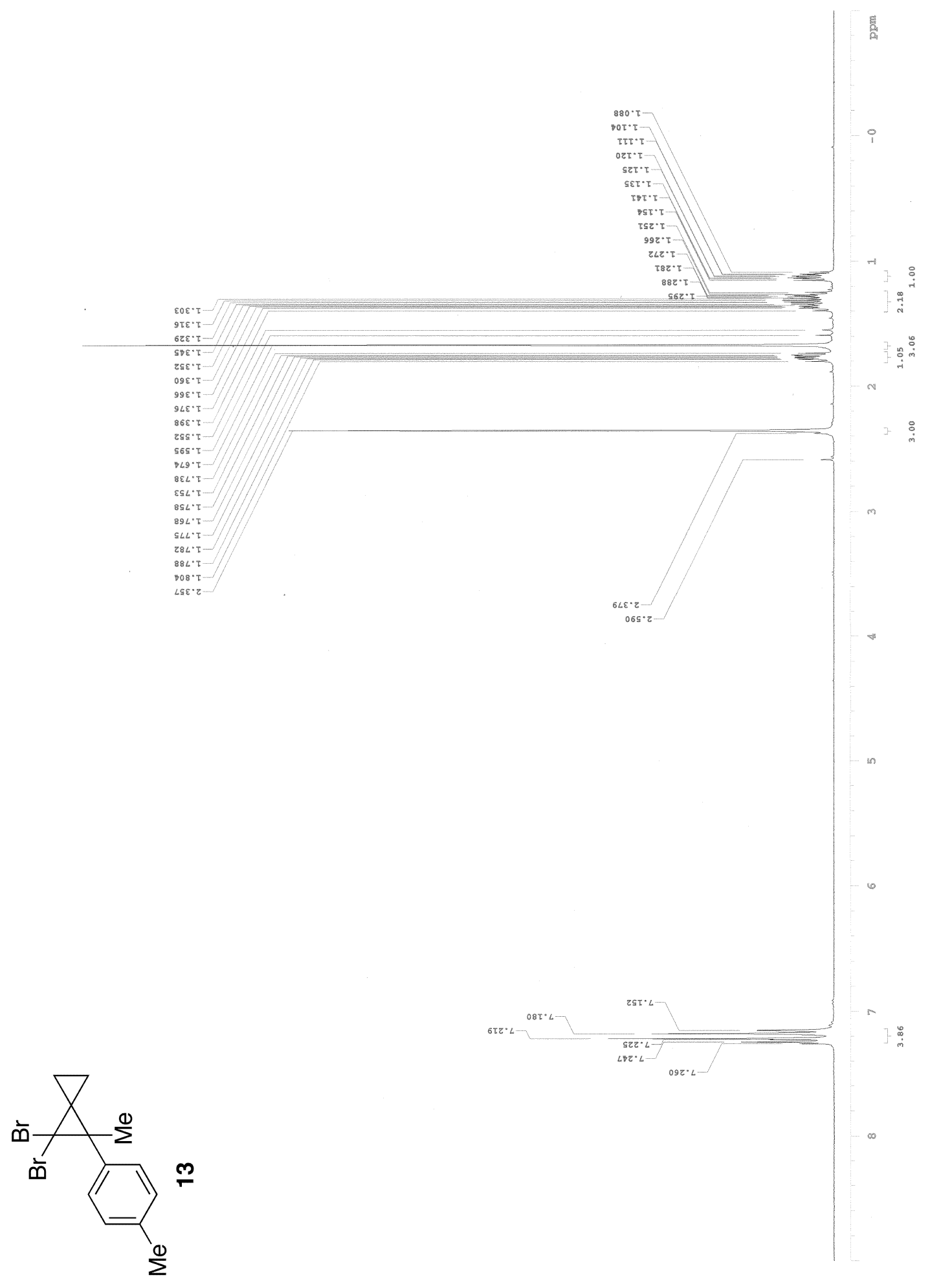




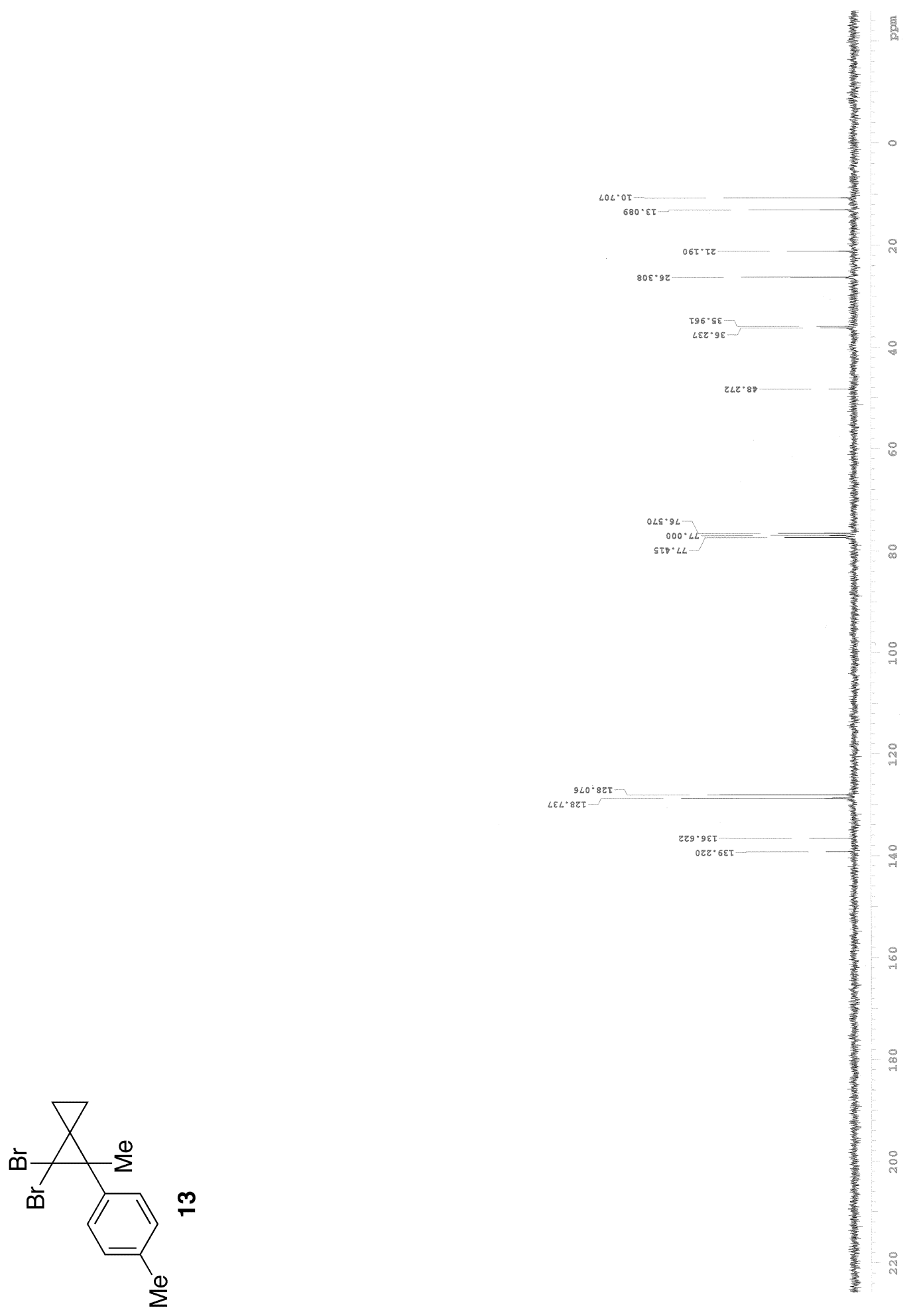




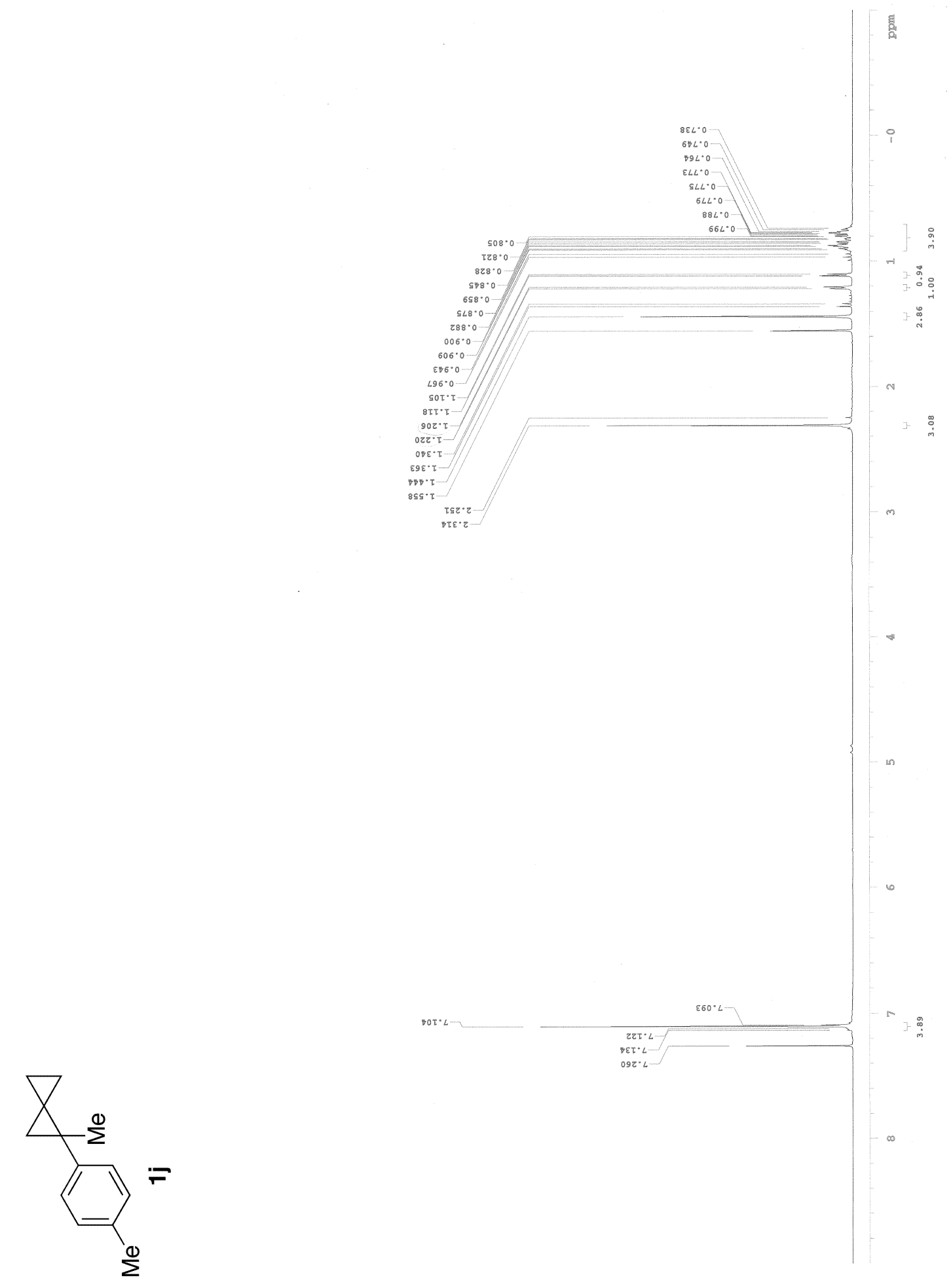




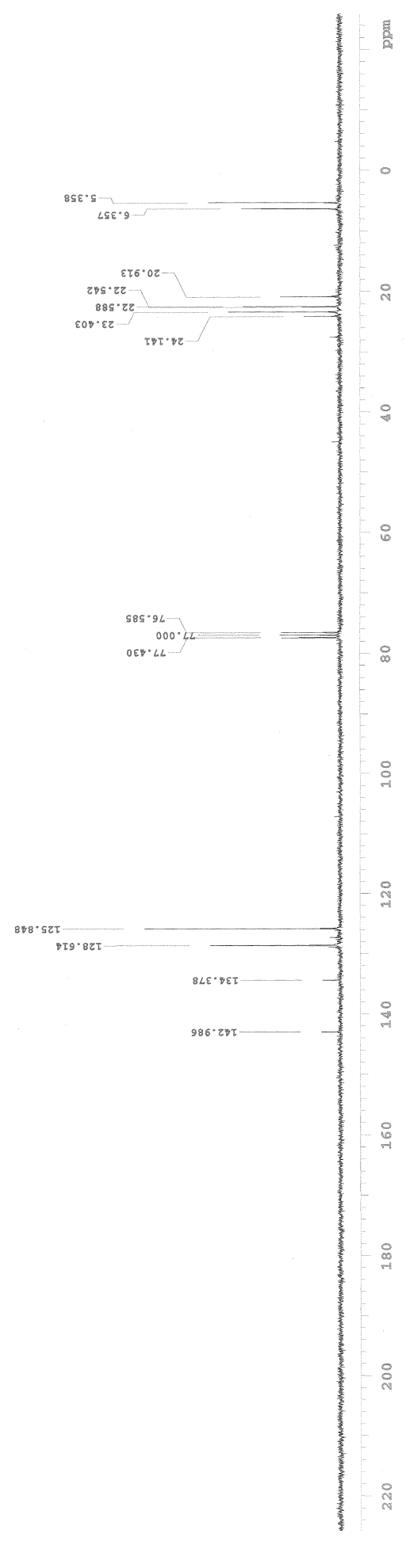



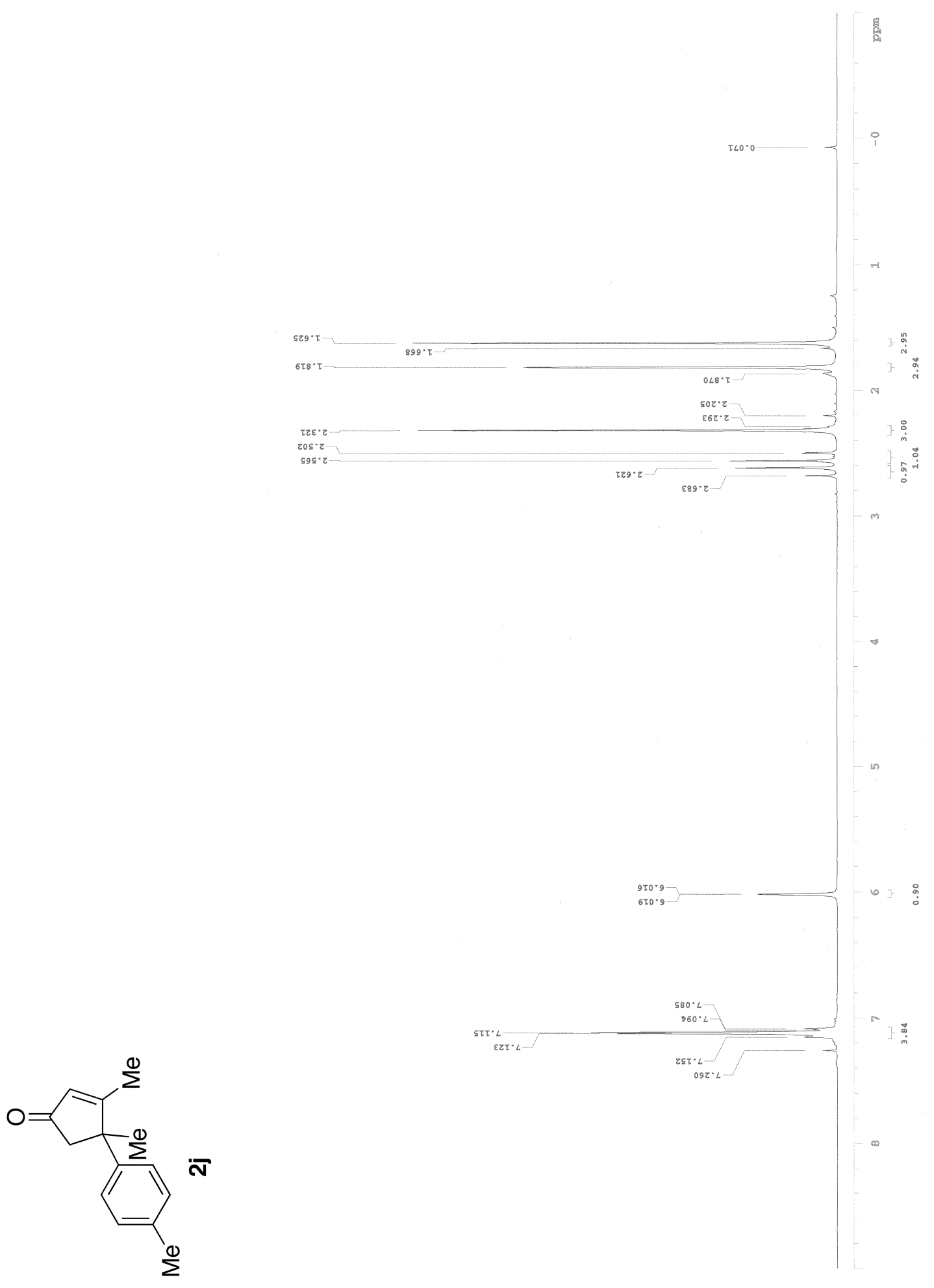


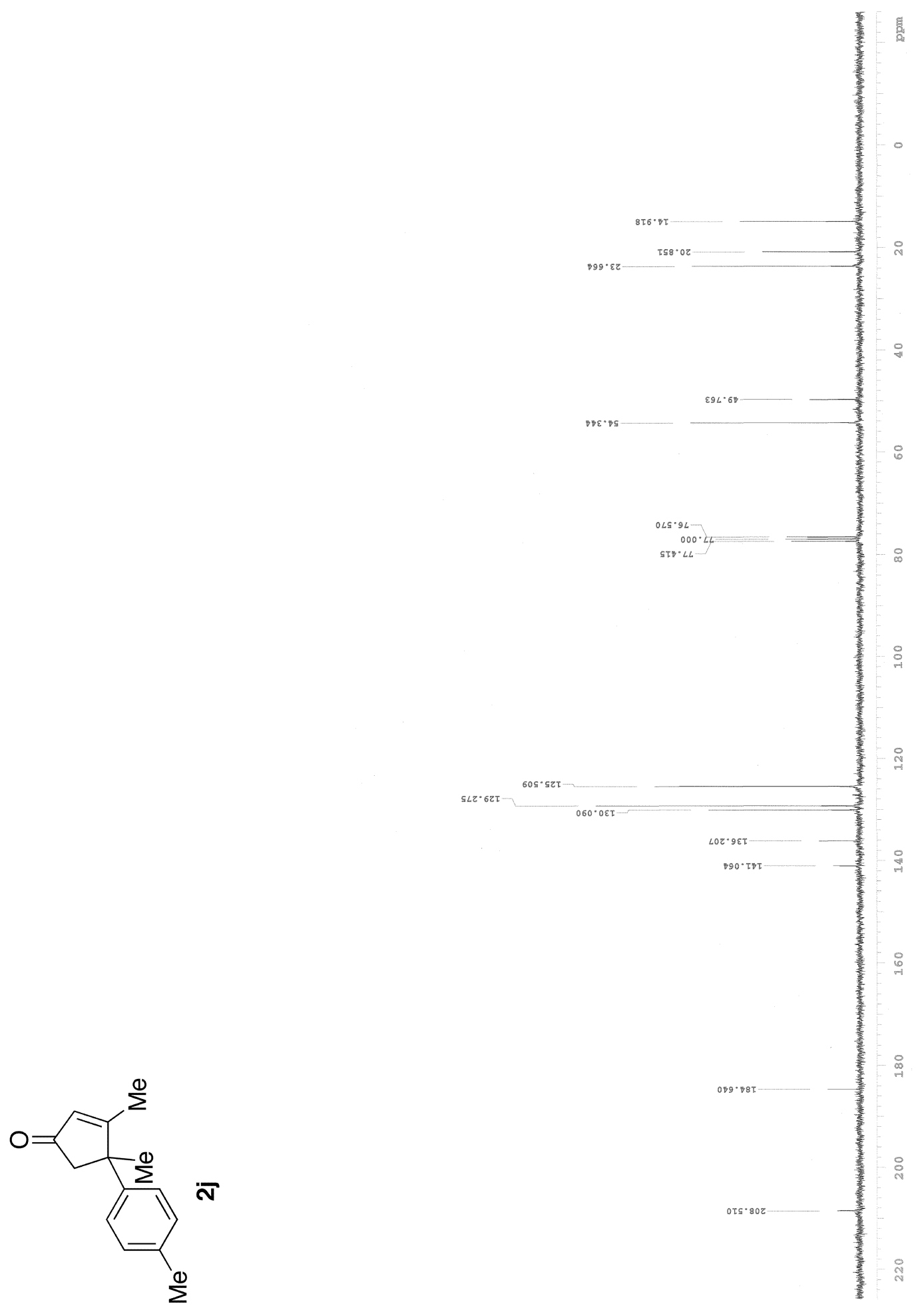



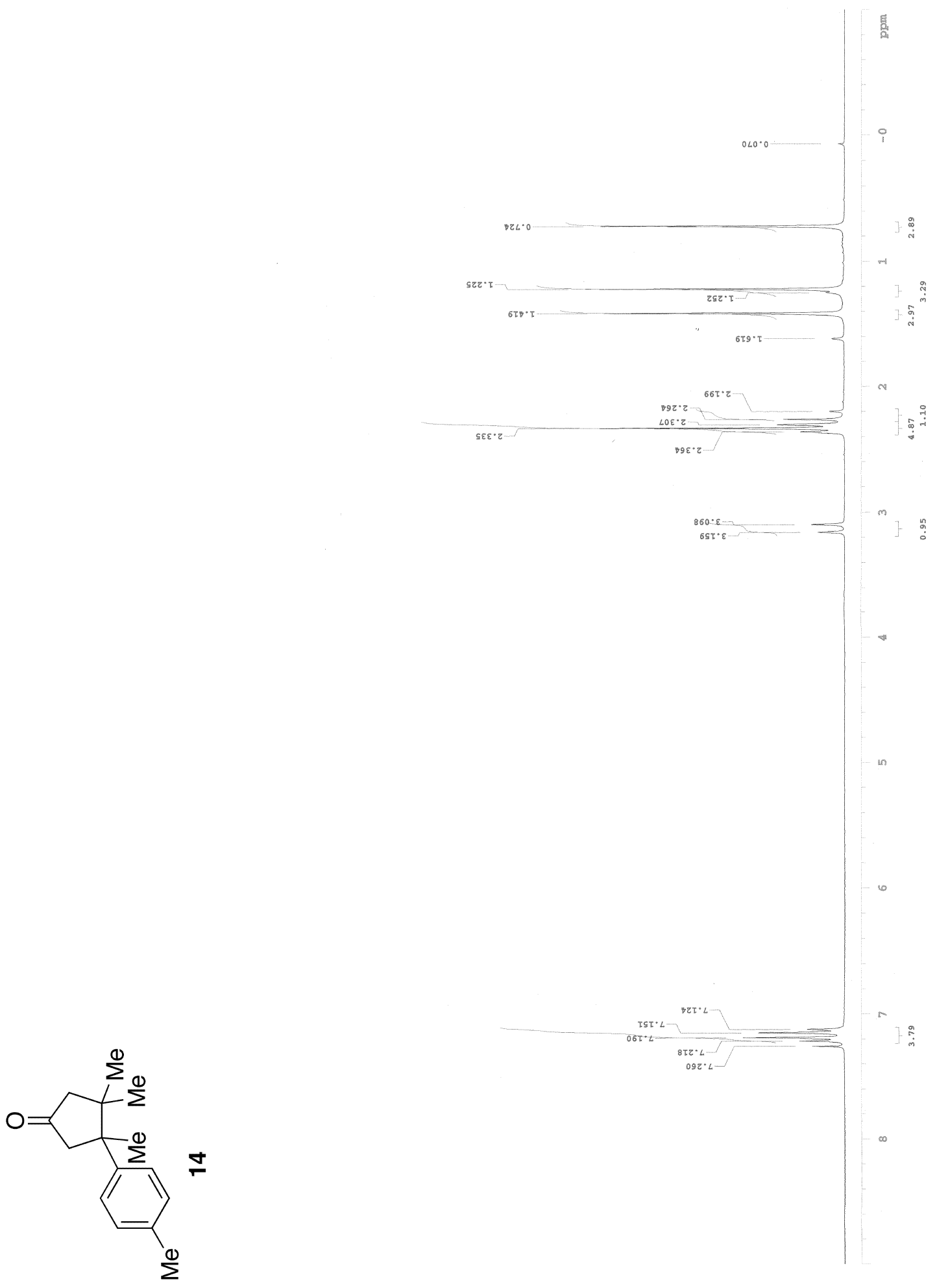


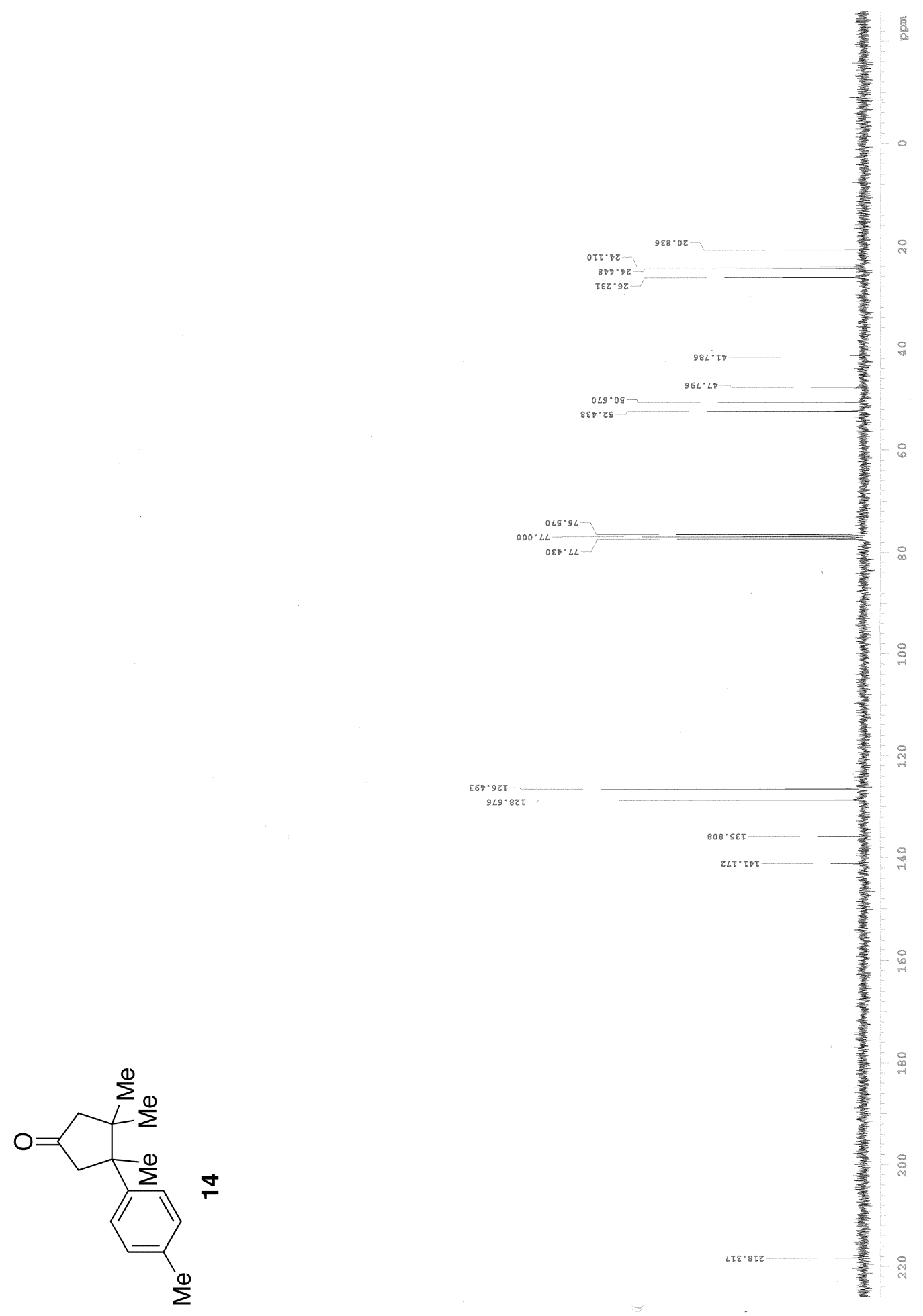

